\title{
THE PHENOMENOLOGY OF EXTENDED GAUGE AND HIGGS SECTORS AT THE LHC
}

\author{
By \\ Andrea Dawn Peterson \\ A dissertation submitted in partial fulfillment of \\ the requirements for the degree of \\ Doctor of Philosophy \\ (Physics) \\ at the \\ UNIVERSITY OF WISCONSIN-MADISON \\ 2014
}

Date of final oral examination: July 21, 2014

The dissertation is approved by the following members of the Final Oral Committee:

Vernon Barger, Professor, Physics,

Yang Bai, Assistant Professor, Physics,

Lisa Everett, Associate Professor, Physics,

Gabe Shaughnessy, Assistant Scientist, Physics,

Eric Bach, Professor, Computer Sciences 
In memory of my parents, Barbara and Richard.

They taught me to be curious, to be creative, to dream big dreams, to be strong and to never give up.

Their influence shows in everything I do. 


\title{
THE PHENOMENOLOGY OF EXTENDED GAUGE AND HIGGS SECTORS AT THE LHC
}

\author{
Andrea Dawn Peterson \\ Under the supervision of Professor Vernon Barger \\ At the University of Wisconsin-Madison
}

We consider prospects for detecting and measuring the properties of $Z^{\prime}, W^{\prime}$ and heavy Higgs bosons at the Large Hadron Collider (LHC). These particles are all well-motivated heavier counterparts to known SM particles. $Z^{\prime}$ and $W^{\prime}$ bosons arise when the SM gauge group is extended with additional $U(1)$ or $S U(2)$ factors. Heavy Higgs bosons are a feature of many models, including the Two Higgs Doublet Model (2HDM), supersymmetric (SUSY) models, and $W^{\prime}$ and $Z^{\prime}$ models.

First, we consider a number of common $Z^{\prime}$ models and present next-to-leading (NLO) and next-to-next-to-leading order (NNLO) predictions for the cross section, forwardbackward asymmetry, and rapidity distributions. We discuss methods for measuring the couplings of the $Z^{\prime}$ and distinguishing among models. $Z^{\prime}$ bosons with masses around $5 \mathrm{TeV}$ should be detectable at the LHC, and the couplings of a $2.5 \mathrm{TeV} Z^{\prime}$ could be measured within $0.1 \pi$ with a luminosity of $1 \mathrm{ab}^{-1}$. We also consider a hidden sector $Z^{\prime}$ that couples to standard model fermions via kinetic and mass mixing and serves as a mediator of isospin-violating interactions with dark matter. We combine the results of LHC $Z^{\prime}$ searches and dark matter direct detection experiments with global electroweak data to obtain mass-dependent constraints on the model parameters.

Next, we consider the fact that extra broken gauge symmetries are often accompanied by extended scalar sectors. If the masses of new Higgs particles are not too large, the $W^{\prime}$ bosons may decay into heavy Higgs particles, providing new possibilities for $W^{\prime}$ detection. We consider a simple scenario where the $W^{\prime}$ couplings to fermions are suppressed, making decays to scalar pairs the dominant decay mode. Potential final states include one or two gauge bosons plus missing energy. 
Finally, we turn our attention to Higgs pair production in the 2HDM. Higgs pair production is a valuable tool for measuring the triscalar couplings of the scalar potential. We consider both $h h$ resonant production and $h H$ associated production. We identify viable search channels for the LHC and provide their expected discovery sensitivities for a center-of-mass energy of $14 \mathrm{TeV}$ (LHC14) and a luminosity of $3 \mathrm{ab}^{-1}$. We find that discovery at the $95 \%$ C.L. is possible over most of the parameter space of the CP-conserving Type-II 2HDM. 


\section{ACKNOWLEDGMENTS}

First and foremost, I'd like to thank my advisor, Vernon Barger, for his unwavering support, thoughtful advice, and invaluable knowledge of physics. I learned so much from him, and would I not have had such a positive graduate school experience without

his understanding and encouragement. I'd also like to thank Lisa Everett for being a wonderful mentor and always having open and honest advice to share. I was fortunate to spend a fun and exciting year at Fermilab, and I'd like to thank the theory group there for their hospitality. In particular, I'd like to thank my advisor, Bogdan Dobrescu, for an interesting project and for helping me learn to be more confident as a physicist. Finally, I'd like to thank Gabe Shaughnessy, Yang Bai, Ran Lu, Jordi Salvado, and Danny Marfatia for interesting conversations and fruitful collaborations.

I made some great friends during grad school, in particular Will Parker, Kenny Rudinger, and roommates/fearsome lady physicists Bethany Reilly and Megan Wood. Thanks to them for fun adventures, conversations silly and serious, being my cooking experiment guinea pigs, and tolerating me when I get a little bit crazy. Above all, I'd like to thank my amazing husband Jim for being a true partner, supporting me through thick and thin, and inspiring me to be a better person. I couldn't do this without him. 


\section{TABLE OF CONTENTS}

LIST OF TABLES . . . . . . . . . . . . . . . . . . . . . . vii

LIST OF FIGURES . . . . . . . . . . . . . . . . . . . . . . . . . viii

$1 \quad$ Introduction . . . . . . . . . . . . . . . . . . . . . . . 1

1.1 The Standard Model . . . . . . . . . . . . . . . . . . . . . . . . . 2

1.2 Extended Gauge Symmetries . . . . . . . . . . . . . . . . . . . . . . . . . . . . . . . . . . .

1.3 Extended Higgs Sector . . . . . . . . . . . . . . . . . . . . . . . . 7

1.4 Experimental constraints . . . . . . . . . . . . . . . . 8

$2 \quad$ LHC and dark matter signals of $Z^{\prime}$ bosons _ . . . . . . . . . . . . . . . 11

2.1 Introduction . . . . . . . . . . . . . . . . . . . . . . 11

$2.2 \quad Z^{\prime}$ bosons at the LHC . . . . . . . . . . . . . . . . . . . . . . 12

2.3 Simulation . . . . . . . . . . . . . . . . . . . . . . . . . . . 15

2.4 Analysis . . . . . . . . . . . . . . . . . . . . . . . . . 19

$2.5 E_{6}$ Models . . . . . . . . . . . . . . . . . . . . . . . . . . 27

2.6 Dark Matter Interactions . . . . . . . . . . . . . . . . . . . . . . . . 29

2.7 Summary of $Z^{\prime}$ Analysis $\ldots \ldots \ldots$. . . . . . . . . . . . . . . . . . . . . . . . . . . . . . 39

$3 \quad W^{\prime}$ decays to odd Higgs particles . . . . . . . . . . . . . . . . . . 41

3.1 Introduction . . . . . . . . . . . . . . . . . . . . . 41

3.2 An $S U(2) \times S U(2) \times U(1)_{Y}$ model with odd Higgs sector . . . . . . . . . 42

3.2.1 Scalar spectrum . . . . . . . . . . . . . . . . 43

3.2 .2 Meta-sequential $W^{\prime}$ boson . . . . . . . . . . . . . . . . . . . . . 47

3.2.3 $Z^{\prime}$ mass and couplings . . . . . . . . . . . . . . . . . . . 50

$3.3 W^{\prime}$ and $Z^{\prime}$ decays . . . . . . . . . . . . . . . . . . . . . . . . 52

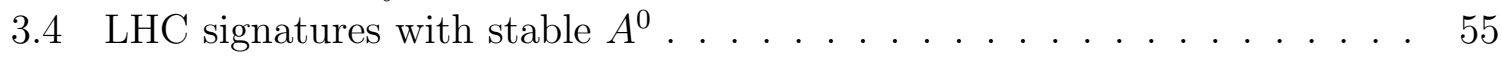

3.5 Summary of $W^{\prime}$ Analysis . . . . . . . . . . . . . . . . . . . . . . . . . . . 61

4 Measuring the 2HDM scalar potential . . . . . . . . . . . . . . . . . . . 63

4.1 Introduction $\ldots \ldots \ldots \ldots \ldots$

4.2 The Two Higgs Doublet Model . . . . . . . . . . . . . . . . . . . . . . . 65 
4.2.1 Yukawa couplings . . . . . . . . . . . . . . . . . . . . . 70

$4.2 .2 \quad$ Scalar couplings . . . . . . . . . . . . . . . . . . . . . . . 71

4.3 Heavy Higgs Branching Fractions . . . . . . . . . . . . . . . . . . . . . . 75

4.4 Higgs Pair Production Cross Section . . . . . . . . . . . . . . . . . . . . 77

4.5 Light Higgs pair production simulation . . . . . . . . . . . . . . . . . . . 81

4.5.1 The $h h \rightarrow b b \gamma \gamma$ channel . . . . . . . . . . . . . . . . . . 84

4.6 Associated $h H$ production . . . . . . . . . . . . . . . . . . . . . . 86

4.6 .1 The $h H \rightarrow b \bar{b} \gamma \gamma$ channel . . . . . . . . . . . . . . . . . . . . . . . 91

4.6 .2 The $h H \rightarrow 4 b$ channel . . . . . . . . . . . . . . . . . . . . . . . . 94

4.6 .3 The $h H \rightarrow Z Z b \bar{b}$ channel . . . . . . . . . . . . . . . . . . . . . . . 95

4.6 .4 The $h H \rightarrow h h h \rightarrow 4 b \gamma \gamma$ channel . . . . . . . . . . . . . . . . . . 98

4.6 .5 The $h H \rightarrow b \bar{b} t \bar{t}$ channel . . . . . . . . . . . . . . . . . . . . . . . . 99

4.7 Summary of 2HDM Analysis . . . . . . . . . . . . . . . . . . . . 100

LIST OF REFERENCES . . . . . . . . . . . . . . . . . . . . . . . . . 105 


\section{LIST OF TABLES}

Table Page

1.1 Gauge assignments for the SM fermions and Higgs doublet. The electric charge, $Q_{E M}$ is given by the sum of the $U(1)$ hypercharge, $Y$, and the weak isospin, $T_{L}^{3}$. $\ldots \ldots \ldots \ldots \ldots \ldots$

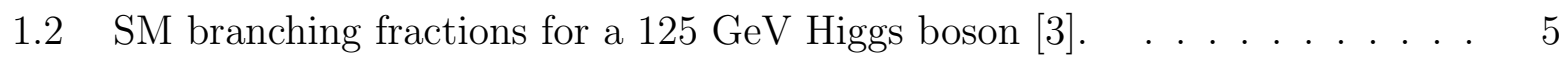

$2.1 \quad \mathrm{Z}^{\prime}$ gauge charges. For the LR model, $\alpha_{L R} \approx 1.58$. . . . . . . . . . . . . . . 18

$2.2 \quad Z^{\prime}$ decay widths and branching fractions, assuming no non-SM fermion decays. 19

$3.1 \quad$ Gauge assignments for the scalars $(\Delta$ and $\Phi)$ and SM fermions. . . . . . . . 43

$4.1 \quad$ Benchmark points of relevant couplings, production cross sections at LHC14 and branching fractions for the channels of interest. . . . . . . . . . . . . 103

4.2 The Higgs pair production processes that are sensitive to the couplings among the CP-even states. For each scalar coupling, the leading term in the expansion in the decoupling parameter, $c_{\beta-\alpha}=\cos (\beta-\alpha)$, is also shown. 103

4.3 The three benchmark points chosen to help elucidate the most viable channels as in Table 4.1, but with the expected statistical significance for LHC14 with $3 \mathrm{ab}^{-1}$ of integrated luminosity. . . . . . . . . . . . . . . . . . . . 104 


\section{LIST OF FIGURES}

Figure Page

1.1 Representative diagrams for Higgs production at the LHC, taken from [2]. . 6

1.2 Cross sections for production of a $125 \mathrm{GeV}$ Higgs in various channels at the LHC, taken from [2, 4]. We see that gluon fusion is the dominant production mechanism in a hadron collider. . . . . . . . . . . . . . . . . . . 6

1.3 CMS best fit values for the measured Higgs cross section relative to SM expectations in a number of production and decay modes, from [1].

$2.1 \quad$ Simulated NNLO dimuon invariant mass spectrum for a variety of $Z^{\prime}$ models with $M_{Z^{\prime}}=2.2 \mathrm{TeV}$ and $\sqrt{s}=8 \mathrm{TeV}$ (left) and $M_{Z^{\prime}}=2.5 \mathrm{TeV}$ and $\sqrt{s}=14 \mathrm{TeV}$ (right), both with a luminosity of $\mathcal{L}=100 \mathrm{fb}^{-1}$. $\ldots . . . .20$

2.2 NLO cross section for the process $Z^{\prime} \rightarrow l^{+} l^{-}$versus $\mathrm{Z}^{\prime}$ mass, integrated over the dilepton invariant mass peak region $( \pm 3 \Gamma)$ for $\sqrt{s}=8 \mathrm{TeV}$ (left) and $\sqrt{s}=14 \mathrm{TeV}$ (right). $\ldots \ldots \ldots \ldots . \ldots \ldots 21$

2.3 The parameter $p$ (as defined in Eq. 2.13) as a function of $r_{Z^{\prime}}=\frac{M_{Z^{\prime}}}{\sqrt{s}}$, along with an approximate tit to an arctangent function. Notice that simulated values (points) are shown for both $8 \mathrm{TeV}$ (squares) and $14 \mathrm{TeV}$ (circles) and agree well. . . . . . . . . . . . . . . . . . 22

$2.4 \quad$ FEWZ simulation (points) and fitted curve (lines) of the integrated $Z^{\prime}$ cross section for $\sqrt{s}=8 \mathrm{TeV}$ (left) and $\sqrt{s}=14 \mathrm{TeV}$ (right). We show only the $E_{6} \eta$, left-right, and sequential models for simplicity. . . . . . . . . . . . . . 23

2.5 Normalized distributions for $\frac{d \sigma}{d y}$ (left) and $\frac{d(F-B)}{d y}$ (right) for a $Z^{\prime}$ that couples to only up-type (solid) or down-type (dashed) quarks. We have set $M_{Z^{\prime}}=$ $2.5 \mathrm{TeV}$ at LHC14. Points are simulated data and lines are fitted curves. . . 24 
$2.6 \quad 95 \%$ C.L. regions for the couplings $c_{u}$ and $c_{d}$ for an average experiment (statistical errors only) for $\mathcal{L}=100 \mathrm{fb}^{-1}$ (left) and $\mathcal{L}=1 \mathrm{ab}^{-1}$ (right). The values of $c_{u}$ and $c_{d}$ for the $E_{6}$ family lie on the dashed red contour. Points are the theoretical values. . . . . . . . . . . . . . . 25

$2.795 \%$ confidence level regions for the couplings $e_{u}$ and $e_{d}$ for an average experiment for $\mathcal{L}=100 \mathrm{fb}^{-1}$ (left) and $\mathcal{L}=1 \mathrm{ab}^{-1}$ (right). Points are the theoretical values. . . . . . . . . . . . . . . . . . 26

2.8 Predicted on-peak forward-backward asymmetry versus mixing angle $\beta$ for the $E_{6}$ models (left) and $\alpha$ for the LR models (right), along with FEWZ simulated values and statistical errors for $M_{Z^{\prime}}=2.5 \mathrm{TeV}, \sqrt{s}=14 \mathrm{TeV}$, and $\mathcal{L}=100 \mathrm{fb}^{-1}$. . . . . . . . . . . . . . . . . 27

2.9 Simulated forward-backward asymmetry for a number of $Z^{\prime}$ models with $M_{Z^{\prime}}=2.5 \mathrm{TeV}$ and $\sqrt{s}=14 \mathrm{TeV}$. . . . . . . . . . . . . . . 28

2.10 Total width, normalized by the mass, and branching fractions to lepton, top, and neutrino pairs for the lower mass eigenstate $Z^{\prime}$ (left) and the higher mass eigenstate $Z^{\prime \prime}$ (right). For both, we include only decays into SM fermions..$~ 29$

2.11 Integrated peak cross section for the process $p p \rightarrow Z^{\prime} \rightarrow l^{+} l^{-}$as a function of mixing angle $\beta$, with $M_{Z^{\prime}}=2.5 \mathrm{TeV}$ at LHC14. . . . . . . . . . . . . . . 30

2.12 Integrated peak cross section for the process $p p \rightarrow Z^{\prime \prime} \rightarrow l^{+} l^{-}$at LHC14 versus mixing angle $\beta$. The mass of the heavier eigenstate is determined by the $Z^{\prime}$ mass and the mixing angle. . . . . . . . . . . . . . . . . . . . . . . . 31

2.13 Left: The 90\% C.L. allowed region for $S$ and $T$ based on the global data for $M_{H}=117 \mathrm{GeV}$ [57]. Right: This region translated into a 90\% CL region for $\epsilon$ and $\delta$ for $M_{Z^{\prime}}=1000 \mathrm{GeV}$. . . . . . . . . . . . . . . . 33

2.14 Contour plots of $f_{n} / f_{p}$ and $f_{p} / f_{n}$ as a function of $\epsilon$ and $\delta$. No limits are placed on the values of $\epsilon$ and $\delta . \ldots \ldots \ldots . \ldots . \ldots . . \ldots 34$

2.15 The 90\% C.L. upper bound on the spin independent dark matter-proton scattering cross section from XENON100 as a function of $f_{n} / f_{p}$. . . . . . . 35 
Figure

2.16 The cross section fro DM scattering on protons, $\sigma_{p}$ versus $Z^{\prime}$ mass for $f_{n} / f_{p}=$ 1. The thick red line at $\sigma_{p}=2.7 \times 10^{-45} \mathrm{~cm}^{2}$ is the XENON100 limit [27]; dots are the cross sections corresponding to pairs $(\epsilon, \delta)$ sampled uniformly over the allowed values. The dark matter mass and coupling to the $Z^{\prime}$ are set to $M_{\chi}=100 \mathrm{GeV}$ and $g_{\chi}=1$, respectively. . . . . . . . . . . . . . . 36

$2.17 \sigma_{p}$ versus $f_{n} / f_{p}$ mass for $M_{Z}^{\prime}=1000 \mathrm{GeV}$. The thick red line is the XENON100 upper bound; dots are the cross sections corresponding to pairs $(\epsilon, \delta)$ sampled uniformly through the allowed region shown in Fig 2.13 . The dark matter mass and coupling to the $Z^{\prime}$ are set to $M_{\chi}=100 \mathrm{GeV}$ and $g_{\chi}=1$, respectively. . . . . . . . . . . . . . . . . . . 37

$2.18 \sigma\left(p p \rightarrow Z^{\prime} \rightarrow l^{+} l^{-}\right)$versus $M_{Z}^{\prime}$. The thick red line is the most recent ATLAS limit [25]; dots are the cross sections corresponding to pairs $(\epsilon, \delta)$ sampled uniformly through the allowed region shown in Fig 2.13] . . . . . . . . . . . 38

2.19 Derived limits on $\epsilon$ and $\delta$ for $M_{Z^{\prime}}=500 \mathrm{GeV}$ (left) and $M_{Z^{\prime}}=1000 \mathrm{GeV}$ (right). The dotted blue lines represent the limit from global electroweak data (90\% C.L.), the solid red lines are the limit from XENON100 (90\% C.L.), and the dashed green lines are the limits from ATLAS at $\sqrt{s}=7 \mathrm{TeV}$ for $\mathcal{L} \approx 1 \mathrm{fb}^{-1}(95 \%$ C.L. $) . \ldots \ldots \ldots . \ldots . \ldots . \ldots 39$

$3.1 \quad W^{\prime}$ and $Z^{\prime}$ branching fractions as a function of mixing angle, for $M_{W^{\prime}}=3$ $\mathrm{TeV}, M_{H^{+}}=300 \mathrm{GeV}, M_{A}=200 \mathrm{GeV}$. . . . . . . . . . . . . 54

$3.2 \quad W^{\prime}$ production and cascade decays through odd Higgs particles. . . . . . . . 55

3.3 Leading-order cross sections times branching fractions for the processes $p p \rightarrow$ $W^{\prime} \rightarrow H^{+} A^{0}, H^{+} H^{0}$ and $p p \rightarrow Z^{\prime} \rightarrow H^{0} A^{0}, H^{+} H^{-}$at $\sqrt{s}=8 \mathrm{TeV}$ and 14 TeV. We have chosen $\tan \theta=1 / 4, M_{H^{+}}=300 \mathrm{GeV}$ and $M_{A}=200 \mathrm{GeV}$. . . 56

3.4 Transverse momentum distribution of the $W$ produced in $p p \rightarrow W^{\prime} \rightarrow$ $H^{+} A^{0} \rightarrow W A^{0} A^{0}$ when $M_{W^{\prime}}=2 \mathrm{TeV}$ (solid blue line) and $M_{W^{\prime}}=3 \mathrm{TeV}$ (dashed red line), for $\sqrt{s}=8 \mathrm{TeV}, M_{H^{+}}=300 \mathrm{GeV}$ and $M_{A}=200 \mathrm{GeV}$. For comparison, the $W p_{T}$ distribution (dotted black line) is included for $p p \rightarrow W \chi \bar{\chi}$ through a $\bar{q} \gamma_{\mu} q \bar{\chi} \gamma^{\mu} \chi$ contact interaction (for $\left.m_{\chi}=100 \mathrm{GeV}\right) . \quad . \quad 57$

$3.5 \quad E_{T}$ distribution (left) and transverse mass (right) distributions for $M_{H^{0}}=$ $300 \mathrm{GeV}$ and $M_{H^{0}}=1 \mathrm{TeV}$, all other parameters fixed $\left(M_{W^{\prime}}=3 \mathrm{TeV}\right.$, $\left.M_{A^{0}}=200 \mathrm{GeV}, \tan \theta=1 / 4\right) . \ldots \ldots \ldots \ldots \ldots$. . . . . . . . . . 59 
$3.6 \Delta \phi_{p_{T}^{\ell}, E_{T}}$ distribution, for $M_{H^{+}}=300 \mathrm{GeV}, M_{A}=200 \mathrm{GeV}$, and $\tan \theta=0.25$.

$3.7 \quad$ Exclusion limit in the $M_{W^{\prime}}-\tan \theta$ plane, derived from the CMS $W^{\prime} \rightarrow \ell \nu$ search [20], for $M_{H}=300 \mathrm{GeV}$ and $M_{A}=200 \mathrm{GeV}$. . . . . . . . . . . . . . 61

4.1 Representative Feynman diagrams which contribute to Higgs boson pair pro-

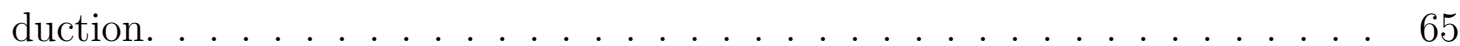

4.2 Regions that violate perturbative unitarity are shaded in the colors corresponding to the listed values of $\cos (\beta-\alpha)$. The decoupling of the heavy Higgs sector for $\cos (\beta-\alpha) \rightarrow 0$ can allow for a rather large value of $M . .7 .68$

4.3 Regions that violate perturbative unitarity (gray) and do not have a bounded potential (pink) are shaded for selected values of $\cos (\beta-\alpha)$ and $M / M_{H} . . \quad$. 69

4.4 Contours of the heavy Higgs Yukawa coupling to $t$ and $b$-quarks in the plane of $\tan \beta$ and $M_{H}$ for selected values of $\cos (\beta-\alpha)$. . . . . . . . . . . . 70

4.5 Contours of $\lambda^{h h H}$ in the plane of $\tan \beta$ and $M_{H}$ (in $\mathrm{GeV}$ ) for selected values of the decoupling parameter $\cos (\beta-\alpha)$. Included are the unitarity (gray) and vacuum stability (pink) constraints assuming $M=0.8 M_{H}$, the direct search exclusion limits (dashed pink) from CMS [21] and the $h h \rightarrow b b \gamma \gamma$ resonance search (purple) [149, 150, 151]. . . . . . . . . . . . . . . . . . . . 72

4.6 Contours of $B F(H \rightarrow h h)$ in the plane of $\tan \beta$ and $M_{H}$ for selected values of $\cos (\beta-\alpha)$. Additional experimental and theoretical constraints are shown as in Fig. 4.5. . . . . . . . . . . . . . . . . . . . . . . . . . . . . . . . 74

4.7 Contours of $B F(H \rightarrow b \bar{b})$ in the plane of $\tan \beta$ and $M_{H}$ for selected values of $\cos (\beta-\alpha)$. Additional experimental and theoretical constraints are shown

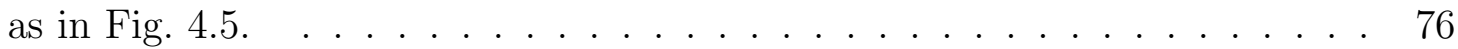

4.8 Contours of $B F(H \rightarrow t t)$ in the plane of $\tan \beta$ and $M_{H}$ for selected values of $\cos (\beta-\alpha)$. Additional experimental and theoretical constraints are shown

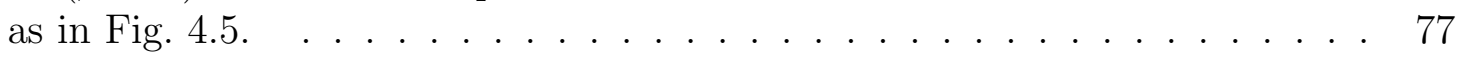

4.9 Contours of $B F\left(H \rightarrow W W^{(*)} / Z Z^{(*)}\right)$ in the plane of $\tan \beta$ and $M_{H}$ for selected values of $\cos (\beta-\alpha)$. Additional experimental and theoretical con-

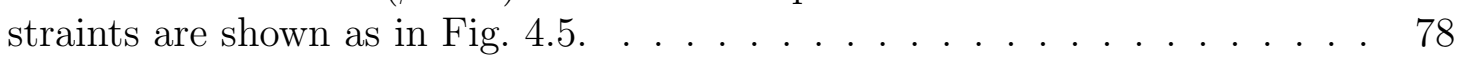


4.10 Contours of $\sigma(p p \rightarrow h h)$ in the plane of $\tan \beta$ and $M_{H}$ for selected values of $\cos (\beta-\alpha)$. Additional experimental and theoretical constraints are shown as in Fig. 4.5 . . . . . . . . . . . . . . . . . . . . . . 83

4.11 Contours of the luminosity required for $5 \sigma$ discovery in the plane of $\tan \beta$ and $M_{H}$ for selected values of $\cos (\beta-\alpha)$. Additional experimental and theoretical constraints are shown as in Fig. 4.5. . . . . . . . . . . . . . . . 87

4.12 Contours of the statistical significance with $3 \mathrm{ab}^{-1}$ of integrated luminosity in the plane of $\tan \beta$ and $M_{H}$ for selected values of $\cos (\beta-\alpha)$. Additional experimental and theoretical constraints are shown as in Fig. $4.5 \mid$. . . . . 88

4.13 Contours of $\sigma(p p \rightarrow h H)$ in the plane of $\tan \beta$ and $M_{H}$ for selected values of $\cos (\beta-\alpha)$. Additional experimental and theoretical constraints are shown

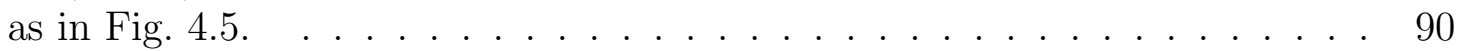

4.14 Contours of the statistical significance with $3 \mathrm{ab}^{-1}$ of integrated luminosity in the plane of $\tan \beta$ and $M_{H}$ for selected values of $\cos (\beta-\alpha)$. The bold dashed curves show the expected $5 \sigma$ significance, while the thin solid curves show the expected $95 \%$ C.L. reach. The colors correspond to different final states: $b \bar{b} \gamma \gamma$ (blue), $4 b$ (purple), $Z Z b \bar{b}$ (brown), $3 h$ (green), $t \bar{t} b \bar{b}$ in the single-lepton channel (red), and $t \bar{t} b b$ with two leptons (orange). Additional experimental and theoretical constraints are shown as in Fig. 4.5] . . . . . . . . . . . . . 92

4.15 The normalized $\Delta R$ distributions for the reconstructed light Higgs $b b$ pair (solid curves) and heavy Higgs $b b$ pair (dashed curves) are shown. The signal distributions for Benchmark $1\left(M_{H}=300 \mathrm{GeV}, \tan \beta=2\right.$, and $\cos (\beta-\alpha)=$ $0.1)$ are shown in red, while the background is shown in blue. The signal events tend towards smaller values of $\Delta R$, indicating that the Higgs bosons (especially the $h$ ) are boosted. . . . . . . . . . . . . . . . 96

4.16 The invariant mass of the heavier $b b$ pair in the $4 b$ channel after the cuts on $M_{h}$ and $\Delta R$ is shown. Signal (red) is shown for Benchmark $1\left(M_{H}=300\right.$ $\mathrm{GeV}, \tan \beta=2$, and $\cos (\beta-\alpha)=0.1$ ). The background distribution (blue) drops off quickly with $M(b b)$, which leaves the resonance at $M_{H}$ clearly discernible. . . . . . . . . . . . . . . . . . 97 
4.17 The transverse mass of the top quark pair in the $t \bar{t} b \bar{b}$ with one lepton channel. The signal (red) is shown for Benchmark $3(\cos (\beta-\alpha)=0.02, \tan \beta=1$, and $M_{H}=500 \mathrm{GeV}$ ). The signal drops off sharply above $M_{H}$, while the background (blue) decreases more gradually. . . . . . . . . . . . . . . . 100 


\section{Chapter 1}

\section{Introduction}

The Standard Model (SM) of particle physics has been remarkably successful, withstanding ever-more rigorous tests over the course of several decades. In July 2012, the ATLAS and CMS collaborations at the Large Hadron Collider (LHC) announced the discovery of the final piece of the SM puzzle: a Higgs boson with a mass of $125 \mathrm{GeV}$. This amazing discovery validates the fundamental theoretical tenet that the electroweak gauge symmetry is spontaneously broken. So far, LHC measurements of the Higgs couplings to other particles are all consistent with their SM-predicted values [1]. This is satisfying, to a degree, but it is also mystifying that the SM should work quite so well, when we know it faces several fundamental challenges. For one, the Higgs mass, measured to be $125 \mathrm{GeV}$, is not predicted in the SM. In fact, it requires theoretically unappealing fine-tuning of model parameters, since otherwise quantum effects should drive the Higgs mass to very large values near the Planck scale, where gravity becomes important. The Planck scale is many, many orders of magnitude larger than the electroweak scale, a fact the SM cannot explain.

Another major issue is that the SM provides no dark matter candidate. Dark matter interacts gravitationally but has no electric charge, so we cannot observe it through its interactions with photons, as with ordinary matter. Copious astronomical observations, such as measurements of galactic rotation rates and gravitational lensing effects, show that about $85 \%$ of the matter in our universe is dark. Observations from colliding 
clusters of galaxies, like the famous bullet cluster, show that dark matter has very weak self-interactions, while analyses of the structure formation indicate it is mostly cold (nonrelativistic). Many experiments are underway to detect dark matter directly, including LUX, XENON, CDMS, and CRESST. A few potential signals have been detected, but thus far there has been nothing definitive, and some of the results are in tension with one another [2].

Many expect that $\mathrm{TeV}$-scale new physics beyond the Standard Model (BSM) will resolve these issues. The LHC experiments at 7 and $8 \mathrm{TeV}$ center-of-mass energy have not found evidence for significant deviations from the SM or any new particles, however the LHC upgrade to $14 \mathrm{TeV}$, with 10 times the present luminosity, may change this. In this thesis, we focus on two simple possibilities for new physics at the LHC: extending the electroweak gauge symmetry, or expanding the scalar symmetry breaking sector. While often considered in separate contexts, these two ideas are closely related, as we will point out in Chapter 3. Both scenarios lead to heavier counterparts of known SM particles, which could potentially be detected at the LHC. We will discuss direct and indirect methods of detecting and measuring the properties of these new particles, and how to connect these observations to an underlying physics model.

\subsection{The Standard Model}

The Standard Model is a theory of subatomic particles and the forces and symmetries that govern their interactions. It describes three of the four fundamental forces (the strong and weak nuclear forces and electromagnetism, but not gravity) in terms of a local gauge symmetry $S U(3)_{C} \times S U(2)_{L} \times U(1)_{Y} . S U(3)_{C}$ corresponds to the strong interactions, while the weak and electromagnetic interactions are unified into the electroweak gauge group $S U(2)_{L} \times U(1)_{Y}$, which is broken to $U(1)_{E M}$ by the Higgs mechanism. How

gravity can be incorporated into this picture is an area of active research. Each of these symmetry groups is associated with a coupling constant that determines the strength of the force. Due to quantum effects, the strengths of these couplings depend on the 
energy scale of the process under consideration. The $S U(3)_{C}$ coupling is largest at low energies or, equivalently, long distances, while the the strength of the electromagnetic and weak forces is quite small at long distances.

The SM contains 12 types of fermions (spin-1/2 particles): six quarks (up, down, charm, strange, top and bottom) and six leptons (electrons, muons, and taus, and their corresponding neutrinos), plus an oppositely-charged antiparticle for each of these. These particles transform under representations of the SM gauge group. Only quarks interact via the strong force; they form an $S U(3)_{C}$ triplet, carrying one of three charges known as colors. Since the strength of the strong force increases at low energies, free quarks are not observed. Instead, they are found in color-neutral bounds states called hadrons, which consist of either three quarks or a quark-antiquark pair. The most common hadrons are the protons and neutrons that make up atoms.

Left-handed quarks and leptons are doublets under $S U(2)_{L}$ :

$$
L_{L}=\left(\begin{array}{c}
\nu_{i} \\
\ell_{i}
\end{array}\right)_{L}, \quad Q_{L}=\left(\begin{array}{c}
u_{i} \\
d_{i}
\end{array}\right)_{L},
$$

where $i$ is a generation index such that $\nu_{i}=\nu_{e}, \nu_{\mu}, \nu_{\tau} ; \ell=e, \mu, \tau ; u_{i}=u, c, t$; and $d_{i}=d, s, b$. The corresponding right-handed fields

$$
u_{R}, \quad d_{R}, \quad \ell_{R}
$$

are singlets under $S U(2)_{L}$. The gauge assignments of the SM fermions are given in Table 1.1. The SM does not explicitly include right-handed neutrinos. However, so-called sterile (non-interacting) neutrinos can be added easily, since right-handed neutrinos would be singlets under the SM gauge group. It is usually assumed that right-handed neutrinos are very heavy, so the minuscule masses of the left-handed neutrinos can be accounted for with the see-saw mechanism.

Each subgroup of the SM is associated with force-carrying gauge bosons. The exchange of these particles mediates force interactions between matter particles. There are eight QCD gauge bosons, called gluons, transforming in the adjoint representation 


\begin{tabular}{|c||c|c|c|c|}
\hline & $S U(3)_{C}$ & $S U(2)_{L}$ & $U(1)_{Y}$ & $Q_{E M}=T_{L}^{3}+Y$ \\
\hline \hline$Q_{L}$ & 3 & 2 & $+\frac{1}{6}$ & $+\frac{2}{3},-\frac{1}{3}$ \\
\hline$L_{L}$ & 1 & 2 & $-\frac{1}{2}$ & $0,-1$ \\
\hline$u_{R}, d_{R}$ & 3 & 1 & $+\frac{2}{3},-\frac{1}{3}$ & $+\frac{2}{3},-\frac{1}{3}$ \\
\hline$e_{R}$ & 1 & 1 & +1 & -1 \\
\hline$\Phi$ & 1 & 2 & $+\frac{1}{2}$ & 0 \\
\hline \hline
\end{tabular}

Table 1.1 Gauge assignments for the SM fermions and Higgs doublet. The electric charge, $Q_{E M}$ is given by the sum of the $U(1)$ hypercharge, $Y$, and the weak isospin, $T_{L}^{3}$.

of $S U(3)$. There are three weak gauge bosons, $W^{ \pm}$and $W^{0} . W^{0}$ mixes with the $U(1)_{Y}$ gauge boson $B$, leading to two physically-observed mass eigenstates: the massive $Z$ boson and the massless photon, $A$. Explicit mass terms for gauge bosons are not allowed by gauge invariance; instead, the masses are generated through the Higgs mechanism. If the scalar potential,

$$
V=\frac{m^{2}}{2} \Phi^{\dagger} \Phi+\frac{\lambda}{4}\left(\Phi^{\dagger} \Phi\right)^{2}
$$

has a stable minimum away from $\Phi=0$, then we can shift the Higgs field by its vacuum expectation value $(\mathrm{VEV}), v \approx 246 \mathrm{GeV}$. Then the scalar field can be rewritten as

$$
\Phi=\left(\begin{array}{c}
G^{+} \\
\frac{\left(h+v+i G^{0}\right)}{\sqrt{2}}
\end{array}\right) .
$$

After symmetry breaking, the expansion of the Higgs kinetic terms about $v$ generates mass terms for the weak bosons. With an appropriate choice of gauge, the Goldstone bosons, $G^{0}$ and $G^{ \pm}$, are "eaten" and become the longitudinal degrees of freedom for the now-massive $W$ and $Z$, leaving one physical Higgs particle, $h$. The Higgs mechanism can also generate fermion masses through Yukawa couplings of the form

$$
y_{d} \bar{q}_{L} \Phi d_{R}, \quad y_{u} \bar{q}_{L} \tilde{\Phi} u_{R}, \quad y_{\ell} \bar{\ell}_{L} \Phi e_{R}
$$


The Higgs boson, $h$, has direct couplings to $W$ and $Z$ bosons and fermions, and gains couplings to photons and gluons through loops. The fermion couplings are proportional to their masses (since both come from the Yukawa terms), so the Higgs interacts most strongly with top quarks. It also has sizable couplings to $W$ and $Z$ bosons. The SM Higgs boson, with a mass of $125 \mathrm{GeV}$, is too light to decay to tops or on-shell weak bosons, so its primary decay is to $b$ quarks. The SM Higgs branching fractions for the most important channels are shown in Table 1.2. The Higgs can be produced through several processes at the LHC: gluon fusion, vector boson fusion, associated production with a vector boson, or associated production with a top quark pair. Diagrams for these processes are shown in Figure 1.1, and their cross sections as a function of collider energy are shown in Figure 1.2 .

\begin{tabular}{|c|c|}
\hline Decay channel & Branching Fraction \\
\hline \hline$H \rightarrow b \bar{b}$ & $57.7 \%$ \\
$H \rightarrow W W$ & $21.5 \%$ \\
$H \rightarrow \tau^{+} \tau^{-}$ & $6.3 \%$ \\
$H \rightarrow Z Z$ & $2.6 \%$ \\
$H \rightarrow \gamma \gamma$ & $0.2 \%$ \\
\hline
\end{tabular}

Table 1.2 SM branching fractions for a $125 \mathrm{GeV}$ Higgs boson [3].

\subsection{Extended Gauge Symmetries}

One simple and well-motivated extension of the SM is to add an extra $U(1)^{\prime}$ factor to the gauge group and its associated gauge boson, the $Z^{\prime}$. If $U(1)^{\prime}$ is broken at the $\mathrm{TeV}$ scale, the $Z^{\prime}$ could be observed as a heavy dilepton or dijet resonance at the LHC. Grand unified theories (GUTs), embed the SM gauge group in a larger symmetry group, are a strong motivation for $Z^{\prime}$ searches. Grand Unified theories attempt to explain a number of theoretical issues, including why charge is quantized, why the SM is chiral (asymmetric 


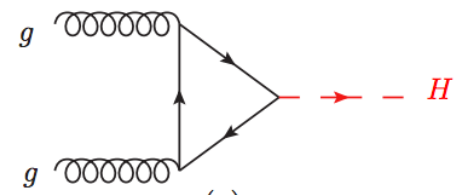

(a)

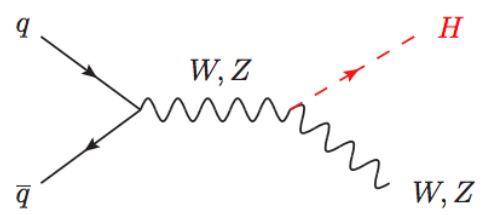

$(c)$

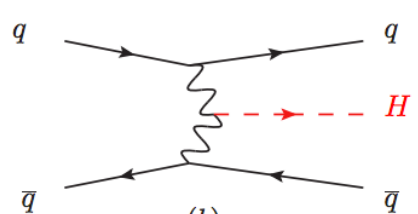

(b)

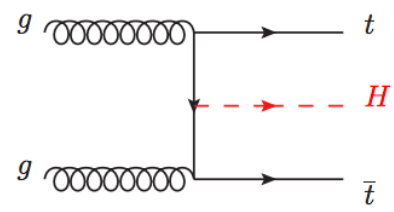

$(d)$

Figure 1.1 Representative diagrams for Higgs production at the LHC, taken from [2].

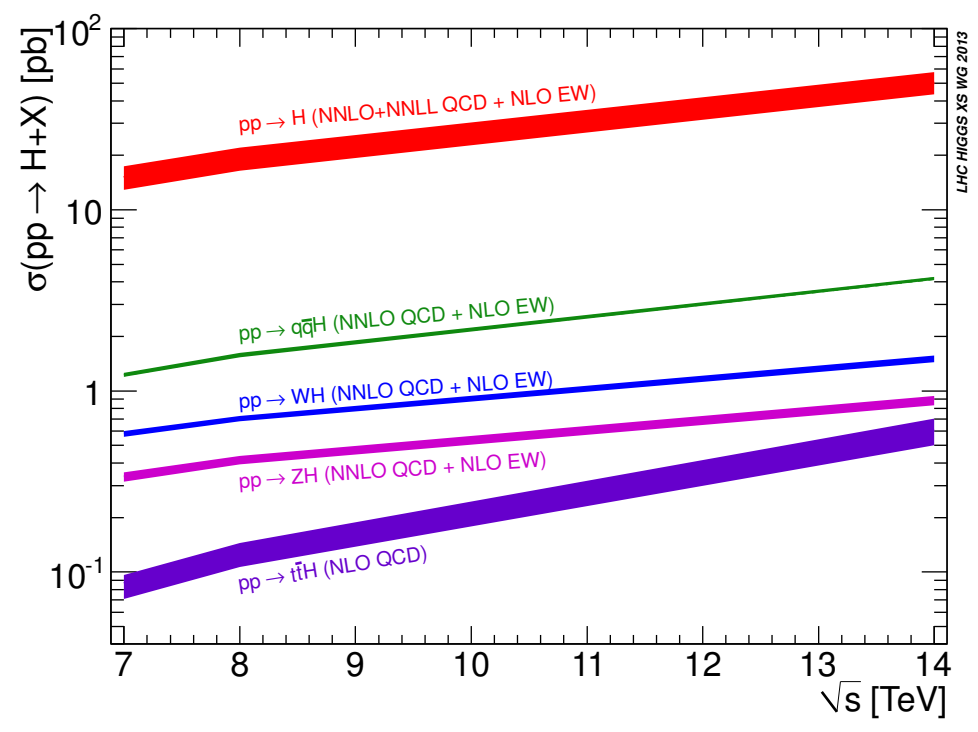

Figure 1.2 Cross sections for production of a $125 \mathrm{GeV}$ Higgs in various channels at the LHC, taken from [2, 4]. We see that gluon fusion is the dominant production mechanism in a hadron collider.

between right- and left-handed particles), and why there are three generations of matter. They also provide for gauge coupling unification, something that is hinted at, but does not quite work, in the SM [2]. There are several common types of GUTs that lead to $Z^{\prime}$ bosons. One class is the left-right symmetric models that contain a $U(1)$ for $B-L$ (baryon number - lepton number), which can be further embedded in $S O(10)$. Another 
is the string theory-motivated $E_{6}$ GUT, which breaks down to include multiple $U(1)$ factors [5, 6, 7, 8, 9]. Z' $Z^{\prime}$ bosons also arise in Little Higgs models and models with extra dimensions [5]. An area of considerable recent interest has been dark sector $U(1)$ symmetries, where dark matter is charge under the $U(1)$, but SM fermions are not. This leads to dark photon or $Z^{\prime}$-mediated dark matter interactions [10, 11, 12].

We can also consider adding larger gauge groups to the $\mathrm{SM}$, such as $S U(N)$ factors, where $N \geq 2$. One famous example is the left-right symmetric model [13, 14, 15, 16, 17,. Since the left-handed fermions are charged under $S U(2)_{L}$, it would seem natural to add a similar group $S U(2)_{R}$ under which the right handed fermions transform as doublets. This is implemented by assuming a $S U(2)_{L} \times S U(2)_{R} \times U(1)_{B-L}$ symmetry at high scales, with $S U(2)_{R} \times U(1)_{B-L}$ breaking to $U(1)_{Y}$ at the $\mathrm{TeV}$ scale (or higher). Despite the popularity of this model, it is not the only model based on a $S U(2) \times S U(2) \times U(1)$ gauge structure. Other options include models where quarks and leptons are charged under different $S U(2)$ s, or where the third generation is assigned to its own $S U(2)$ [16]. It is also possible that none of the SM fermions are charged under the second $S U(2)$, so that the $W^{\prime}$ acquires left-handed couplings to SM fermions only through mixing with the $W$. As in the case of the $U(1)$-extended symmetry, if the symmetry breaking scale is not too high, the heavy $W^{\prime}$ and $Z^{\prime}$ bosons associated with the new $S U(2)$ could be detectable at the LHC.

\subsection{Extended Higgs Sector}

The SM Higgs mechanism requires only one complex doublet scalar to generate masses for the $W$ and $Z$ bosons and the fermions. However, it is often the case in BSM scenarios that more than one Higgs multiplet is allowed, or even required. The most common option is to add extra $S U(2)$ doublets or singlets. Higher $S U(2)$ representations, for example triplets, are disfavored due their effect on the ratio of the $W$ and $Z$ masses, a very precisely measured quantity. The most widely-studied model is the Two Higgs Doublet Model (2HDM), in which a second complex doublet is added to the scalar 
sector, adding heavy Higgs states to the physical spectrum [18]. In the CP-conserving case, there are two charged Higgs $H^{ \pm}$, a neutral CP-even Higgs $H^{0}$, and a neutral CPodd Higgs $A^{0}$. There are several incarnations of the 2HDM, differing in how the Higgs particles interact with fermions. A particularly interesting case is the Type-II 2HDM, which corresponds to the Higgs sector of the Minimal Supersymmetric Standard Model (MSSM).

Extra Higgs particles often show up in models with extended gauge symmetries as well, as they are required to break the new symmetry groups to the SM gauge group. $W^{\prime}$ models usually require two scalar multiplets, often a combination of $S U(2)$ triplets, doublets, or bidoublets (i.e. a scalar that transforms in the fundamental representation of both factors of $S U(2))$. The particular choice of Higgs representation, as well as the hierarchy of the symmetry-breaking scales, has a significant impact on the spectrum and couplings of the scalar and vector bosons.

In any of these models, measuring the properties of the Higgs boson will be central to the search for new physics. Small deviations from SM predictions of Higgs couplings and decays could hint at new physics long before a new particle is directly detected. While we have begun to measure the couplings of the Higgs to weak bosons, photons, gluons and fermions, the Higgs potential itself has not yet been subject to experimental scrutiny. Such analysis requires the more challenging measurements of triple and quartic Higgs self-interactions via pair production of Higgs bosons. However, this important avenue of pursuit should soon be possible with data from the upcoming LHC14 run, as will increasingly precise measurements of the Higgs fermion and gauge boson couplings. Current measurements from CMS of the Higgs cross section for different production and decay modes are shown in Figure 1.3.

\subsection{Experimental constraints}

All of these new particles are being actively searched for at the LHC. So far, only null results have been found, leading to lower bounds on the masses of the new particles. 


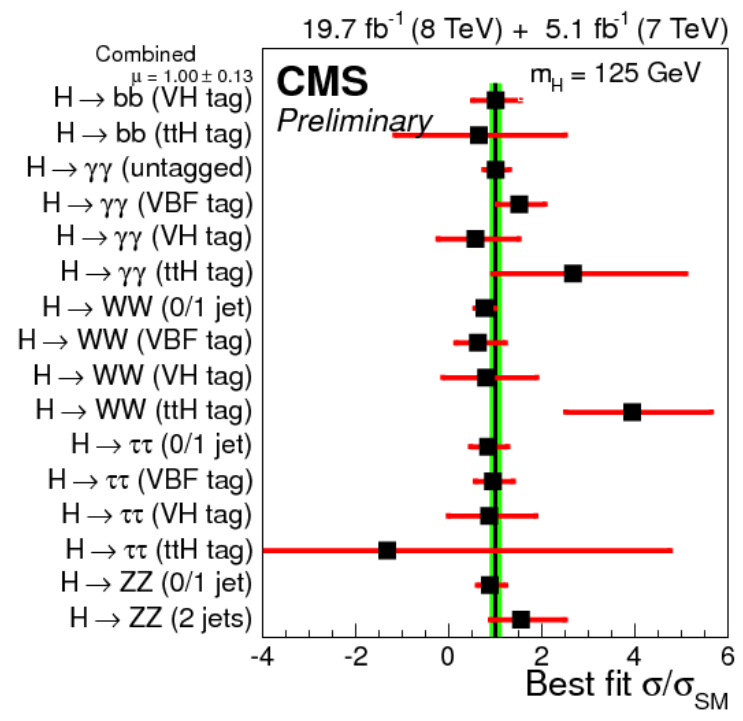

Figure 1.3 CMS best fit values for the measured Higgs cross section relative to SM expectations in a number of production and decay modes, from [1].

With $20 \mathrm{fb}^{-1}$, CMS excludes $Z^{\prime}$ masses much below 2500-3000 GeV [19]. $W^{\prime}$ bosons are excluded below 3.35 TeV [20]. SM-like heavy Higgs bosons are excluded for masses in the range of 127-710 GeV [21]. However, these bounds are highly model-dependent. Simple adjustments to the standard assumptions can dramatically change the picture, allowing "excluded" models to remain promising. This is a point we will return to frequently in this work.

New physics models are also constrained by the extremely precise measurements of the $Z$ boson couplings at the $Z$-pole [2]. The ongoing measurements of the Higgs mass and couplings provide additional constraints. These constraints are especially important for the models considered in this thesis, as the new gauge bosons and Higgs scalars generally mix with their SM counterparts, altering their properties. This leaves two options: either the mixing angles between sectors are small, or the mass scales of the SM and new physics are quite separated.

This thesis is organized as follows: In Chapter 2, we discuss the detecting and measuring the couplings of $Z^{\prime}$ bosons at the LHC. For a spectrum of benchmark models, we 
discuss how the rapidity and forward-backward distributions can be used to determine the couplings of the $Z^{\prime}$ and differentiate between models. We also consider a dark-sector $Z^{\prime}$ that gains couplings to the SM through kinetic and mass mixing with the SM $Z$, and obtain constraints from dark matter direct detection experiments. In Chapter 3, we consider a left-handed $W^{\prime}$ models with fermion couplings proportional to the SM $W$ couplings. We use this model to demonstrate that $W^{\prime}$ bosons can decay dominantly into scalar pairs, and discuss collider signatures of such decays. Finally, in Chapter 4, we consider production of Higgs pairs $h h$ and $h H$ in the Type-II CP-conserving 2HDM. These processes are valuable for measuring the triscalar terms of the Higgs potential. We identify viable search channels and evaluate the LHC discovery reach for each process. We also discuss the use of Multivariate Analysis (MVA) techniques to improve LHC sensitivity. 


\section{Chapter 2}

\section{LHC and dark matter signals of $Z^{\prime}$ bosons}

\subsection{Introduction}

A simple extension of the Standard Model (SM) is the addition of an extra $U(1)$ gauge symmetry, with associated neutral $Z^{\prime}$ gauge boson. Extra $U(1)$ symmetries are a necessary part of many interesting new physics scenarios, including several Grand Unified Theories and string-inspired model constructions. Generic $Z^{\prime}$ models can have many new physics features, including generation-dependent couplings, $Z-Z^{\prime}$ mixing, and new fermions; see e.g., Refs. [5, 6, 7, 8, 9]. We first study the simplest models with generation-independent couplings and no $Z-Z^{\prime}$ mixing. There are a number of such models that are theoretically relevant, such as the $E_{6}$ GUT models and the $B-L$ model. In Section 2.6, we consider a model that includes mass and kinetic mixing between the $Z^{\prime}$ and the $Z$ [10], which has applications to isospin-violating dark matter scattering [11].

We expand the simulation code FEWZ 2.1 (Fully Exclusive $W$ and $Z$ Production) [22, 23] to study the production and decay of $Z^{\prime}$ bosons at the LHC through the process $p p \rightarrow Z^{\prime} \rightarrow l^{+} l^{-}$. FEWZ includes up to NNLO in perturbative QCD and is fully differential in the lepton phase space. This allows for the precise calculation of the $Z^{\prime}$ cross sections and differential distributions with realistic experimental acceptances. In Sections 2.2 and 2.3 , we briefly review $Z^{\prime}$ detection and introduce several common benchmark models that we use to demonstrate the efficacy and validity of our simulation.

In Section 2.4 we use FEWZ to derive a semi-empirical expression for the differential

cross section $\frac{d \sigma}{d y d \cos \theta}$. We show that, with sufficient data, this formula can be used 
to determine the couplings of the $Z^{\prime}$ and set limits on model parameters with good precision.

In Section 2.5, we apply our fit to the $E_{6}$ model class and the production of two $Z^{\prime}$ bosons. The heavier of the two mass eigenstates is often assumed to be too heavy for collider detection, but we show that it could be accessible at the LHC for a certain range of mixing angles. Throughout, we focus on $Z^{\prime}$ masses of a few $\mathrm{TeV}$, as the LHC lower limits with approximately $5 \mathrm{fb}^{-1}$ of data fall in the vicinity of $2-2.5 \mathrm{TeV}$ for the considered models [24].

In Section 2.6 we study the phenomenology of a $Z^{\prime}$ scenario in which SM particles are uncharged under the new $U(1)^{\prime}$. In this case, SM particles interact with a new sector through kinetic and mass mixing of the $Z^{\prime}$ with the $Z$. Such a $Z^{\prime}$ could act as a dark matter mediator, with isospin-violating dark matter scattering arising naturally. Then, there are two complementary ways to test such a model: the production of $Z^{\prime}$ resonances in collider experiments, and the direct detection of dark matter particles. We combine the data from LHC $Z^{\prime}$ searches [25, 26] and the XENON100 dark matter experiment [27] with global electroweak data to constrain the kinetic and mass mixing angles. We find that the electroweak, collider, and dark matter data provide comparable limits.

\section{$2.2 Z^{\prime}$ bosons at the $\mathrm{LHC}$}

If the $Z^{\prime}$ couples to standard model quarks and leptons, it could be detected at the LHC as a resonance in the dilepton channel through the experimentally well-studied process $p p \rightarrow Z^{\prime} \rightarrow l^{+} l^{-}[28$, 29, 30, 31, 32, 33]. We focus on the dielectron and dilmuon channels, though decays to $\tau$-lepton pairs can also be useful [34]. The SM background to this process is fairly small, consisting mostly of Drell-Yan (DY) $Z / \gamma^{*}$ events and a smaller number of $t \bar{t}$ and multijet events [25, 26].

The differential cross section for the DY process is 6]

$$
\frac{d \sigma}{d Q^{2}}=\frac{1}{s} \sigma\left(Z^{\prime} \rightarrow l^{+} l^{-}\right) W_{Z^{\prime}}\left(s, Q^{2}\right)+\text { interference terms }
$$


where $\sqrt{s}$ is the collider energy and $Q$ is the center-of-mass energy of the dilepton pair. The first term on the right hand side is the pure $Z^{\prime}$ contribution, factored into two parts: a hadronic structure function containing the QCD dependence, $W_{Z^{\prime}}\left(s, Q^{2}\right)$, and the partonic cross section,

$$
\sigma\left(Z^{\prime} \rightarrow l^{+} l^{-}\right)=\frac{1}{4 \pi} \frac{g_{l_{L}}^{2}+g_{l_{R}}^{2}}{288} \frac{Q^{2}}{\left(Q^{2}-M_{Z^{\prime}}^{2}\right)^{2}+M_{Z^{\prime}}^{2} \Gamma_{Z^{\prime}}^{2}} .
$$

The interference terms read

$\sigma\left(Z^{\prime}, X\right)=\frac{g_{Z^{\prime}} g_{X}}{2 \pi}\left(\frac{z_{l_{L}} z_{l_{L}}^{X}+z_{l_{R}} z_{l_{R}}^{X}}{288}\right) \frac{\left(Q^{2}-M_{Z^{\prime}}^{2}\right)\left(Q^{2}-M_{X}^{2}\right)+M_{Z^{\prime}} M_{X} \Gamma_{Z^{\prime}} \Gamma_{X}}{\left[\left(Q^{2}-M_{Z^{\prime}}^{2}\right)^{2}+M_{Z^{\prime}}^{2} \Gamma_{Z^{\prime}}^{2}\right]\left[\left(Q^{2}-M_{X}^{2}\right)^{2}+M_{X} \Gamma_{X}^{2}\right]}$,

where $X=\gamma, Z$. For narrow resonances, which we define as $\Gamma_{Z^{\prime}}<0.1 M_{Z^{\prime}}$, interference effects can be neglected. This is an excellent approximation near the resonance peak. In the region slightly off peak, the interference terms can significantly alter the shape of the invariant mass distribution [6, 35].

The partial width for decay into a massless fermion pair $f \bar{f}$ is given by

$$
\Gamma_{Z^{\prime}}^{f}=\frac{M_{Z^{\prime}}}{24 \pi}\left(g_{f_{L}}^{2}+g_{f_{R}}^{2}\right)
$$

where the $g_{f_{L, R}}$ are the fermion couplings, which can be written in terms of the overall $Z^{\prime}$ coupling and the fermion charges $z_{f}$ :

$$
g_{f_{L, R}}^{2}=g_{Z^{\prime}}^{2} z_{f_{L, R}}^{2}=\frac{4 \pi \alpha}{\cos ^{2} \theta_{W}} z_{f_{L, R}}^{2}
$$

We take the masses of the quarks to be negligible compared to $M_{Z^{\prime}} / 2$. We assume for simplicity that the $Z^{\prime}$ decays only to SM fermions. However, in models with extra fermions, the $Z^{\prime}$ might also have non-SM decays. Thus, the total width is generally a free parameter. If the decay rate to new fermions is large, the $Z^{\prime}$ mass limits could be significantly relaxed due to the reduced SM branching fractions [36].

In scattering experiments, the $Z^{\prime}$ couplings influence the kinematics of the final state particles. Before discussing this further, we define some standard scattering variables. We choose a polar coordinate system about the beam axis, $z$, with azimuthal and polar 
angles $\phi$ and $\theta$, respectively. The transverse momentum (the momentum of a particle perpendicular to the beam line) is given by:

$$
p_{T}=\sqrt{p_{x}^{2}+p_{y}^{2}}=p \sin \theta
$$

and the transverse energy by:

$$
E_{T}=\sqrt{p_{T}^{2}+m^{2}}
$$

These quantities are equivalent for massless particles. Oftentimes, the polar angle $\theta$ is replaced by a related quantity known as pseudorapidty:

$$
\eta=-\ln \tan (\theta / 2)
$$

There are two quantities that are particularly relevant for $Z^{\prime}$ searches, namely the rapidity, $y$, and the forward-backward asymmetry, $A_{F B}$. The rapidity describes the boost along the beam axis of the CM frame relative to the lab frame, and is given by

$$
y=\frac{1}{2} \ln \left(\frac{E+p_{z}}{E-p_{z}}\right) .
$$

The forward-backward asymmetry is given by

$$
A_{F B}=\frac{F-B}{F+B}
$$

where $F[B]$ is the number of events where the final state fermion is scattered in the forward [backward], i.e. $\cos \theta>0[<0]$, direction relative to the initial quark direction. In Section 2.4, we will show how these two quantities can be used to diagnose the $Z^{\prime}$ couplings.

Dilepton resonances are not the only viable channel for $Z^{\prime}$ detection. Past work has considered detection using decays to top quarks [37, 38, 39, 40] and third generation fermions [41, 42, 43], weak boson pair production [44], and weak charge measurements in atomic parity violation experiments [45, 46]. The latter channels are particularly useful in the case of leptophobic or non-universal $Z^{\prime}$ models [47].

Other new physics, including Randall-Sundrum gravitons or sneutrinos, could also be detected via a dilepton resonance similar to a $Z^{\prime}$. There have been several discussions of how to differentiate such resonances from a $Z^{\prime}$ [40, 48]. 


\subsection{Simulation}

We have analyzed the characteristic features of $Z^{\prime}$ production at the LHC using an expanded version of the simulation code FEWZ [22, 23]. FEWZ allows for an extensive analysis of the SM Drell-Yan process, including NNLO effects and the influence of phase space cuts. With a few modifications, its features can be used to study $Z^{\prime}$ production as well. The details of the original simulation are provided in Refs. [22, 23].

The QCD factorization theorem allows us to write the $Z^{\prime}$ production cross section in terms of the partonic cross section and the proton structure functions as follows:

$$
d \sigma=\sum_{i j} \int d x_{1} d x_{2} f_{i}^{h_{1}}\left(x_{1}\right) f_{j}^{h_{2}}\left(x_{2}\right) d \sigma_{i j \rightarrow l_{1} l_{2}}\left(x_{1}, x_{2}\right)
$$

where $x_{1,2}$ is the fraction of the proton momentum carried by the quark. Since the addition of a $Z^{\prime}$ affects only the partonic cross section, and not the structure functions, we do not need to alter the Monte Carlo portion of FEWZ.

The parameters of the $Z^{\prime}$ model are specified in the input file for each run. For each model, the user sets the mass, total width, partial width to leptons, and couplings of the $Z^{\prime}$. The input file also includes the SM parameters and kinematic cuts. We also include a switch to turn on and off the $Z^{\prime}$ contribution, so that calculations of the SM background can still be done.

The $Z^{\prime}$ parameters are read into FEWZ and used to calculate weights (related to the partonic cross section of various subprocesses) for the integration routine. Preserving the structure of the SM calculation, we insert additional $Z^{\prime}$ contributions to the partonic cross section according to Eq. 2.1). Hereafter, the Monte Carlo integration proceeds without alteration. All calculations are done to NLO or NNLO in QCD using the MSTW2008 PDF sets [49]. Factorization and renormalization scales are set to $\mu_{F}=$ $\mu_{R}=M_{Z^{\prime}}[23]$. 
We adopt the following standard acceptance cuts in our analysis:

$$
\begin{aligned}
p_{T}^{\ell} & >20 \mathrm{GeV} \\
\left|\eta_{\ell}\right| & <2.5 \\
\left|y_{Z^{\prime}}\right| & >0.8 \text { (for } \mathrm{A}_{\mathrm{FB}} \text { only). }
\end{aligned}
$$

The first two of these cuts are the same as those applied to the muon channel by CMS and ATLAS [26, 25]. The third is used to define a forward-backward asymmetry. The intial quark direction cannot be measured directly at proton-proton colliders. However, the boost direction of the $Z^{\prime}$ is preferentially in the direction of the quark, not the antiquark, especially for large dilepton rapidity $\left|y_{l l}\right|$. Valence quarks are much more likely than any other partons to carry a large fraction of the proton momentum. Thus, if the dilepton rapidity is large, the boost is preferentially in the valence quark direction. However, for small dilepton rapidities, the initial momenta of the quark and antiquark have similar magnitudes, so we cannot use the parton distributions to distinguish between them. Therefore, placing a cut on the rapidity of the final dilepton system allows for a measurement of $A_{F B}$ [33].

We consider several common benchmark $Z^{\prime}$ models. The first, the sequential standard model (SSM), has couplings identical to those of the SM $Z$. It is a common benchmark in experimental searches. Another theoretically interesting case is the $Z_{B-L}^{\prime}$, noteworthy because $U(1)_{B-L}$ satisfies anomaly cancellation conditions without the presence of exotic fermions or non-universal couplings [6, 50, 51] and may be a remnant of string theory [52, 53, 54.

A theoretically well-motivated class of $Z^{\prime}$ models derives from breaking the $E_{6}$ gauge group via the chain [8]

$$
S U(5) \times U(1)_{\psi} \times U(1)_{\chi} \rightarrow S U(3)_{c} \times S U(2)_{L} \times U(1)_{Y} \times U(1)_{\psi} \times U(1)_{\chi}
$$

The new $U(1)$ factors are associated with two neutral gauge bosons, $Z_{\psi}$ and $Z_{\chi}$. After symmetry breaking, they mix to form the mass eigenstates $Z^{\prime}$ and $Z^{\prime \prime}$, with the mixing 
parameterized by an angle $\beta$ [55]:

$$
\begin{aligned}
& Z^{\prime}=Z_{\chi} \sin \beta+Z_{\psi} \cos \beta, \\
& Z^{\prime \prime}=Z_{\chi} \cos \beta-Z_{\psi} \sin \beta .
\end{aligned}
$$

Since the $Z^{\prime}$ and $Z^{\prime \prime}$ are assumed to be heavy compared to the SM $Z$-boson, any mixing with the $Z$ is negligible and ignored here. For now, we only consider the lower-mass $Z^{\prime}$ for the three specific cases $Z_{\psi}^{\prime}$ for $\beta=0, Z_{\chi}^{\prime}$ for $\beta=\pi / 2$, and $Z_{\eta}^{\prime}$ for $\tan \beta=\sqrt{3 / 5}$. We explore $Z^{\prime}$ and $Z^{\prime \prime}$ detection in this class of models in Section 2.5 .

Finally, we consider a left-right symmetric model, $Z_{L R}^{\prime}$ [56]. The $Z^{\prime}$ couples to the current

$$
J_{L R}^{\mu}=\alpha_{L R} J_{3 R}^{\mu}-\frac{1}{2 \alpha_{L R}} J_{B-L}^{\mu},
$$

where $\alpha_{L R}=\sqrt{\cos ^{2} \theta_{W} g_{R}^{2} / \sin ^{2} \theta_{W} g_{L}^{2}-1}$ and $\sin ^{2} \theta_{W}=0.22255$ [57]. We study an example $Z_{L R}^{\prime}$ with $g_{R}^{2}=g_{L}^{2}$ i.e., $\alpha_{L R} \approx 1.58$ [33].

Table 2.1 summarizes the $Z^{\prime}$ couplings to SM fermions for these models. The overall gauge coupling strength is a free parameter, but it is often chosen to be consistent with a grand unification scenario. We follow this approach and set $g_{Z^{\prime}}^{2}=\frac{4 \pi \alpha}{\cos ^{2} \theta_{W}}$ [32]. (This factor is not included in Table 2.1.)

In Table 2.2 we list for each model the total width, normalized by $M_{Z^{\prime}}$, as well as the branching fractions to leptons, quarks, and neutrinos, assuming no decays to non-SM particles. For our calculations, we use $\alpha^{-1}\left(M_{Z}\right)=128$.

In Fig. 2.1 we show the shape of the dilepton mass spectrum for a variety of $Z^{\prime}$ models with $M_{Z^{\prime}}=2.2 \mathrm{TeV}$ at $\sqrt{s}=8 \mathrm{TeV}$ (LHC8), and $M_{Z^{\prime}}=2.5 \mathrm{TeV}$ at $\sqrt{s}=$ $14 \mathrm{TeV}$ (LHC14). The SM background is very small in the resonance mass range, so for luminosities of $20 \mathrm{fb}^{-1}$ for $\sqrt{s}=8 \mathrm{TeV}$ or $100 \mathrm{fb}^{-1}$ for $\sqrt{s}=14 \mathrm{TeV}$, these resonance peaks should be clearly distinguishable. Distributions are calculated at next-to-nextto-leading order. We neglect detector effects such as energy resolution smearing. For ATLAS, the resolution width is about $1 \%$ for electrons and $5 \%$ for muons [25]. For CMS, the resolutions widths are $1-2 \%$ for electrons and $4-7 \%$ for muons [26]. As 


\begin{tabular}{|c|c|c|c|c|c|c|c|}
\hline & $z_{L}^{u}$ & $z_{L}^{d}$ & $z_{L}^{e}$ & $z_{R}^{u}$ & $z_{R}^{d}$ & $z_{R}^{e}$ & $z_{L}^{\nu}$ \\
\hline \hline & $\frac{\sqrt{10}}{12}$ & $\frac{\sqrt{10}}{12}$ & $\frac{\sqrt{10}}{12}$ & $\frac{-\sqrt{10}}{12}$ & $\frac{-\sqrt{10}}{12}$ & $\frac{-\sqrt{10}}{12}$ & $\frac{\sqrt{10}}{12}$ \\
$\chi$ & $\frac{-1}{2 \sqrt{6}}$ & $\frac{-1}{2 \sqrt{6}}$ & $\frac{3}{2 \sqrt{6}}$ & $\frac{1}{2 \sqrt{6}}$ & $\frac{-3}{2 \sqrt{6}}$ & $\frac{1}{2 \sqrt{6}}$ & $\frac{3}{2 \sqrt{6}}$ \\
$\eta$ & $\frac{1}{3}$ & $\frac{1}{3}$ & $\frac{-1}{6}$ & $\frac{-1}{3}$ & $\frac{1}{6}$ & $\frac{-1}{3}$ \\
$B-L$ & $\frac{\sqrt{5}}{6 \sqrt{2}}$ & $\frac{\sqrt{5}}{6 \sqrt{2}}$ & $-\frac{\sqrt{5}}{2 \sqrt{2}}$ & $\frac{\sqrt{5}}{6 \sqrt{2}}$ & $\frac{\sqrt{5}}{6 \sqrt{2}}$ & $-\frac{\sqrt{5}}{2 \sqrt{2}}$ & $-\frac{\sqrt{5}}{2 \sqrt{2}}$ \\
$\mathrm{LR}$ & $-\frac{1}{6 \alpha_{L R}}$ & $-\frac{1}{6 \alpha_{L R}}$ & $\frac{1}{2 \alpha_{L R}}$ & $-\frac{1}{6 \alpha_{L R}}+\frac{\alpha_{L R}}{2}$ & $-\frac{1}{6 \alpha_{L R}}-\frac{\alpha_{L R}}{2}$ & $\frac{1}{2 \alpha_{L R}}-\frac{\alpha_{L R}}{2}$ & $\frac{1}{2 \alpha_{L R}}$ \\
\hline
\end{tabular}

Table 2.1 Z' gauge charges. For the LR model, $\alpha_{L R} \approx 1.58$ 


\begin{tabular}{|c|c|c|c|c|c|}
\hline Model & $\Gamma / M_{Z^{\prime}}$ & $\mathrm{BF}\left(\mu^{+} \mu^{-}\right)$ & $\mathrm{BF}(t \bar{t})$ & $\mathrm{BF}$ (hadrons) & $\mathrm{BF}(\nu \bar{\nu})$ \\
\hline \hline$\psi$ & 0.005 & 0.04 & 0.12 & 0.80 & 0.07 \\
$\chi$ & 0.012 & 0.06 & 0.03 & 0.65 & 0.16 \\
$\eta$ & 0.006 & 0.04 & 0.16 & 0.87 & 0.02 \\
$B-L$ & 0.014 & 0.15 & 0.05 & 0.31 & 0.23 \\
$\mathrm{LR}$ & 0.022 & 0.03 & 0.10 & 0.90 & 0.02 \\
$\mathrm{SSM}$ & 0.026 & 0.03 & 0.10 & 0.73 & 0.18 \\
\hline
\end{tabular}

Table 2.2 $Z^{\prime}$ decay widths and branching fractions, assuming no non-SM fermion decays.

can be seen in Table 2.2, the resolutions are generally comparable to or larger than the decay widths for the $Z^{\prime}$ (assuming that non-SM decay rates are not large). Therefore a precise measurement of the $Z^{\prime}$ width will likely be difficult [35]. As we show later, there are observables that do not depend strongly on the decay width but still provide useful information about the properties of the $Z^{\prime}$.

By integrating over the peak region, we can determine the cross section as a function of $M_{Z^{\prime}}$ for each model. In Fig. 2.2, we show the mass dependence of the integrated peak $\left( \pm 3 \Gamma_{Z^{\prime}}\right)$ cross section for our six model examples at NLO. Note that the mass dependence is largely model-independent.

\subsection{Analysis}

We now present a few empirical formulas from our simulations, which allow the cross section and the differential distributions over a wide range of model parameters to be predicted. They are also useful in extracting coupling information from experimental data. 

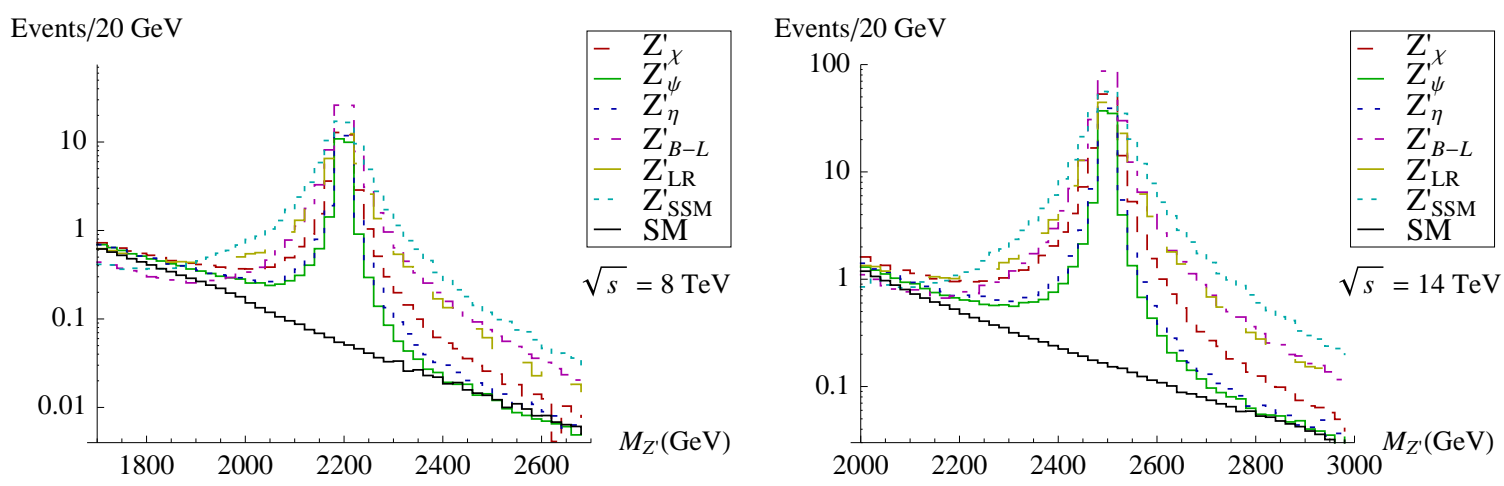

Figure 2.1 Simulated NNLO dimuon invariant mass spectrum for a variety of $Z^{\prime}$ models with $M_{Z^{\prime}}=2.2 \mathrm{TeV}$ and $\sqrt{s}=8 \mathrm{TeV}$ (left) and $M_{Z^{\prime}}=2.5 \mathrm{TeV}$ and $\sqrt{s}=14 \mathrm{TeV}$ (right), both with a luminosity of $\mathcal{L}=100 \mathrm{fb}^{-1}$.

The LHC production cross section of the $Z^{\prime}$ depends on the mass, width, and couplings. Under the assumption of generational universality, this dependence can be parameterized in the narrow width approximation by

$$
\sigma\left(p p \rightarrow Z^{\prime} \rightarrow l^{+} l^{-}\right)=\left[p\left(g_{u_{L}}^{2}+g_{u_{R}}^{2}\right)+(1-p)\left(g_{d_{L}}^{2}+g_{d_{R}}^{2}\right)\right] \mathrm{B}\left(Z^{\prime} \rightarrow l^{+} l^{-}\right) f\left(r_{Z^{\prime}}\right)
$$

The parameter $p$ is model-independent but varies with $r_{Z^{\prime}}$. It quantifies the fractional contribution to the cross section from up-type quark events. The dependence on the $Z^{\prime}$ mass is contained in $f\left(r_{Z^{\prime}}\right)$. We use the empirical representation [7],

$$
f\left(r_{Z^{\prime}}\right)=\sigma_{0} r_{Z^{\prime}}^{a}\left(\frac{1}{r_{Z^{\prime}}}-1\right)^{b}
$$

of the structure function dependence, where $r_{Z^{\prime}}=\frac{M_{Z^{\prime}}}{\sqrt{s}}$.

As in Refs. [32, 6], we define the quantities

$$
\begin{aligned}
c_{q} & =\frac{M_{Z}^{\prime}}{24 \pi \Gamma_{Z^{\prime}}}\left(g_{q_{L}}^{2}+g_{q_{R}}^{2}\right)\left(g_{e_{L}}^{2}+g_{e_{R}}^{2}\right)=\left(g_{q_{L}}^{2}+g_{q_{R}}^{2}\right) \mathrm{B}\left(Z^{\prime} \rightarrow l^{+} l^{-}\right), \\
e_{q} & =\frac{M_{Z}^{\prime}}{24 \pi \Gamma_{Z^{\prime}}}\left(g_{q_{L}}^{2}-g_{q_{R}}^{2}\right)\left(g_{e_{L}}^{2}-g_{e_{R}}^{2}\right) .
\end{aligned}
$$



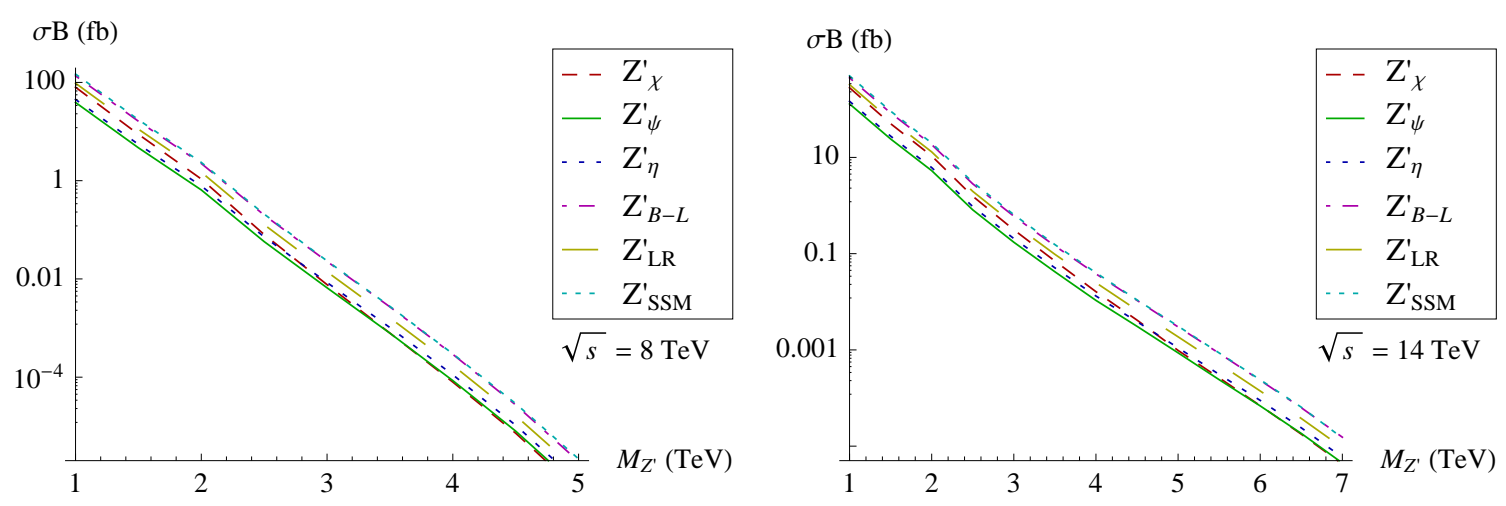

Figure 2.2 NLO cross section for the process $Z^{\prime} \rightarrow l^{+} l^{-}$versus $Z^{\prime}$ mass, integrated over the dilepton invariant mass peak region $( \pm 3 \Gamma)$ for $\sqrt{s}=8 \mathrm{TeV}$ (left) and $\sqrt{s}=14 \mathrm{TeV}$ (right).

In this notation, the cross section formula takes the simple form:

$$
\sigma\left(p p \rightarrow Z^{\prime} \rightarrow l^{+} l^{-}\right)=\left[p c_{u}+(1-p) c_{d}\right] f\left(r_{Z^{\prime}}\right)
$$

We use a NLO FEWZ simulation of the six model examples in fits of the parameters of Eqs. (2.13) and 2.14). First, we fix $r_{Z^{\prime}}$ and fit the simulated cross sections for the six models to the form $A c_{u}+B c_{d}$, then take $p=\frac{A}{A+B}$. We repeat this procedure for several values of $r_{Z^{\prime}}$ over the range, $0.1-0.6\left(M_{Z^{\prime}}=1.0-4.5 \mathrm{TeV}\right.$ for $\sqrt{s}=8 \mathrm{TeV}$; $M_{Z^{\prime}}=2.0-7.0 \mathrm{TeV}$ for $\left.\sqrt{s}=14 \mathrm{TeV}\right)$, to determine how $p$ changes with $r_{Z^{\prime}}$. We find that this dependence can be approximated by the function

$$
p\left(r_{Z^{\prime}}\right)=0.77-0.17 \tan ^{-1}\left(2.6-9.5 r_{Z^{\prime}}\right) .
$$

The result is shown in Fig. 2.3.

To determine $f\left(r_{Z^{\prime}}\right)$, we fit the normalization $A+B$ to the form given in Eq. (2.14). Our best fit values are

$$
f\left(r_{Z^{\prime}}\right)= \begin{cases}(3200 \mathrm{fb}) r_{Z^{\prime}}^{15.0}\left(\frac{1}{r_{Z^{\prime}}}-1\right)^{17.5} & \sqrt{s}=8 \mathrm{TeV} \\ (43.3 \mathrm{fb}) r_{Z^{\prime}}^{13.1}\left(\frac{1}{r_{Z^{\prime}}}-1\right) & \sqrt{s}=14 \mathrm{TeV}\end{cases}
$$

A comparison of our fit to the simulation is plotted in Fig. 2.4. This fit captures the dependence on $r_{Z^{\prime}}$ within $20 \%$ over the entire range $M_{Z^{\prime}}=1-7 \mathrm{TeV}$. 


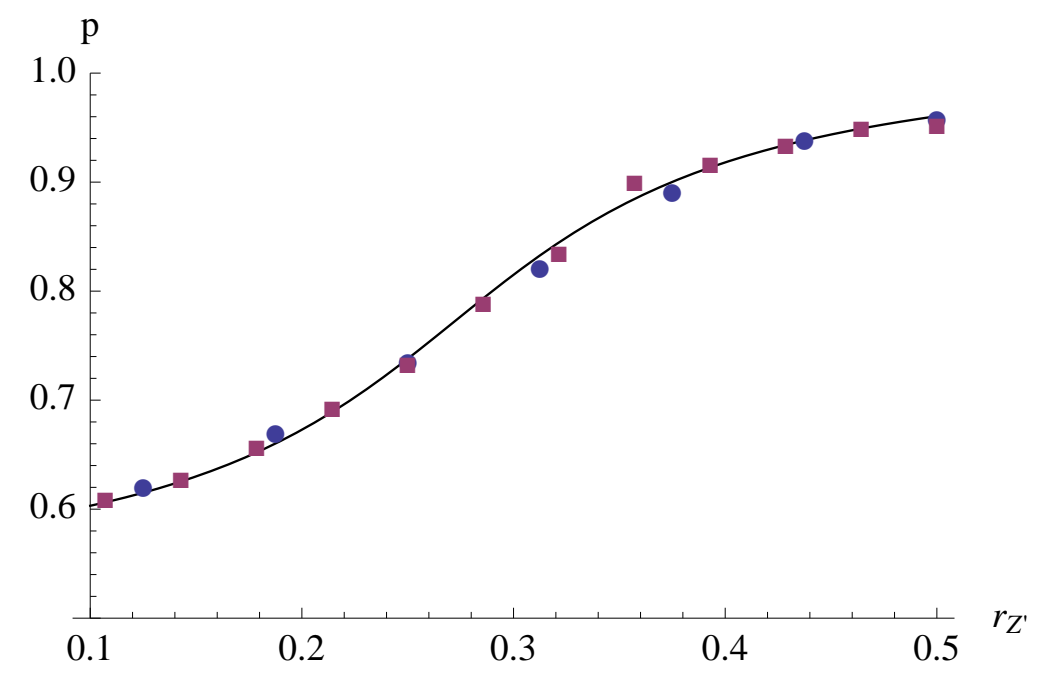

Figure 2.3 The parameter $p$ (as defined in Eq. 2.13) as a function of $r_{Z^{\prime}}=\frac{M_{Z^{\prime}}}{\sqrt{s}}$, along with an approximate fit to an arctangent function. Notice that simulated values (points) are shown for both $8 \mathrm{TeV}$ (squares) and $14 \mathrm{TeV}$ (circles) and agree well.

After a $Z^{\prime}$ is detected, the next step will be to measure its couplings. Measuring couplings with precision requires a large number of events, which may not be the situation for a very heavy or weakly coupled $Z^{\prime}$. However, with enough events, an accurate measurement of $c_{q}$ and $e_{q}$ is possible, as we demonstrate by considering a set of simulated measurements of a $2.5 \mathrm{TeV} Z^{\prime}$ at $\sqrt{s}=14 \mathrm{TeV}$.

The coupling combinations $c_{q}$ and $e_{q}$ of Eq. 2.15 can be determined by considering the differential cross section, integrated over the resonance peak:

$$
\begin{aligned}
\frac{d \sigma_{i n t}}{d y d \cos \theta}= & \int_{M_{Z^{\prime}}-3 \Gamma}^{M_{Z^{\prime}}+3 \Gamma} \frac{d \sigma}{d y d \cos \theta d Q} d Q \\
= & 3 / 8\left(1+\cos ^{2} \theta\right)\left[p c_{u} h_{1}^{u}(y)+(1-p) c_{d} h_{1}^{d}(y)\right] f\left(r_{Z^{\prime}}\right) \\
& +\cos \theta\left[p e_{u} h_{2}^{u}(y)+(1-p) e_{d} h_{2}^{d}(y)\right] g\left(r_{Z^{\prime}}\right) .
\end{aligned}
$$

The functions $h_{1,2}^{q}$ are normalized so that integrating over $\cos \theta$ and $y$ yields Eq. 2.17). $g\left(r_{Z^{\prime}}\right)$ represents the mass dependence of the $\cos \theta$ term, similar to $f\left(r_{Z^{\prime}}\right)$. For $M_{Z^{\prime}}=$ $2.5 \mathrm{TeV}$, its value is $g\left(r_{Z^{\prime}}\right)=506 \mathrm{fb}$. For the same mass, we find $f\left(r_{Z^{\prime}}\right)=1050 \mathrm{fb}$. 

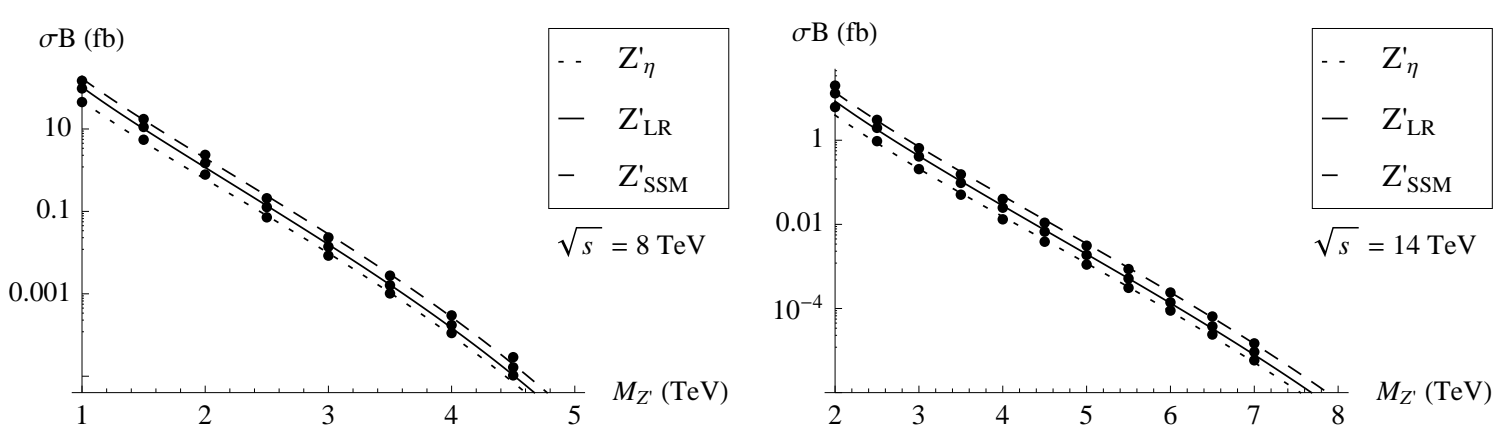

Figure 2.4 FEWZ simulation (points) and fitted curve (lines) of the integrated $Z^{\prime}$ cross section for $\sqrt{s}=8 \mathrm{TeV}$ (left) and $\sqrt{s}=14 \mathrm{TeV}$ (right). We show only the $E_{6} \eta$, left-right, and sequential models for simplicity.

We have already determined $p, f\left(r_{Z^{\prime}}\right)$, and $g\left(r_{Z^{\prime}}\right)$, so all that remains is to find $h_{1,2}^{q}$. In order to separate the up and down contributions to the differential cross section, we define two distinct scenarios in which the $Z^{\prime}$ couples exclusively to one type of quark (and to leptons). Then, for each scenario we simulate two differential distributions. The first is the dilepton rapidity distribution, which allows us to determine $h_{1}^{q}$. To determine $h_{2}^{q}$, instead of using the total cross section, we consider the quantity $\frac{d(F-B)}{d y}$, where $F$ is the number of lepton pairs scattered in the forward $\left(\cos \theta_{Z^{\prime}}>0\right)$ direction in the Collins-Soper frame [58] and $B$ is the number scattered in the backward direction. The resulting distributions are easily distinguishable, as can be seen in Fig. 2.5. In addition to the simulated NLO data, Fig. 2.5 shows approximate curves $h_{1,2}^{q}(y)$. The specific curves used to approximate the normalized distributions at $14 \mathrm{TeV}$ are

$$
\begin{aligned}
h_{1}^{u}(y) & =\frac{0.59}{e^{4.6(y-0.84)}+1}, \\
h_{1}^{d}(y) & =\frac{0.78}{e^{4.6(y-0.63)}+1}, \\
h_{2}^{u}(y) & =\frac{2.5\left(1-e^{-0.60 y}\right)}{e^{5.7(y-0.84)}+1}, \\
h_{2}^{d}(y) & =\frac{11\left(1-e^{-0.20 y}\right)}{e^{5.8(y-0.63)}+1} .
\end{aligned}
$$

By fitting observed data to Eq. (2.20), one can determine the four coefficients $c_{q}$ and $e_{q}$. To demonstrate the feasibility of this method and estimate the statistical error, we 

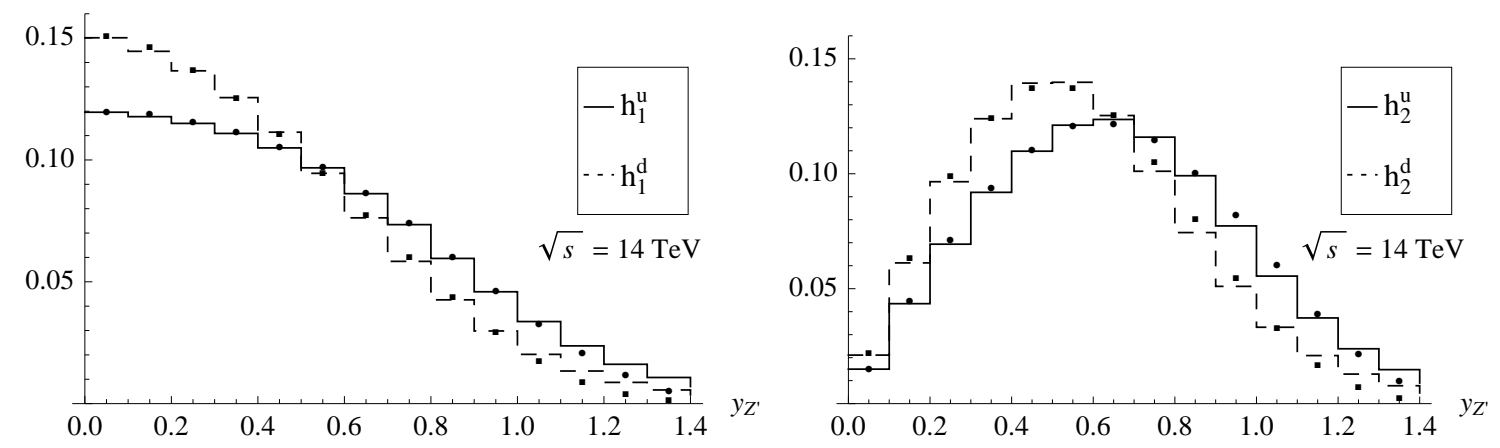

Figure 2.5 Normalized distributions for $\frac{d \sigma}{d y}$ (left) and $\frac{d(F-B)}{d y}$ (right) for a $Z^{\prime}$ that couples to only up-type (solid) or down-type (dashed) quarks. We have set $M_{Z^{\prime}}=2.5 \mathrm{TeV}$ at LHC14. Points are simulated data and lines are fitted curves.

use our simulation as a pseudo-experiment. For each reference model in Section 2.3 , we generate binned distributions for $\frac{d N}{d y}$ and $\frac{d(F-B)}{d y}$. To extract $c_{q}$ and $e_{q}$, we fit these distributions to linear combinations of $h_{1,2}^{u}$ and $h_{1,2}^{d}$ by minimizing $\chi^{2}$. To determine the boundaries of the confidence regions, we vary $c_{q}$ and $e_{q}$ and calculate the $\chi^{2}$ value at each point for an "average" experiment using the method described in Appendix A of [59].

Figures 2.6 and 2.7 show the $95 \%$ confidence level (C.L.) regions for our example case of a $2.5 \mathrm{TeV} Z^{\prime}$ at $14 \mathrm{TeV}$. We see that $100 \mathrm{fb}^{-1}$ allows for some model differentiation, while for $1 \mathrm{ab}^{-1}$ of luminosity the confidence regions are narrow. In the right panel of Fig. 2.6, the red dashed contour shows the values of $c_{u}$ and $c_{d}$ for the $E_{6}$ models as a function of the mixing angle $\beta$, from which we can see that some model differentiation should be possible. The tilt of the ellipses arises from the requirement that the up and down quark contributions are summed to give the total cross section, restricting $c_{u}$ and $c_{d}$ to lie on a line.

Within the $E_{6}$ model class, our fit can also be used to place limits on $\beta$ using the least squares method. Using Eqs. (2.11) and (2.15), we can write Eq. (2.20) in terms of 

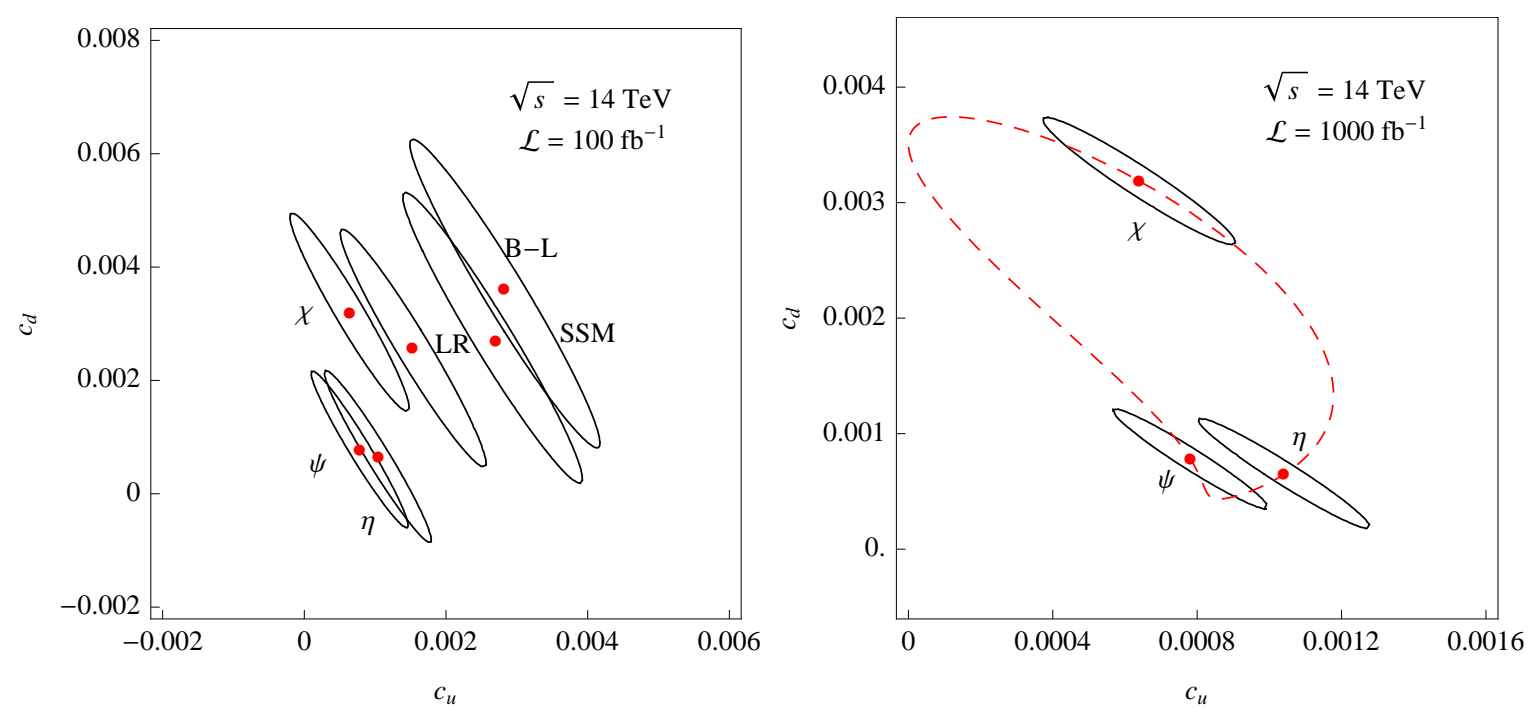

Figure 2.6 95\% C.L. regions for the couplings $c_{u}$ and $c_{d}$ for an average experiment (statistical errors only) for $\mathcal{L}=100 \mathrm{fb}^{-1}$ (left) and $\mathcal{L}=1 \mathrm{ab}^{-1}$ (right). The values of $c_{u}$ and $c_{d}$ for the $E_{6}$ family lie on the dashed red contour. Points are the theoretical values.

$\beta$ instead of $c_{q}$ and $e_{q}$. At the 1-sigma level, we find

$$
\begin{aligned}
& 0.44 \pi<\beta_{\chi}<0.54 \pi \\
& 0.97 \pi<\beta_{\psi}<1.03 \pi \text { or } 1.14 \pi<\beta_{\psi}<1.17 \pi \\
& 0.20 \pi<\beta_{\eta}<0.22 \pi
\end{aligned}
$$

If the number of events is low, an analysis can still be done by integrating over the distributions $h_{1,2}^{q}$, leading to a system of four equations in four unknowns that can be inverted [32]. Note that we have included only statistical errors. For information on the effect of PDF errors, see Ref. [32].

From our fit, we can also determine the forward-backward asymmetry,

$$
A_{F B}=\frac{F-B}{F+B}
$$



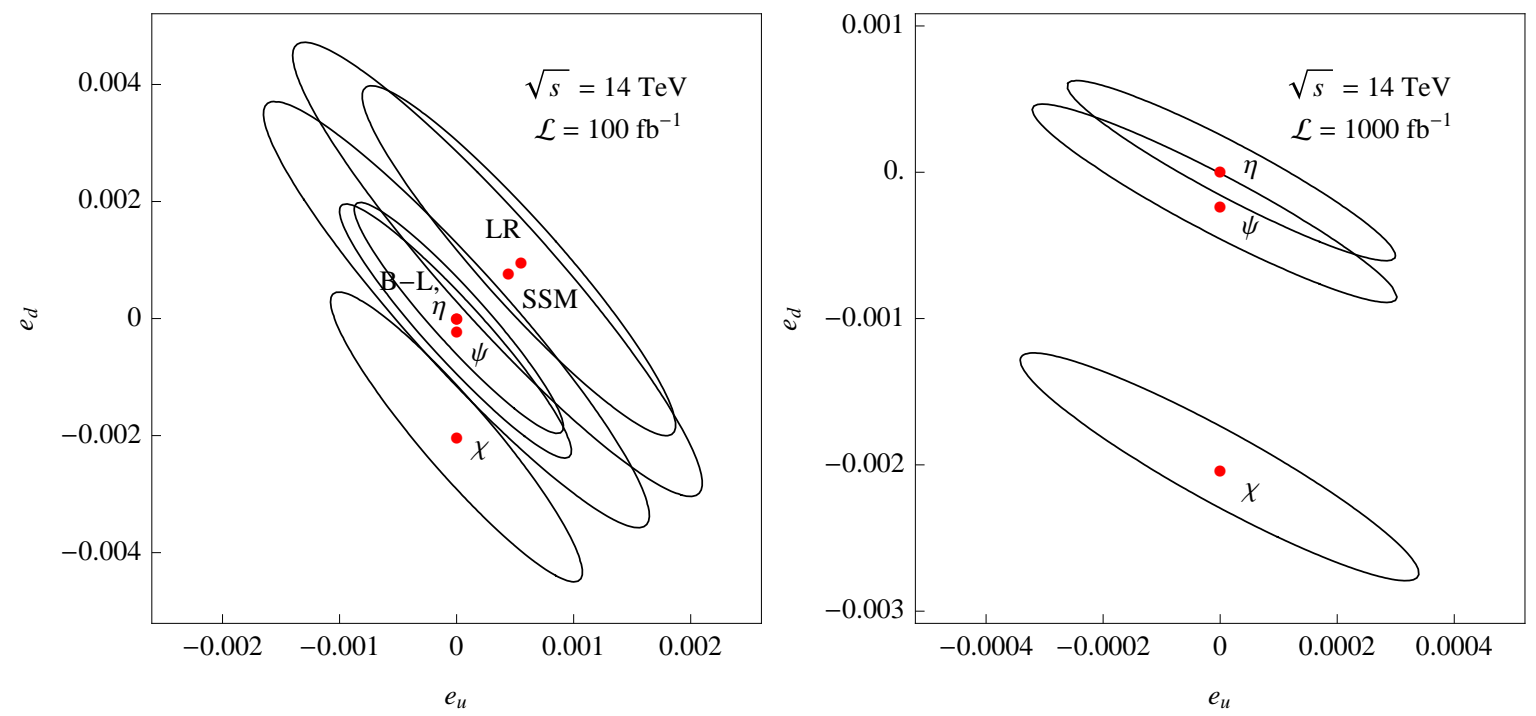

Figure $2.795 \%$ confidence level regions for the couplings $e_{u}$ and $e_{d}$ for an average experiment for $\mathcal{L}=100 \mathrm{fb}^{-1}$ (left) and $\mathcal{L}=1 \mathrm{ab}^{-1}$ (right). Points are the theoretical values.

On the $Z^{\prime}$ peak, $A_{F B}$ depends solely on the $Z^{\prime}$ couplings to fermions. Using Eq. 2.20), integrated over appropriate ranges of $\cos \theta$, we can write

$$
A_{F B}=\frac{\left(p a_{2}^{u} e_{u}+(1-p) a_{2}^{d} e_{d}\right)}{\left(p a_{1}^{u} c_{u}+(1-p) a_{1}^{d} c_{d}\right)} \frac{g\left(M_{Z^{\prime}}\right)}{f\left(M_{Z^{\prime}}\right)},
$$

where $a_{1,2}^{q}=2 \int_{y_{\min }}^{y_{\max }} h_{1,2}^{q}(y) d y$. We choose $y_{\max }=2.5$ and $y_{\min }=0.8$, the rapidity cuts discussed in Section 2.3.

In the left panel of Fig. 2.8, we show the simulated values of $A_{F B}$ along with statistical uncertainties for three $E_{6}$ models with $100 \mathrm{fb}^{-1}$ of data. The red curve shows the predicted values for $A_{F B}$ versus the mixing angle $\beta$ defined in Eq. (2.11). The couplings of the $L R, B-L$, and $\chi$ models can also be parameterized (up to a normalization factor) by an angle $\alpha$ with [28]

$$
Z^{\prime}=\cos \alpha Z_{\chi}+\sin \alpha Z_{Y}
$$

This is equivalent to

$$
Z_{L R}^{\prime}=\cos \theta_{L R}\left(-Z_{B-L}\right)+\sin \theta_{L R} Z_{R}
$$

where $\arctan \left(\alpha_{L R}\right)=\theta_{L R}=\alpha+\arctan \sqrt{2 / 3}$. As for the three $E_{6}$ models, we show $A_{F B}$ versus $\alpha$ in the right panel of Fig 2.8 . 

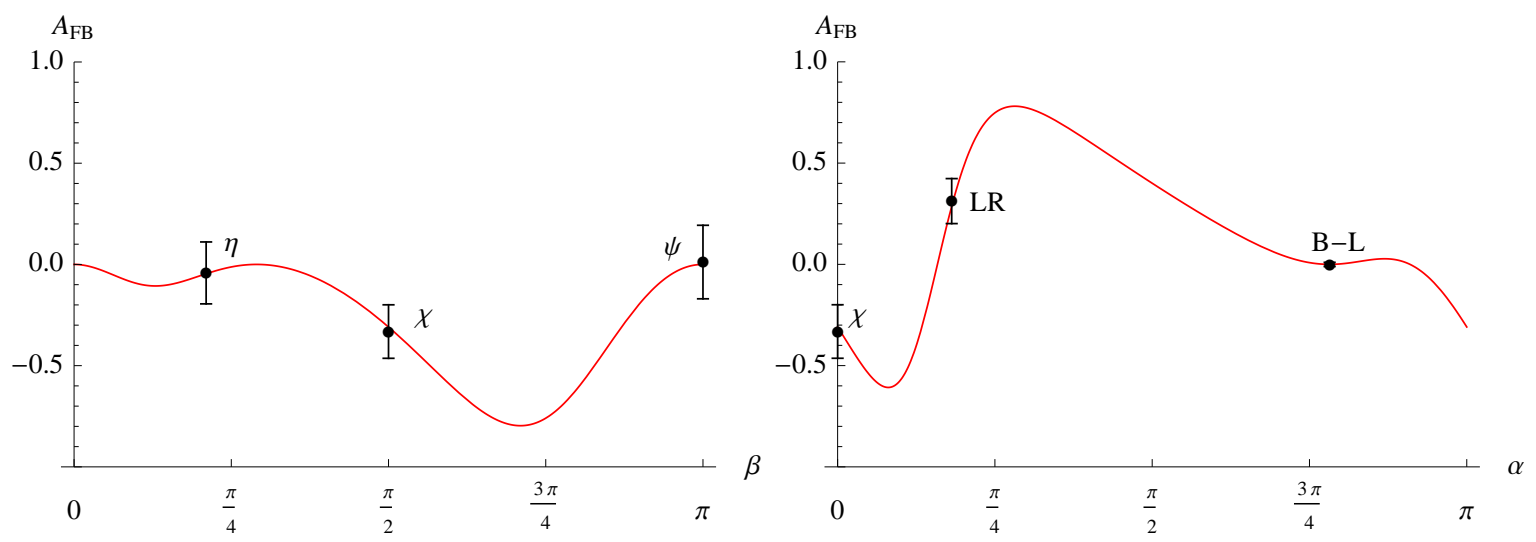

Figure 2.8 Predicted on-peak forward-backward asymmetry versus mixing angle $\beta$ for the $E_{6}$ models (left) and $\alpha$ for the LR models (right), along with FEWZ simulated values and statistical errors for $M_{Z^{\prime}}=2.5 \mathrm{TeV}, \sqrt{s}=14 \mathrm{TeV}$, and $\mathcal{L}=100 \mathrm{fb}^{-1}$.

Off peak, the $Z^{\prime}$ interference with the SM background contributes to the asymmetry, so $A_{F B}$ varies significantly with dilepton mass. Figure 2.9 shows the effect of this interference on $A_{F B}$. Both the shape of the curve and the peak value are highly modeldependent.

\section{$2.5 \quad E_{6}$ Models}

We now consider $E_{6}$ grand unification scenarios in more detail. An $E_{6}$ gauge group can be broken down into either a rank- 5 or rank- 6 subgroup. In the rank- 5 case, this leads to one additional $Z^{\prime}$ boson, the $Z_{\eta}^{\prime}$ discussed above. In the rank-6 case, there are two additional $Z^{\prime}$ s, corresponding to the additional $U(1)_{\psi}$ and $U(1)_{\chi}$ groups in Eq. (2.11). The mass eigenstates are $Z^{\prime}$ and $Z^{\prime \prime}$ of Eq. 2.11). We justifiably ignore small mixings of the $Z^{\prime}$ bosons with the SM $Z$. Often, the $Z^{\prime \prime}$ is assumed to be very heavy, leading to an effective rank-5 group, as was the case in the models we considered in Section 2.3 . In Fig. 2.10, we show the branching fractions and total width of each additional boson as a function of the mixing angle $\beta$. 


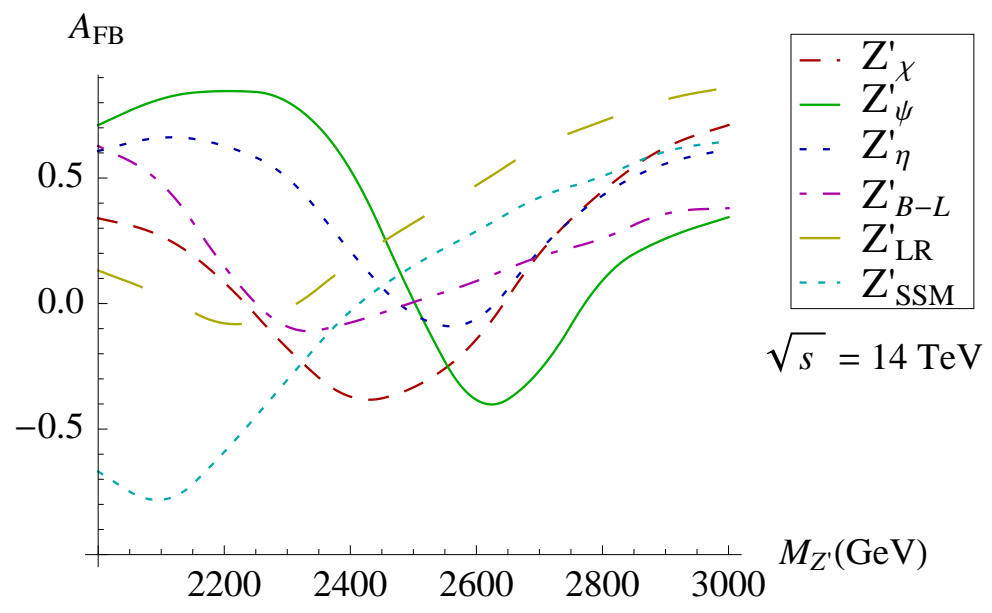

Figure 2.9 Simulated forward-backward asymmetry for a number of $Z^{\prime}$ models with $M_{Z^{\prime}}=2.5 \mathrm{TeV}$ and $\sqrt{s}=14 \mathrm{TeV}$.

In the rank-6 case, the masses of the $Z^{\prime}$ and $Z^{\prime \prime}$ are related by [55],

$$
\left(\frac{M_{Z^{\prime}}}{M_{Z^{\prime \prime}}}\right)^{2}=\left(\frac{\cos \beta+\sqrt{15} \sin \beta}{\sqrt{15} \cos \beta-\sin \beta}\right)\left(\frac{\cos \beta}{\sin \beta}\right) .
$$

This relation assumes that the $U(1)^{\prime}$ symmetry breaking scale is much higher than the electroweak scale. Since the experimental lower bound on the $Z^{\prime}$ mass is currently about $2 \mathrm{TeV}$ for $E_{6}$ models, and there are tight limits on mixing with the $\mathrm{SM} Z$, this is a justified assumption.

Requiring that the $Z^{\prime \prime}$ be heavier than the $Z^{\prime}$, and that both masses are positive leads to the condition

$$
-\sqrt{15} / 4 \leq \cos \beta \leq 0
$$

Notice that the $Z_{\eta}^{\prime}$, with $\cos \beta=\sqrt{5 / 8}$ is excluded from the range in Eq. 2.28. Additionally, both the $Z_{\psi}^{\prime}$ and $Z_{\chi}^{\prime}$, with $\cos \beta=1$ and $\cos \beta=0$, respectively, have $M_{Z^{\prime}} \ll M_{Z^{\prime \prime}}$. Therefore, we would not expect the LHC to detect a heavier mass eigenstate for the three $E_{6}$ models considered in Section 2.3 . 

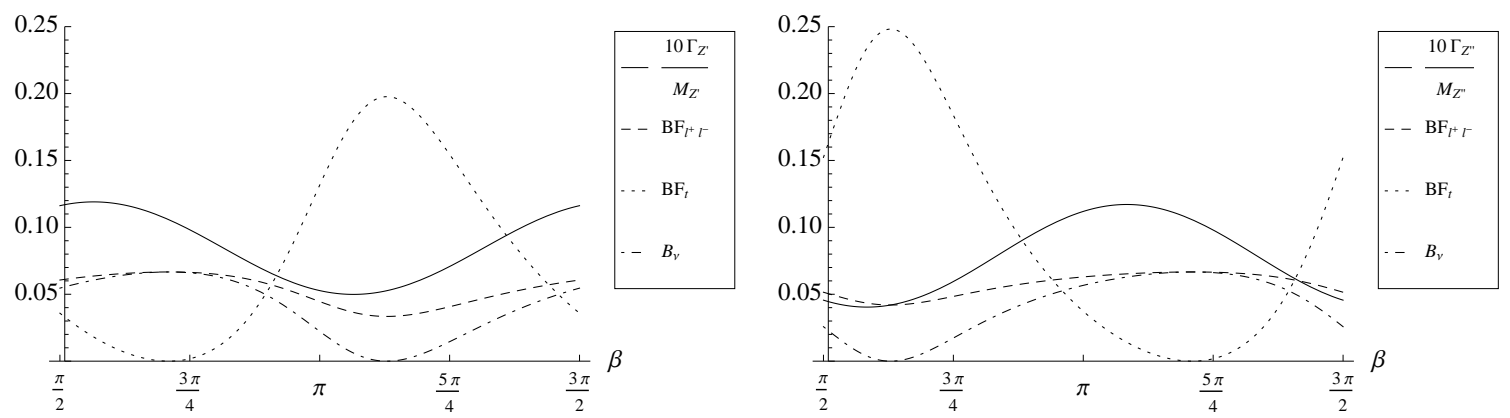

Figure 2.10 Total width, normalized by the mass, and branching fractions to lepton, top, and neutrino pairs for the lower mass eigenstate $Z^{\prime}$ (left) and the higher mass eigenstate $Z^{\prime \prime}$ (right). For both, we include only decays into SM fermions.

Using the empirical equations determined in Section 2.4, we can now calculate the cross section for the $Z^{\prime}$ and $Z^{\prime \prime}$. Both cross sections are a function of just two free parameters: $M_{Z^{\prime}}$ and $\beta$. In Fig. 2.11, we show the integrated peak cross section for $Z^{\prime}$ production and decay at LHC14 versus mixing angle. In Fig. 2.12, we plot the same quantity for the $Z^{\prime \prime}$ over the allowed range of mixing angles. Here we see that if a $Z^{\prime}$ were to be discovered in the mass range of $1-2.5 \mathrm{TeV}$ or so, the higher mass $Z^{\prime \prime}$ could be accessible at the LHC as well, within a certain range of mixing angles.

\subsection{Dark Matter Interactions}

There are a number of scenarios in which a $Z^{\prime}$ boson can serve as a dark matter mediator [60, 61, 62, 63, 64]. These models often include mixing between the gauge bosons, leading to small effective couplings between dark matter and SM fermions [65, 66, 10, 67, 68, 69, 70. We study this possibility, paying particular attention to the possibility of isospin-violating dark matter scattering, which occurs naturally in the case of a $Z^{\prime}$ mediator.

We now consider a model with a new $U(1)^{\prime}$ and a new Dirac fermion that is charged only under $U(1)^{\prime}$ - this fermion will serve as our dark matter candidate. Interactions between the dark matter and SM particles are achieved through the kinetic and mass 


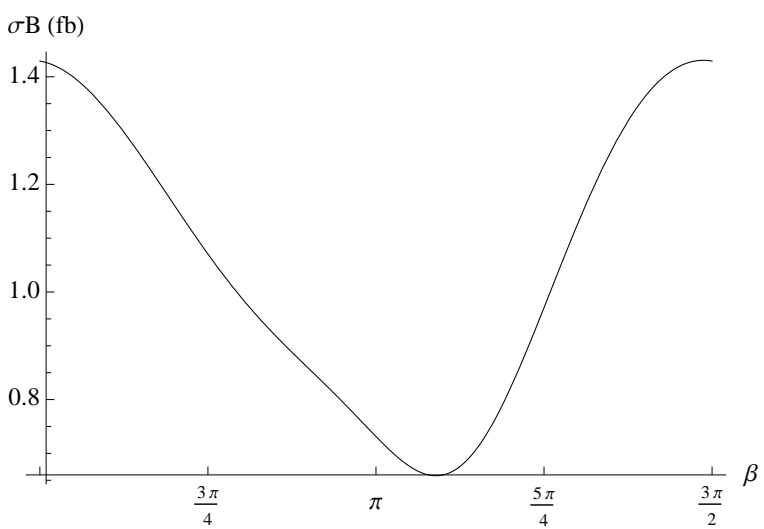

Figure 2.11 Integrated peak cross section for the process $p p \rightarrow Z^{\prime} \rightarrow l^{+} l^{-}$as a function of mixing angle $\beta$, with $M_{Z^{\prime}}=2.5 \mathrm{TeV}$ at LHC14.

mixing of the new $Z^{\prime}$ boson with the $\operatorname{SM~} Z$. The Lagrangian in this case is 66 ]

$$
\begin{aligned}
\mathcal{L}= & \mathcal{L}_{S M}-\frac{1}{4} \hat{Z}^{\prime}{ }_{\mu \nu} \hat{Z}^{\prime \mu \nu}+\frac{1}{2} M_{Z^{\prime}}^{2} \hat{Z}^{\prime}{ }_{\mu} \hat{Z}^{\prime \mu} \\
& -\hat{g}^{\prime} \sum_{i} \bar{\psi}_{i} \gamma^{\mu}\left(f_{V}^{i}-f_{A}^{i} \gamma^{5}\right) \bar{\psi}_{i} \hat{Z}_{\mu}^{\prime}-\frac{\sin \epsilon}{2} \hat{Z}^{\prime}{ }_{\mu \nu} \hat{B}^{\mu \nu}+\delta M^{2} \hat{Z}^{\prime}{ }_{\mu} \hat{Z}^{\mu} .
\end{aligned}
$$

Here $\sin \epsilon$ and $\delta M^{2}$ parameterize the kinetic and mass mixing between the $Z^{\prime}$ and the $Z$. As usual, $\hat{B}_{\mu \nu}, \hat{W}_{\mu \nu}$ and $\hat{Z}_{\mu \nu}^{\prime}$ are the field strength tensors for $U(1)_{Y}, S U(2)_{L}$ and $U(1)^{\prime}$. $\psi_{i}$ are the fermion fields (including the dark matter), and $f_{V}^{i}$ and $f_{A}^{i}$ are the vector and axial charges of the fermions under $U(1)^{\prime}$. For simplicity, we consider the case where all SM fermions have $f_{V}^{i}=f_{A}^{i}=0$. This choice leads to rather weak couplings between SM fermions and the new $Z^{\prime}$, which avoids current LHC bounds on $Z^{\prime}$ production. $f_{V}^{\chi}$ must be nonzero to allow for spin-independent scattering of dark matter on nuclei.

We define two additional parameters for convenience:

$$
\begin{aligned}
\delta & =\frac{\delta M^{2}}{M_{\hat{Z}}^{2}}, \\
\tan 2 \xi & =\frac{-2 \cos \epsilon\left(\delta+\hat{s}_{W} \sin \epsilon\right)}{M_{\hat{Z}^{\prime}}^{2} / M_{\hat{Z}}^{2}-\cos ^{2} \epsilon+\hat{s}_{W}^{2} \sin ^{2} \epsilon+2 \delta \sin \epsilon},
\end{aligned}
$$

where $\hat{s}_{W}$ is the sine of the weak mixing angle. The $Z^{\prime}$ couplings to DM and the shifts in the $Z$ couplings are proportional to $\xi$. Since $\xi$ is approximately proportional to $M_{Z^{\prime}}^{-2}$ for $\delta, \epsilon \ll \frac{M_{Z^{\prime}}}{M_{Z}}$, these couplings must be small for heavy $Z^{\prime}$ s. 


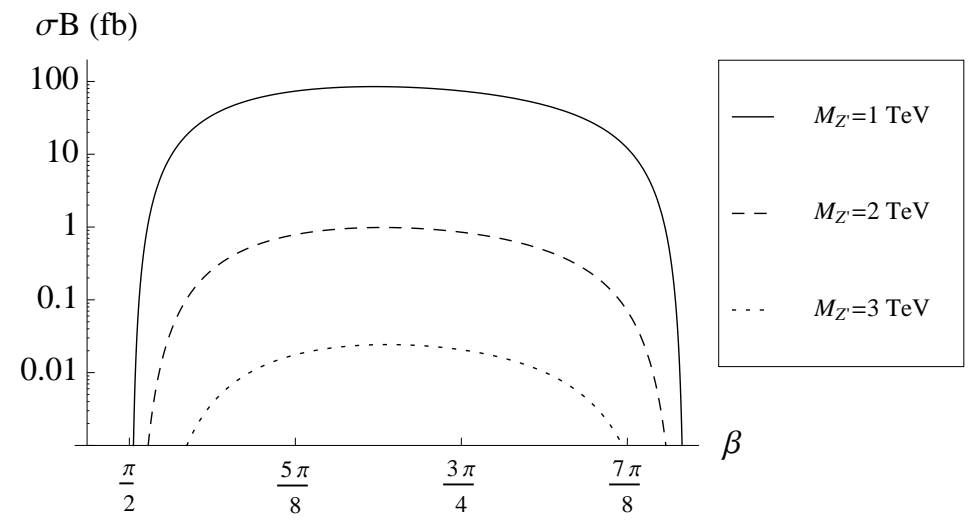

Figure 2.12 Integrated peak cross section for the process $p p \rightarrow Z^{\prime \prime} \rightarrow l^{+} l^{-}$at LHC14 versus mixing angle $\beta$. The mass of the heavier eigenstate is determined by the $Z^{\prime}$ mass and the mixing angle.

The physical states $A_{\mu}, Z_{\mu}$, and $Z_{\mu}^{\prime}$ are obtained through two sequential transformations. First, we diagonalize the field strength tensors; then, after $S U(2) \times U(1)$ breaking, we diagonalize the resulting mass matrices. After these transformations, the physical states are related to the original (hatted) states by

$$
\begin{aligned}
& A_{\mu}=\hat{A}_{\mu}+\hat{c}_{W} \sin \epsilon \hat{Z}_{\mu}^{\prime}, \\
& Z_{\mu}=\cos \xi\left(\hat{Z}_{\mu}-\hat{s}_{W} \sin \epsilon \hat{Z}_{\mu}^{\prime}\right)+\sin \xi \cos \epsilon \hat{Z}_{\mu}^{\prime}, \\
& Z_{\mu}^{\prime}=\cos \epsilon \cos \xi \hat{Z}_{\mu}^{\prime}-\sin \xi\left(\hat{Z}_{\mu}-\hat{s}_{W} \sin \epsilon \hat{Z}_{\mu}^{\prime}\right) .
\end{aligned}
$$


We can write the couplings of the physical states to fermions in terms of the oblique parameters, $S, T$, and $U$, and the "physical" weak mixing angle [66]:

$$
\begin{aligned}
g_{f Z}^{V} & =\frac{e}{2 s_{W} c_{W}}\left(1+\frac{\alpha T}{2}\right)\left(T_{3}^{i}-2 Q^{i} s_{*}^{2}\right), \\
g_{f Z}^{A} & =\frac{e}{2 s_{W} c_{W}}\left(1+\frac{\alpha T}{2}\right) T_{3}^{i}, \\
g_{f Z^{\prime}}^{V} & =\frac{e}{2 s_{W} c_{W}}\left(1+\frac{\alpha T}{2}\right)\left(\tilde{s}\left(T_{3}^{i}-2 Q^{i}\right) \tan \epsilon-\left(T_{3}^{i}-2 Q^{i} s_{*}^{2}\right) \xi\right), \\
g_{f Z^{\prime}}^{A} & =\frac{e}{2 s_{W} c_{W}}\left(1+\frac{\alpha T}{2}\right)\left(\tilde{s} T_{3}^{i} \tan \epsilon-T_{3}^{i} \xi\right), \\
g_{\chi Z}^{V} & =\xi f_{V}^{\chi}, \\
g_{\chi Z^{\prime}}^{V} & =f_{V}^{\chi},
\end{aligned}
$$

where

$$
s_{*}^{2}=s_{W}^{2}+\frac{1}{c_{W}^{2}-s_{W}^{2}}\left(\frac{1}{4} \alpha S-c_{W}^{2} s_{W}^{2} \alpha T\right),
$$

and

$$
\tilde{s}=s_{W}+\frac{s_{W}^{3}}{c_{W}^{2}-s_{W}^{2}}\left(\frac{1}{4 c_{W}^{2}} \alpha S-\frac{1}{2} \alpha T\right) .
$$

The contributions to $S$ and $T$ due to the $Z^{\prime}$ are, to second order in $\xi$ [66],

$$
\begin{gathered}
\alpha S=4 \xi c_{W}^{2} s_{W} \tan \epsilon \\
\alpha T=\xi^{2}\left(\frac{M_{Z^{\prime}}^{2}}{M_{Z}^{2}}-1\right)+2 \xi s_{W} \tan \epsilon .
\end{gathered}
$$

$S$ and $T$ are constrained by fits to the global electroweak data, as shown in Fig. 2.13 [57]. The best fit values for an assumed Higgs boson mass of $M_{H}=117 \mathrm{GeV}$ are

$$
\begin{aligned}
& S=0.03 \pm 0.09, \\
& T=0.07 \pm 0.08,
\end{aligned}
$$

with a strong correlation of $87 \%$. The $S$ and $T$ values would change very little for a Higgs mass of $125 \mathrm{GeV}$, as may be suggested by recent LHC observations [71, 72]. The constraints on $S$ and $T$ can be translated into constraints on the $Z^{\prime}$ mixing angles (and 

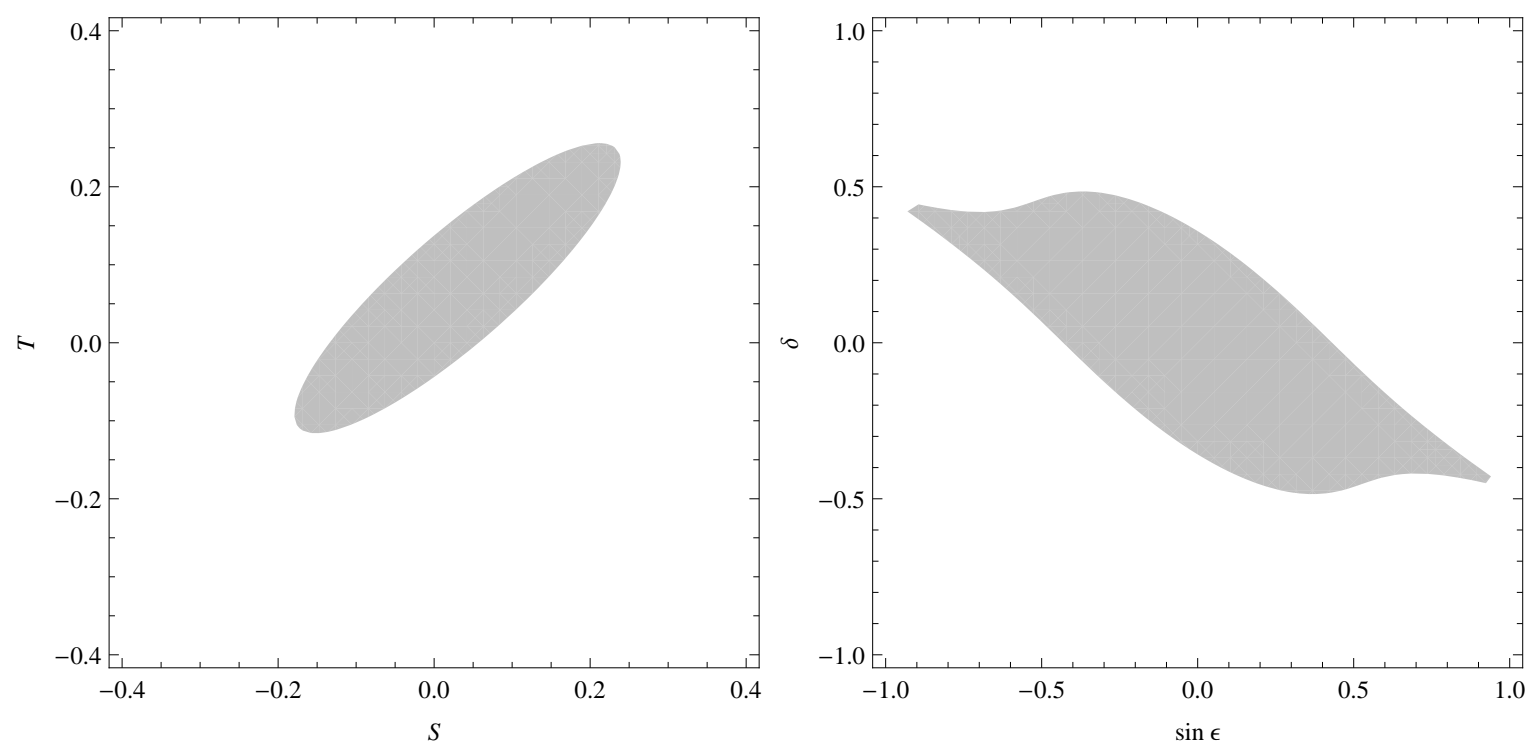

Figure 2.13 Left: The 90\% C.L. allowed region for $S$ and $T$ based on the global data for $M_{H}=117 \mathrm{GeV}$ [57]. Right: This region translated into a 90\% CL region for $\epsilon$ and $\delta$ for $M_{Z^{\prime}}=1000 \mathrm{GeV}$.

consequently the couplings and cross sections) with a simple Monte Carlo. The result is shown in Fig. 2.13. Note that the kinetic mixing angle $\epsilon$ can be quite large, assuming that $\delta$ is small enough.

By sampling values in the region of allowed $\epsilon$ and $\delta$, we can determine the range of possible values for the dark matter scattering cross section and the LHC $Z^{\prime}$ production cross section. These can then be compared to the results of experimental searches to further restrict the mixing angles, $Z^{\prime}$ mass, and $f_{n} / f_{p}$ (defined below).

For direct detection experiments, we are interested in interactions between dark matter and atomic nuclei [10]. To determine this cross section, we start with an effective dark matter-quark coupling given by

$$
b_{f}^{V, A}=\frac{g_{\chi Z^{\prime}}^{V, A} g_{f Z^{\prime}}^{V, A}}{M_{Z^{\prime}}^{2}}+\frac{g_{\chi Z}^{V, A} g_{f Z}^{V, A}}{M_{Z}^{2}}
$$

which leads to DM-nucleon couplings of

$$
\begin{gathered}
f_{n}=2 b_{d}^{V}+b_{u}^{V}, \\
f_{p}=b_{d}^{V}+2 b_{u}^{V} .
\end{gathered}
$$


Using these expressions, we can determine $f_{n} / f_{p}$ as a function of $\epsilon, \delta$, and $M_{Z^{\prime}}$. Contour plots of $f_{n} / f_{p}$ and $f_{p} / f_{n}$ as functions of $\epsilon$ and $\delta$ are shown in Fig. 2.14. Comparing with Fig. 2.13, we see that $S$ and $T$ place no limit on the value of $f_{n} / f_{p}$, though the mixing angles are more tightly constrained for some values of $f_{n} / f_{p}$ than others. Therefore, the limit on the dark matter scattering cross section will vary significantly as a function of $f_{n} / f_{p}$.
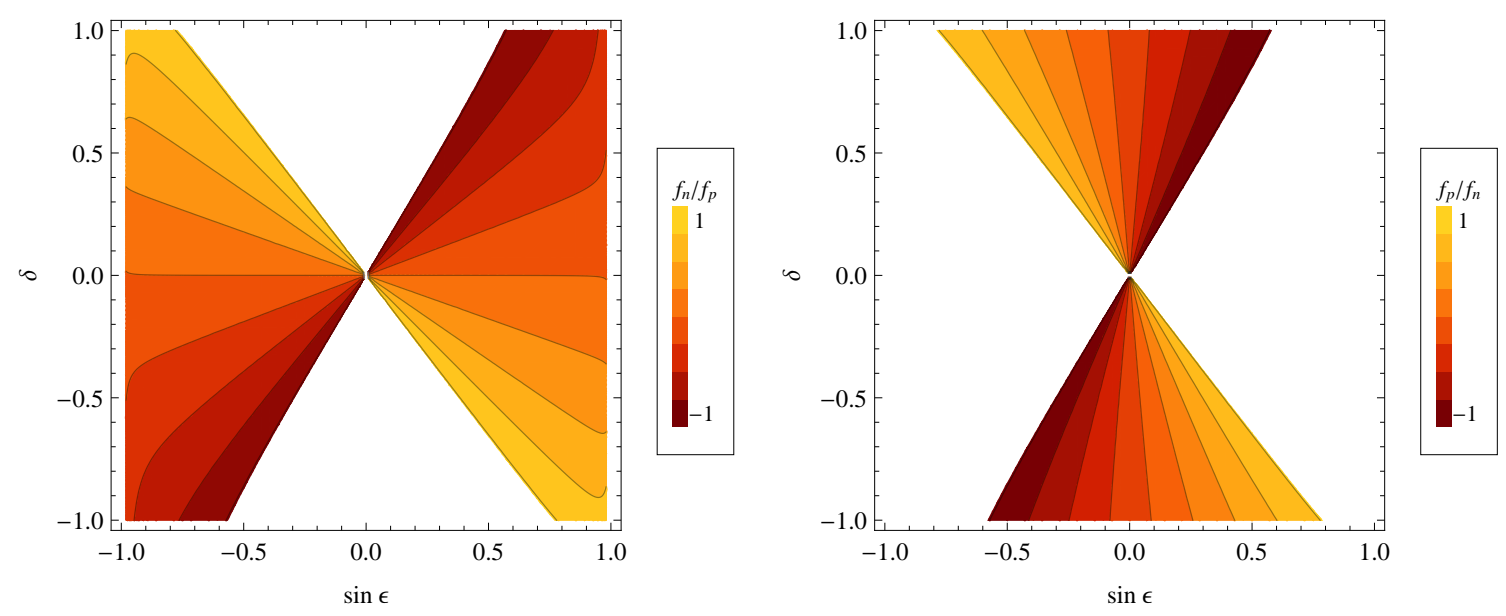

Figure 2.14 Contour plots of $f_{n} / f_{p}$ and $f_{p} / f_{n}$ as a function of $\epsilon$ and $\delta$. No limits are placed on the values of $\epsilon$ and $\delta$.

Finally, we can write the dark matter-nucleus spin-independent scattering cross section:

$$
\sigma_{A}=\frac{\mu_{A}^{2}}{\mu_{p}^{2}}\left(Z+(A-Z) \frac{f_{n}}{f_{p}}\right)^{2} \sigma_{p},
$$

where $\sigma_{p}$ is the spin-independent DM-proton cross section,

$$
\sigma_{p}=\frac{\mu_{p}^{2} f_{p}^{2}}{64 \pi},
$$

and $Z$ and $A$ are the atomic and mass numbers of the detector material, $\mu_{A}$ is the reduced mass of the dark matter-nucleus system, and $\mu_{p}$ is the reduced mass of the dark matter-proton system [11].

It is common to present the spin-independent cross section for a nucleus with $\mathrm{Z}$ protons in terms of the cross section for scattering off a single nucleon, making the 
assumption that $f_{n}=f_{p}$. To compare a model with data, we must also account for possible isospin violation. To convert the nuclear cross section to a proton cross section $\sigma_{p}$, we multiply by 11

$$
F_{Z}=\frac{\sum_{i} \eta_{i} \mu_{A_{i}}^{2} A_{i}^{2}}{\sum_{i} \eta_{i} \mu_{A_{i}}^{2}\left[Z+\left(A_{i}-Z\right) f_{n} / f_{p}\right]^{2}},
$$

This factor is derived from Eq. (2.39) by summing over all stable isotopes of atomic number $Z$, weighted by their natural abundances $\eta_{i}$. In Fig. 2.15, we show the effect of isospin violation on the xenon $(\mathrm{Z}=54)$ cross section, normalized to the current limit from XENON100, $\sigma_{p}<2.7 \times 10^{-45} \mathrm{~cm}^{2}$ at $f_{n} / f_{p}=1$ and $M_{\chi}=100 \mathrm{GeV}$ [27]. Isospin violation can relax the bound by several orders of magnitude, with the least stringent bound occurring around $f_{n} / f_{p}=-0.7[11$.

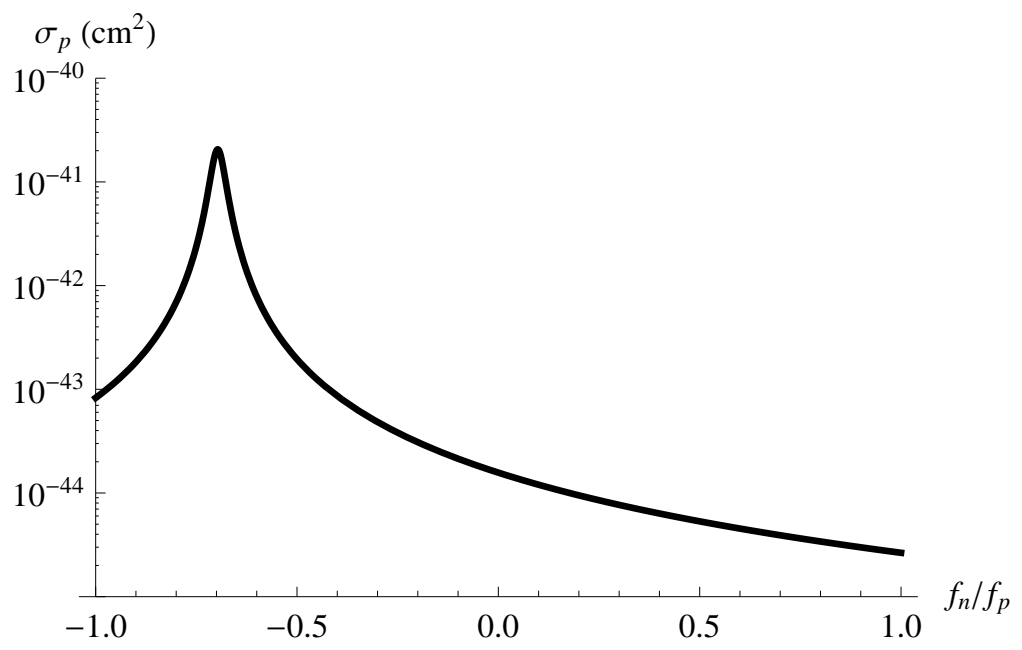

Figure 2.15 The $90 \%$ C.L. upper bound on the spin independent dark matter-proton scattering cross section from XENON100 as a function of $f_{n} / f_{p}$.

With the XENON bound generalized to all values of $f_{n} / f_{p}$, we can use it to place limits on the model parameters. We start by choosing a random sample of points $(\epsilon, \xi)$ within the allowed region shown in Fig. 2.13 and then calculate the proton SI cross section for each point. In Figs. 2.16 and 2.17, we show the dependence of the cross section on $f_{n} / f_{p}$ and $M_{Z^{\prime}}$, respectively. From Fig. 2.17, we see that the largest cross 


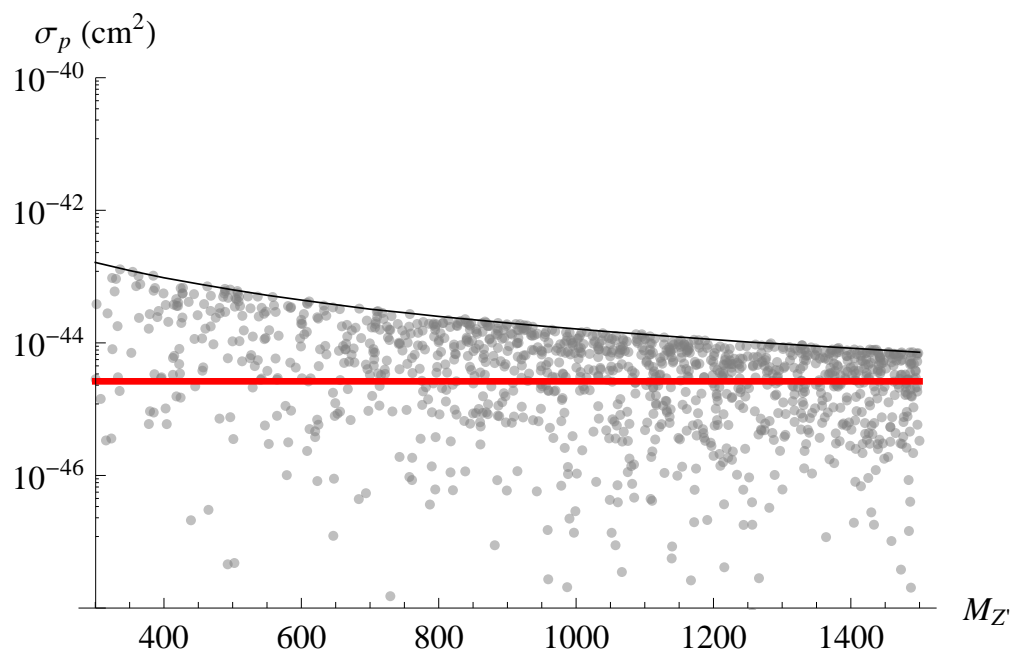

Figure 2.16 The cross section fro DM scattering on protons, $\sigma_{p}$ versus $Z^{\prime}$ mass for $f_{n} / f_{p}=1$. The thick red line at $\sigma_{p}=2.7 \times 10^{-45} \mathrm{~cm}^{2}$ is the XENON100 limit [27]; dots are the cross sections corresponding to pairs $(\epsilon, \delta)$ sampled uniformly over the allowed values. The dark matter mass and coupling to the $Z^{\prime}$ are set to $M_{\chi}=100 \mathrm{GeV}$ and $g_{\chi}=1$, respectively.

sections occur for $f_{n} / f_{p} \approx 0.35$, while the lowest occur for $f_{n} / f_{p}<-0.5$, which is where the XENON bound is the most relaxed. For both figures, we have set $g_{\chi}=1$ and $M_{\chi}=100 \mathrm{GeV}$, which in general are free parameters. However, they have no impact on the qualitative features of the distributions, as they only enter the overall normalization factor. They influence our ability to place limits on the other parameters; in particular, the $g_{\chi}^{2}$ dependence of the cross section means that all constraints can be evaded by choosing a small enough coupling. For $M_{Z^{\prime}}=1000 \mathrm{GeV}$ and $M_{\chi}=100 \mathrm{GeV}$, the XENON bounds are evaded for all values of $f_{n} / f_{p}$ with $g_{\chi}=0.58$.

Collider searches can also help constrain the mixing angles for a dark $Z^{\prime}$, as the $Z^{\prime}$ acquires small couplings to SM fermions via mixing effects [73]. $Z^{\prime}$ production and decay to leptons at the LHC depends primarily on the mixing angles and the $Z^{\prime}$ mass, with only a small dependence on the dark matter properties through the decay width of the $Z^{\prime}$. Using the couplings in Eq. (2.32), we can apply the analysis of Section 2.4 to calculate the cross section for $Z^{\prime}$ production at the LHC. We find the ATLAS predictions of the 


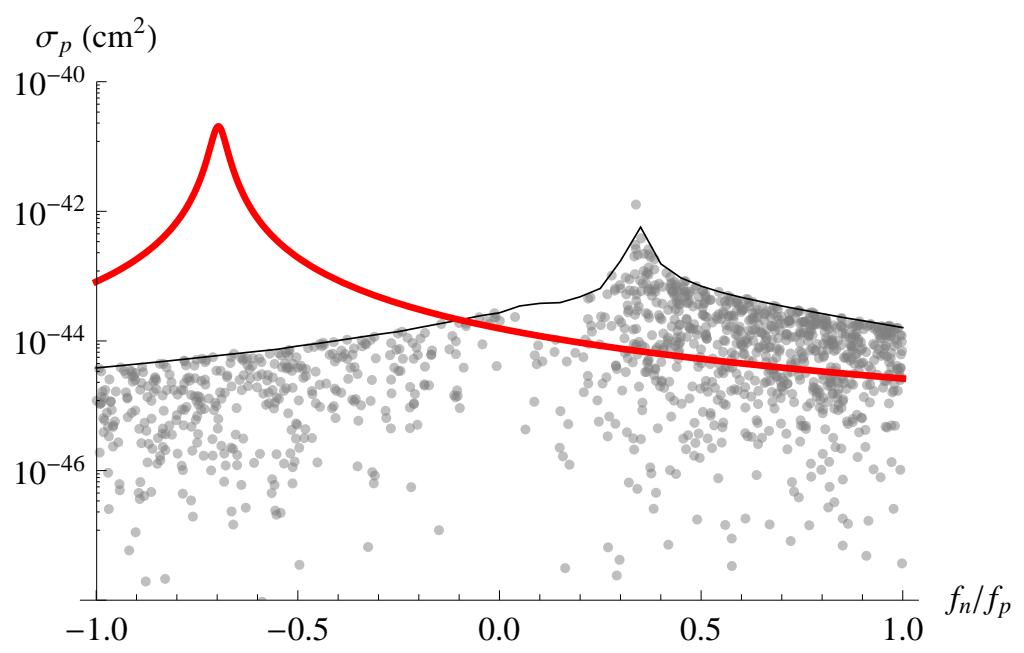

Figure $2.17 \sigma_{p}$ versus $f_{n} / f_{p}$ mass for $M_{Z}^{\prime}=1000 \mathrm{GeV}$. The thick red line is the XENON100 upper bound; dots are the cross sections corresponding to pairs $(\epsilon, \delta)$ sampled uniformly through the allowed region shown in Fig 2.13. The dark matter mass and coupling to the $Z^{\prime}$ are set to $M_{\chi}=100 \mathrm{GeV}$ and $g_{\chi}=1$, respectively.

cross section for the various $Z^{\prime}$ models given in Fig. 2 of Ref. [25] are well parameterized by

$$
\sigma B=(2200 \mathrm{fb}) r_{Z^{\prime}}^{12}\left(\frac{1}{r_{Z^{\prime}}}-1\right)^{15}\left[p c_{u}+(1-p) c_{d}\right] .
$$

With this equation, we can use the current ATLAS limits to restrict the parameter space. Since there are no direct couplings between the $Z^{\prime}$ and the SM, the lower bound on the $Z^{\prime}$ mass is much less stringent than for the models considered earlier. In Fig. 2.18, we show the upper bound on $\sigma\left(p p \rightarrow Z^{\prime} \rightarrow l^{+} l^{-}\right)$set by the $S$ and $T$ parameters for $\sqrt{s}=7 \mathrm{TeV}$.

Finally, we can combine the limits from XENON100 and ATLAS to constrain the model parameter space in terms of $\epsilon, \delta$, and $M_{Z^{\prime}}$. The results are shown in Fig. 3.7, again with $M_{\chi}=100 \mathrm{GeV}$ and $g_{\chi}=1$. The electroweak, dark matter, and LHC data provide complimentary bounds, with $S$ and $T$ more strongly limiting the degree of mass mixing, while XENON and ATLAS provide more stringent bounds on kinetic mixing. The bounds on $\delta$ and $\epsilon$ relax as $M_{Z^{\prime}}$ increases and the $Z$ and $Z^{\prime}$ decouple. Note that 


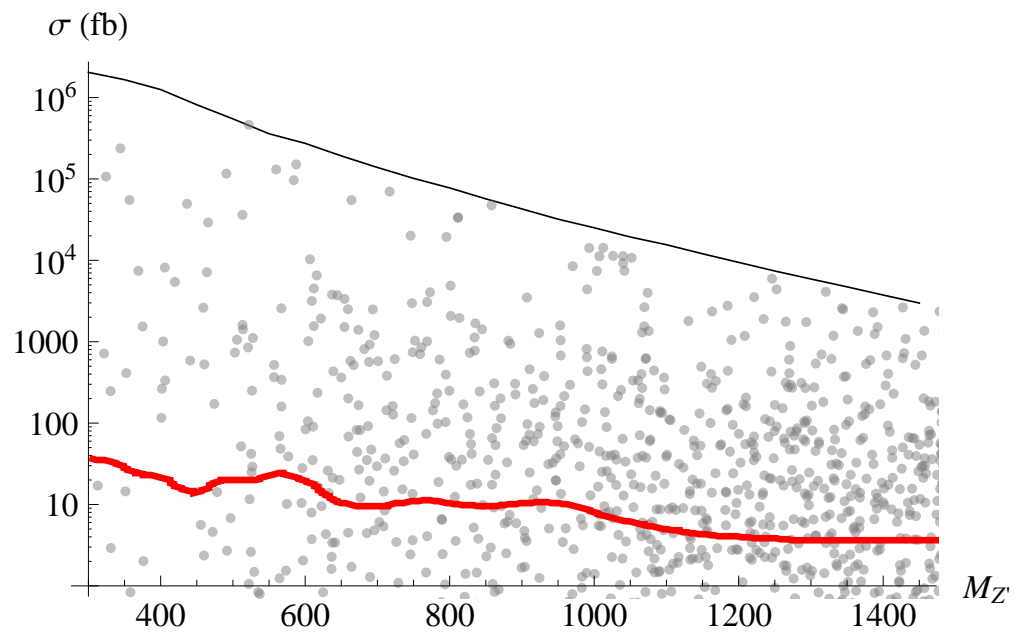

Figure $2.18 \sigma\left(p p \rightarrow Z^{\prime} \rightarrow l^{+} l^{-}\right)$versus $M_{Z}^{\prime}$. The thick red line is the most recent ATLAS limit 25]; dots are the cross sections corresponding to pairs $(\epsilon, \delta)$ sampled uniformly through the allowed region shown in Fig 2.13.

the experiments report their results at different confidence levels, so these regions are not confidence regions; they are simply indicative of the parameter space available.

The invisible decay width of the $Z$ and the muon anomalous magnetic moment have also been used to constrain the mixing parameters $\epsilon$ and $\delta$ [67], but they are less restrictive than $S$ and $T$ for $M_{Z^{\prime}}$ in the range $300-1500 \mathrm{GeV}$.

If $M_{Z}>M_{\chi}$, the invisible decay width of the $Z$ must be considered. The decay width is proportional to $\xi^{2}$, so the experimental $1.5 \mathrm{MeV}$ bound [57] is avoided as long as $\xi<0.95$.

There are also corrections to the muon anomalous magnetic moment [67],

$$
\delta a_{\mu} \approx \frac{\alpha \xi^{2}}{3 \pi c_{W}^{2} s_{W}^{2}} \frac{m_{\mu}^{2}}{M_{Z^{\prime}}^{2}} .
$$

However, the contributions from the $Z^{\prime}$ are very small, because of the dependence on $M_{Z^{\prime}}^{-2}$ and $\xi^{2}$. The current experimental limit is $\delta a_{\mu} \approx 3 \times 10^{-9}$ [57], which is several orders of magnitude larger than the expected contribution except for very small $M_{Z^{\prime}}$. 

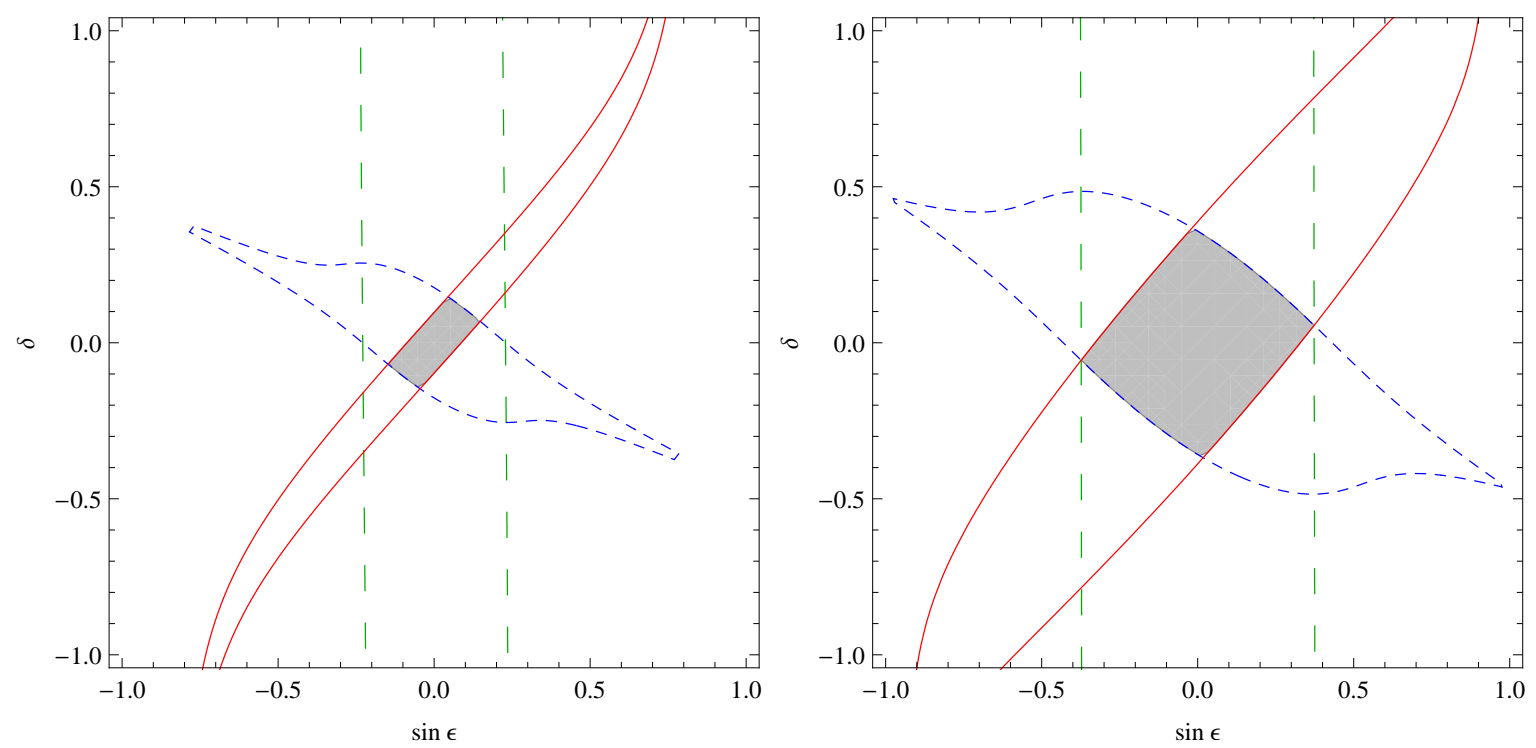

Figure 2.19 Derived limits on $\epsilon$ and $\delta$ for $M_{Z^{\prime}}=500 \mathrm{GeV}$ (left) and $M_{Z^{\prime}}=1000 \mathrm{GeV}$ (right). The dotted blue lines represent the limit from global electroweak data (90\% C.L.), the solid red lines are the limit from XENON100 (90\% C.L.), and the dashed green lines are the limits from ATLAS at $\sqrt{s}=7 \mathrm{TeV}$ for $\mathcal{L} \approx 1 \mathrm{fb}^{-1}$ (95\% C.L.).

\section{7 $\quad$ Summary of $Z^{\prime}$ Analysis}

Our customization of the FEWZ simulation code allows for extensive studies of $Z^{\prime}$ production and decay at the LHC at NLO and NNLO. Using the results of our simulation for representative benchmark models, we derived an empirical formula for the double differential cross section $\frac{d^{2} \sigma}{d y d \cos \theta}$. This formula can be used to study broad classes of models easily and to determine the $Z^{\prime}$ couplings to fermions without prior knowledge of the underlying model. In a model-dependent analysis, it can also be used to set limits on model parameters. In the case of the $E_{6}$-derived models, without accounting for systematic uncertainties, we find that the mixing angle $\beta$ may be determined within $0.1 \pi$ with $1 \mathrm{ab}^{-1}$ of data for a mass of $2.5 \mathrm{TeV}$. For $E_{6}$ models we also showed that within a range of mixing angles, two extra neutral gauge bosons should be within the reach of the LHC.

Finally, we considered a more general $Z^{\prime}$ model with kinetic and mass mixing, which has interesting implications for dark matter detection and hidden sector theories. Even 
if the SM is uncharged under a new hidden sector $U(1)^{\prime}$, mixing could induce couplings strong enough that the $Z^{\prime}$ could be produced at the LHC and mediate dark matter scattering on nuclei, without violating limits from global electroweak data. In the case of a heavy $Z^{\prime}\left(M_{Z^{\prime}} \gg M_{Z}\right)$ we find that the limits on the $S$ and $T$ parameters can be combined with XENON100 and LHC data to restrict the range of allowed mixing angles. For a relatively light $Z^{\prime}$ at $500 \mathrm{GeV}$, the mass and kinetic mixing parameters $\delta$ and $\epsilon$ must both be less than about 0.2 . For heavy $Z^{\prime}$ s, these mixing parameters are unrestricted. 


\section{Chapter 3}

\section{$W^{\prime}$ decays to odd Higgs particles}

\subsection{Introduction}

New heavy particles of charge \pm 1 and spin 1 , referred to as $W^{\prime}$ bosons, are predicted in many interesting theories for physics beyond the Standard Model (SM) [2, 13]. Extensive searches for $W^{\prime}$ bosons at colliders have set limits on the production cross section times branching fraction in several final states [2]. The most stringent limit on a $W^{\prime}$ boson that has the same couplings to quarks and leptons as the SM $W$ boson ("sequential" $W^{\prime}$ ) has been set using the $\ell \nu$ channels, where $\ell=e$ or $\mu$; the current mass limit is $3.8 \mathrm{TeV}$, set by the CMS Collaboration [20] using the full data set from the $8 \mathrm{TeV}$ LHC.

In this Chapter, we show that the $W^{\prime}$ boson is likely to decay not only into SM fermions, as often assumed, but also into pairs of scalar particles from the extended Higgs sector responsible for the $W^{\prime}$ mass. As a result the existing limits may be relaxed, and different types of searches at the LHC may prove to be more sensitive.

Theories that include a $W^{\prime}$ boson embed the electroweak gauge group within an $S U(2)_{1} \times S U(2)_{2} \times U(1), S U(3)_{W} \times U(1)$, or larger gauge symmetry that is spontaneously broken down to the electromagnetic gauge group, $U(1)_{\mathrm{em}}$. This symmetry breaking pattern is induced usually by some scalar fields with vacuum expectation values (VEVs). The coupling of the $W^{\prime}$ to these scalars is related to the gauge couplings, and cannot be too small. In perturbative renormalizable models, the scalars have masses near or below the symmetry breaking scale, because the quartic couplings grow with the energy. The 
$W^{\prime}$ boson, by contrast, may be significantly heavier, because large gauge couplings are allowed by the asymptotic freedom of non-Abelian gauge theories. Consequently, it is natural to expect $W^{\prime}$ decays into pairs of particles from the extended Higgs sector.

We demonstrate the importance of $W^{\prime}$ decays into scalars by analyzing in detail a simple renormalizable $W^{\prime}$ model: $S U(2)_{1} \times S U(2)_{2} \times U(1)_{Y}$ gauge symmetry broken by the VEVs of two complex scalars: a bidoublet (i.e., a doublet under each non-Abelian group) of hypercharge $Y=0$ and a doublet under one of the $S U(2)$ 's. This model has been studied in different contexts [74, 75], assuming that the Higgs particles are heavy enough to avoid $W^{\prime}$ decays into them. An interesting feature of it is that, up to an overall normalization, the $W^{\prime}$ boson has identical couplings to quarks and leptons as the SM $W$ boson. We refer to it as the "meta-sequential" $W^{\prime}$.

The most general scalar potential has many terms, but it is significantly simplified by imposing a $\mathbb{Z}_{2}$ symmetry (the bidoublet transforms into its charge conjugate). The lightest Higgs particle that is odd under this parity is stable, and could be a viable dark matter candidate. Whether or not the $\mathbb{Z}_{2}$ is exact, it leads to cascade decays of the $W^{\prime}$ that give signatures with one or two electroweak bosons and two of these lightest odd particles (LOPs).

In Section 3.2 we study the masses and couplings of the Higgs particles, and of the heavy gauge bosons. Then, in Section 3.3, we compute the branching fractions of the $W^{\prime}$ and $Z^{\prime}$ bosons, and comment on various signatures arising from their cascade decays. In Section 3.4 we discuss the LHC phenomenology assuming that the LOPs escape the detector. We summarize our results in Section 3.5.

\subsection{An $S U(2) \times S U(2) \times U(1)_{Y}$ model with odd Higgs sector}

Let us focus on a simple Higgs sector that breaks the $S U(2)_{1} \times S U(2)_{2} \times U(1)_{Y}$ gauge group down to $U(1)_{\mathrm{em}}$ : a bidoublet complex scalar, $\Delta$, which has 0 hypercharge, and an $S U(2)_{1}$ doublet, $\Phi$. We take the SM quarks and leptons to be $S U(2)_{2}$ singlets. The scalar and fermion gauge charges are shown in Table 3.1. 


\begin{tabular}{|c||c|c|c|c|}
\hline & $S U(3)_{c}$ & $S U(2)_{1}$ & $S U(2)_{2}$ & $U(1)_{Y}$ \\
\hline \hline$\Delta$ & 1 & 2 & $\overline{2}$ & 0 \\
\hline$\Phi$ & 1 & 2 & 1 & $+\frac{1}{2}$ \\
\hline$Q_{L}, L_{L}$ & 3,1 & 2 & 1 & $+\frac{1}{6},-\frac{1}{2}$ \\
\hline$u_{R}, d_{R}$ & 3 & 1 & 1 & $+\frac{2}{3},-\frac{1}{3}$ \\
\hline$e_{R}$ & 1 & 1 & 1 & +1 \\
\hline
\end{tabular}

Table 3.1 Gauge assignments for the scalars $(\Delta$ and $\Phi)$ and SM fermions.

\subsubsection{Scalar spectrum}

We require the Lagrangian to be symmetric under the interchange $\Delta \leftrightarrow \tilde{\Delta}$, where $\tilde{\Delta}$ is the charge conjugate of $\Delta$. The most general renormalizable scalar potential exhibiting this $\mathbb{Z}_{2}$ symmetry and CP invariance is [74]

$$
\begin{aligned}
V= & m_{\Phi}^{2} \Phi^{\dagger} \Phi+\frac{\lambda_{\Phi}}{2}\left(\Phi^{\dagger} \Phi\right)^{2}+\left(m_{\Delta}^{2}+\lambda_{0} \Phi^{\dagger} \Phi\right) \operatorname{Tr}\left(\Delta^{\dagger} \Delta\right)+\frac{\lambda_{\Delta}}{2}\left[\operatorname{Tr}\left(\Delta^{\dagger} \Delta\right)\right]^{2} \\
& -\frac{\tilde{\lambda}}{2}\left|\operatorname{Tr}\left(\Delta^{\dagger} \tilde{\Delta}\right)\right|^{2}-\left[\frac{\tilde{\lambda}^{\prime}}{4}\left(\operatorname{Tr}\left(\Delta^{\dagger} \tilde{\Delta}\right)\right)^{2}+\text { H.c. }\right] .
\end{aligned}
$$

To avoid runaway directions, we impose $\lambda_{\Phi}, \lambda_{\Delta}>0$. The $\tilde{\lambda}$ and $\lambda_{0}$ quartic couplings must be real so that the potential is Hermitian. The $\tilde{\lambda}^{\prime}$ quartic coupling may be complex, but its phase can be rotated away by a redefinition of $\Delta$; we then take $\tilde{\lambda}^{\prime}$ to be real without loss of generality.

Canonical normalization of the $\tilde{\lambda}$ and $\tilde{\lambda}^{\prime}$ terms would require an extra factor of $1 / 2$; we do not include it in order to simplify some equations below. Other terms in $V$, such as $\operatorname{Tr}\left[\left(\Delta^{\dagger} \Delta\right)^{2}\right], \operatorname{Tr}\left(\Delta^{\dagger} \Delta \tilde{\Delta}^{\dagger} \tilde{\Delta}\right)$, or $\operatorname{Tr}\left(\Delta^{\dagger} \tilde{\Delta} \tilde{\Delta}^{\dagger} \Delta\right)$, would be redundant as they are linear combinations of the $\lambda_{\Delta}, \tilde{\lambda}$ and $\tilde{\lambda}^{\prime}$ terms. We recover the potential of Ref. [74] using the identity $\Phi^{\dagger}\left(\Delta^{\dagger} \Delta+\tilde{\Delta}^{\dagger} \tilde{\Delta}\right) \Phi=\Phi^{\dagger} \Phi \operatorname{Tr}\left(\Delta^{\dagger} \Delta\right)$. 
We also impose $m_{\Delta}^{2}<0$ so that $\Delta$ acquires a VEV. In addition, we need $m_{\Phi}^{2}<0$ or $\lambda_{0}<0$ such that $\Phi$ also acquires a VEV. We are interested in the vacuum that preserves the $U(1)_{\mathrm{em}}$ and $\mathbb{Z}_{2}$ symmetries:

$$
\langle\Delta\rangle=\frac{v_{\Delta}}{2} \operatorname{diag}(1,1) \quad, \quad\langle\Phi\rangle=\frac{v_{\phi}}{\sqrt{2}}\left(\begin{array}{l}
0 \\
1
\end{array}\right) .
$$

This vacuum is indeed a minimum of the potential for a range of parameters (discussed below). The VEVs $v_{\phi}>0$ and $v_{\Delta}>0$ are related to $m_{\Phi}^{2}, m_{\Delta}^{2}$, and the five quartic couplings by the extremization conditions:

$$
\begin{aligned}
& \lambda_{\star} v_{\Delta}^{2}+\lambda_{0} v_{\phi}^{2}=-2 m_{\Delta}^{2}, \\
& \lambda_{0} v_{\Delta}^{2}+\lambda_{\Phi} v_{\phi}^{2}=-2 m_{\Phi}^{2}
\end{aligned}
$$

where we defined

$$
\lambda_{\star} \equiv \lambda_{\Delta}-\tilde{\lambda}-\tilde{\lambda}^{\prime}
$$

In terms of fields of definite electric charge, the scalars can be written as

$$
\begin{aligned}
\Phi & =\left(\begin{array}{c}
\phi^{+} \\
\frac{1}{\sqrt{2}}\left(v_{\phi}+\phi_{r}^{0}+i \phi_{i}^{0}\right)
\end{array}\right), \\
\Delta & =\left(\begin{array}{cc}
\eta^{0} & \chi^{+} \\
\eta^{-} & \chi^{0}
\end{array}\right)=\langle\Delta\rangle+\left(\begin{array}{cc}
\frac{1}{\sqrt{2}}\left(\eta_{r}^{0}+i \eta_{i}^{0}\right) & \chi^{+} \\
\eta^{-} & \frac{1}{\sqrt{2}}\left(\chi_{r}^{0}+i \chi_{i}^{0}\right)
\end{array}\right) .
\end{aligned}
$$

The charge conjugate state of the bidoublet is then

$$
\tilde{\Delta}=\sigma_{2} \Delta^{*} \sigma_{2}=\left(\begin{array}{cc}
\chi^{0 *} & -\eta^{+} \\
-\chi^{-} & \eta^{0 *}
\end{array}\right)
$$

All odd fields under $\mathbb{Z}_{2}$ (which cannot mix with even fields, and thus are already in the mass eigenstate basis) are collected in

$$
\Delta-\tilde{\Delta}=\left(\begin{array}{cc}
H^{0}+i A^{0} & \sqrt{2} H^{+} \\
\sqrt{2} H^{-} & -H^{0}+i A^{0}
\end{array}\right),
$$


where the physical states consist of a CP-even scalar $\left(H^{0}\right)$, a CP-odd scalar $\left(A^{0}\right)$, and a charged scalar $\left(H^{ \pm}\right)$. These are related to the $\eta$ and $\chi$ fields by

$$
\begin{aligned}
& A^{0}=\frac{1}{\sqrt{2}}\left(\eta_{i}^{0}+\chi_{i}^{0}\right), \\
& H^{0}=\frac{1}{\sqrt{2}}\left(\eta_{r}^{0}-\chi_{r}^{0}\right), \\
& H^{ \pm}=\frac{1}{\sqrt{2}}\left(\eta^{ \pm}+\chi^{ \pm}\right)
\end{aligned}
$$

At tree-level, the $\mathbb{Z}_{2}$-odd scalars have masses given by

$$
\begin{aligned}
& M_{A}=\sqrt{2 \tilde{\lambda}^{\prime}} v_{\Delta}, \\
& M_{H^{+}}=M_{H^{0}}=\sqrt{\tilde{\lambda}+\tilde{\lambda}^{\prime}} v_{\Delta} .
\end{aligned}
$$

There are two remaining scalars not eaten by the gauge bosons. These are $\mathbb{Z}_{2}$-even, CP-even, and neutral; their mass-squared matrix in the $\left(\chi_{r}^{0}+\eta_{r}^{0}\right) / \sqrt{2}, \phi_{r}^{0}$ basis is

$$
\mathcal{M}_{\text {even }}^{2}=\left(\begin{array}{cc}
\lambda_{\star} v_{\Delta}^{2} & \lambda_{0} v_{\phi} v_{\Delta} \\
\lambda_{0} v_{\phi} v_{\Delta} & \lambda_{\Phi} v_{\phi}^{2}
\end{array}\right) \text {. }
$$

The $\mathbb{Z}_{2}$-even physical scalars,

$$
\begin{aligned}
h^{0} & =\phi_{r}^{0} \cos \alpha_{h}-\frac{1}{\sqrt{2}}\left(\chi_{r}^{0}+\eta_{r}^{0}\right) \sin \alpha_{h}, \\
H^{\prime 0} & =\phi_{r}^{0} \sin \alpha_{h}+\frac{1}{\sqrt{2}}\left(\chi_{r}^{0}+\eta_{r}^{0}\right) \cos \alpha_{h},
\end{aligned}
$$

have the following squared masses:

$$
M_{h, H^{\prime}}^{2}=\frac{1}{2}\left(\lambda_{\star} v_{\Delta}^{2}+\lambda_{\Phi} v_{\phi}^{2} \mp \sqrt{\left(\lambda_{\star} v_{\Delta}^{2}-\lambda_{\Phi} v_{\phi}^{2}\right)^{2}+4 \lambda_{0}^{2} v_{\phi}^{2} v_{\Delta}^{2}}\right) .
$$

The mixing angle $\alpha_{h}$ satisfies

$$
\tan 2 \alpha_{h}=\frac{2 \lambda_{0} v_{\Delta} v_{\phi}}{\lambda_{\star} v_{\Delta}^{2}-\lambda_{\Phi} v_{\phi}^{2}}
$$


The necessary and sufficient conditions for the vacuum $(3.2)$ to be a minimum of the potential are

$$
\begin{aligned}
\tilde{\lambda}^{\prime} & >\operatorname{Max}\{-\tilde{\lambda}, 0\}, \\
\lambda_{\star} \lambda_{\Phi} & >\lambda_{0}^{2} \\
\lambda_{\Phi}\left|m_{\Delta}^{2}\right| & >-\lambda_{0} m_{\Phi}^{2} \\
\lambda_{0}\left|m_{\Delta}^{2}\right| & >-\lambda_{\star} m_{\Phi}^{2}
\end{aligned}
$$

these follow from imposing that all physical scalars have positive squared masses [see Eqs. (3.9) and (3.12)], and that the extremization conditions 3.3 have solutions.

All above results are valid for any $v_{\phi} / v_{\Delta}$. The agreement between SM predictions and the data suggests that the Higgs sector is near the decoupling limit $v_{\phi}^{2} \ll v_{\Delta}^{2}$; adopting this limit, we can analyze the spontaneous symmetry breaking in two stages. The first one is $S U(2)_{1} \times S U(2)_{2} \times U(1)_{Y} \rightarrow S U(2)_{W} \times U(1)_{Y}$ at the scale $v_{\Delta}$. The effective theory below $v_{\Delta}$ consists of the SM (with the Higgs doublet $\Phi$ ) plus an $S U(2)_{W}$-triplet of heavy gauge bosons $\left(W^{\prime \pm}, Z^{\prime}\right)$, and five of the scalar degrees of freedom from $\Delta$ : four

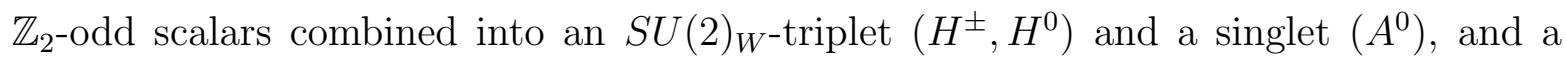
$\mathbb{Z}_{2}$-even singlet $\left(H^{\prime 0}\right)$.

The second stage of symmetry breaking is the SM one: $S U(2)_{W} \times U(1)_{Y} \rightarrow U(1)_{\mathrm{em}}$ at the weak scale $v_{\phi} \approx 246 \mathrm{GeV}$. The lightest CP-even scalar, $h^{0}$, represents the recently discovered Higgs boson, because its couplings are the same as the SM ones up to small corrections of order $v_{\phi}^{2} / v_{\Delta}^{2}$. Its mass is given by

$$
M_{h}=v_{\phi}\left(\lambda_{\Phi}-\frac{\lambda_{0}^{2}}{\lambda_{\star}}\right)^{1 / 2}\left[1-\frac{\lambda_{0}^{2} v_{\phi}^{2}}{2 \lambda_{\star}^{2} v_{\Delta}^{2}}+O\left(v_{\phi}^{4} / v_{\Delta}^{4}\right)\right],
$$

and should be identified with the measured Higgs mass, near $126 \mathrm{GeV}$. The $H^{\prime 0}$ even scalar has the same couplings as the SM Higgs except for an overall suppression by

$$
\sin \alpha_{h}=\frac{\lambda_{0} v_{\phi}}{\lambda_{\star} v_{\Delta}}+O\left(v_{\phi}^{3} / v_{\Delta}^{3}\right)
$$


and is significantly heavier:

$$
M_{H^{\prime}}=\sqrt{\lambda_{\star}} v_{\Delta}+O\left(v_{\phi}^{2} / v_{\Delta}\right)
$$

Consequently, its dominant decay modes are $W^{+} W^{-}$and $Z Z$.

The odd scalars, $H^{ \pm}, H^{0}, A^{0}$, couple exclusively to gauge bosons and scalars, and only in pairs. The lightest of them is stable, and a component of dark matter. $A^{0}$ is naturally the lightest odd particle (LOP) because in the $\tilde{\lambda}^{\prime} \rightarrow 0$ limit the symmetry is enhanced: $A^{0}$ becomes the Nambu-Goldstone boson of a global $U(1)$ symmetry acting on $\Delta$. We note, however, that $H^{0}$ could also be the LOP (for $\tilde{\lambda}^{\prime}>\tilde{\lambda}$ ) and a viable dark matter candidate. Even though it is part of an $S U(2)_{W}$ triplet that is degenerate at tree-level, electroweak loops split the $H^{ \pm}$and $H^{0}$ masses [76, 77].

In what follows we will assume that $A^{0}$ is the LOP. The heavier odd scalars then decay as follows: $H^{ \pm} \rightarrow W^{ \pm} A^{0}, H^{0} \rightarrow Z A^{0}$. Even when these two-body decays are kinematically forbidden, the three-body decays through an off-shell $W^{ \pm}$or $Z$ are the dominant ones. Other channels are highly suppressed, either kinematically $\left(H^{+} \rightarrow\right.$ $\pi^{+} \pi^{0} H^{0}$ and $\left.H^{+} \rightarrow \ell^{+} \nu H^{0}\right)$ or by loops $\left(H^{0} \rightarrow \gamma A^{0}\right.$ and the CP-violating $\left.H^{0} \rightarrow h^{0} A^{0}\right)$.

\subsubsection{Meta-sequential $W^{\prime}$ boson}

The kinetic terms for the $\Phi$ and $\Delta$ scalars,

$$
\left(D_{\mu} \Phi\right)^{\dagger} D_{\mu} \Phi+\operatorname{Tr}\left[\left(D_{\mu} \Delta\right)^{\dagger} D_{\mu} \Delta\right]
$$

involve the covariant derivative

$$
D_{\mu}=\partial_{\mu}-i g_{Y} Y B_{\mu}-i g_{1} \vec{T}_{1} \cdot \vec{W}_{1 \mu}-i g_{2} \vec{T}_{2} \cdot \vec{W}_{2 \mu}
$$

with $T_{1,2}=\sigma_{1,2} / 2$; notice that $\vec{T}_{2}$ acts from the right on the bidoublet: $\vec{T}_{2} \cdot \Delta=-\Delta \cdot \vec{\sigma} / 2$. After symmetry breaking, the electrically-charged gauge bosons acquire mass terms:

$$
\frac{v_{\phi}^{2}}{4} g_{1}^{2} W_{1 \mu}^{+} W_{1}^{-\mu}+\frac{v_{\Delta}^{2}}{4}\left(g_{1} W_{1 \mu}^{+}-g_{2} W_{2 \mu}^{+}\right)\left(g_{1} W_{1}^{-\mu}-g_{2} W_{2}^{-\mu}\right) .
$$


Diagonalizing them gives the physical charged spin-1 states,

$$
\begin{aligned}
& W_{\mu}=W_{1 \mu} \cos \theta+W_{2 \mu} \sin \theta \\
& W_{\mu}^{\prime}=-W_{1 \mu} \sin \theta+W_{2 \mu} \cos \theta
\end{aligned}
$$

with the following mixing angle, $0 \leq \theta \leq \pi / 2$ :

$$
\tan 2 \theta=\frac{2 g_{1} g_{2} v_{\Delta}^{2}}{\left(g_{2}^{2}-g_{1}^{2}\right) v_{\Delta}^{2}-g_{1}^{2} v_{\phi}^{2}} .
$$

The masses of the $W$ and $W^{\prime}$ bosons are

$$
M_{W, W^{\prime}}=\frac{1}{2 \sqrt{2}}\left[\left(g_{2}^{2}+g_{1}^{2} \mp \frac{2 g_{1} g_{2}}{\sin 2 \theta}\right) v_{\Delta}^{2}+g_{1}^{2} v_{\phi}^{2}\right]^{1 / 2} \text {. }
$$

Given that the left-handed quarks and leptons transform as doublets only under $S U(2)_{1}$, their couplings to the $W$ and $W^{\prime}$ bosons are proportional to the respective coefficients of $W_{1 \mu}$ in Eqs. (3.21). The measured $W$ coupling to fermions gives a value for the $S U(2)_{W}$ gauge coupling of $g=\sqrt{4 \pi \alpha} / s_{W} \approx 0.652$, where the electromagnetic coupling constant and the weak mixing angle are evaluated at the $M_{Z}$ scale: $\alpha \equiv$ $\alpha\left(M_{Z}\right) \approx 1 / 127.9$ and $s_{W} \equiv \sin \theta_{W} \approx \sqrt{0.231}$. In terms of the parameters of this model, the $S U(2)_{W}$ gauge coupling can be expressed as

$$
g_{1} \cos \theta=g
$$

The $W^{\prime}$ coupling to quarks and leptons, derived from Eq. (3.21) and Table I, is then

$$
-g_{1} \sin \theta=-g \tan \theta
$$

Thus, $\tan \theta$ determines completely the tree-level couplings of $W^{\prime}$ to SM fermions. Imposing a perturbativity condition on the $S U(2)_{1} \times S U(2)_{2}$ gauge couplings, $g_{1,2}^{2} /(4 \pi) \lesssim 1$, and using Eq. (3.24) we find that

$$
0.2 \lesssim \tan \theta \lesssim 5
$$

In the particular case of $\tan \theta=1$, the couplings of $W^{\prime}$ to fermions are identical (at tree level) to those of the $W$. This is usually referred to as the sequential $W^{\prime}$ boson, and 
is a common benchmark model for $W^{\prime}$ searches at colliders. The most recent limit on the mass of a sequential $W^{\prime}$ at CMS, using $20 \mathrm{fb}^{-1}$ of $8 \mathrm{TeV}$ data, is $3.8 \mathrm{TeV}$ [20], assuming that $W^{\prime}$ can decay only into SM fermions. Note that the relative sign in Eqs. (3.24) and 3.25 implies constructive interference between the $W$ and $W^{\prime}$ amplitudes that contribute to processes constrained by $W^{\prime}$ searches at the LHC. In the next sections we will focus on the region $0.2<\tan \theta<1$, where the LHC limits are relaxed. Given that the $W^{\prime}$ boson in this model has couplings to fermions proportional to the SM $W$ ones (by an overall factor of $-\tan \theta$ ), we refer to it as a "meta-sequential $W^{\prime}$ boson".

The above results are valid for any $v_{\phi} / v_{\Delta}$. It is instructive to expand these results in powers of $\left(v_{\phi} / v_{\Delta}\right)^{2} \ll 1$. The $W^{\prime}$ coupling to fermions, relative to the $W$ one is

$$
\tan \theta=\tan \theta_{0}\left(1-\frac{v_{\phi}^{2}}{v_{\Delta}^{2}} \cos ^{2} \theta_{0}\right)+O\left(v_{\phi}^{4} / v_{\Delta}^{4}\right)
$$

where we defined

$$
\tan \theta_{0} \equiv \frac{g_{1}}{g_{2}}
$$

For $v_{\phi}^{2} \ll v_{\Delta}^{2}$, the values of $\tan \theta_{0}$ span essentially the same range as $\tan \theta$. The $W$ and $W^{\prime}$ masses, given in Eq. (3.23), have simple expressions to leading order in $v_{\phi} / v_{\Delta}$ :

$$
\begin{aligned}
& M_{W}=\frac{g_{2}}{2} v_{\phi} \sin \theta_{0}\left[1-\frac{v_{\phi}^{2}}{2 v_{\Delta}^{2}} \sin ^{4} \theta_{0}+O\left(v_{\phi}^{4} / v_{\Delta}^{4}\right)\right], \\
& M_{W^{\prime}}=\frac{g_{2} v_{\Delta}}{2 \cos \theta_{0}}\left[1+\frac{v_{\phi}^{2}}{2 v_{\Delta}^{2}} \sin ^{4} \theta_{0}+\frac{v_{\phi}^{4}}{8 v_{\Delta}^{4}}\left(4 \cot ^{2} \theta_{0}-1\right) \sin ^{8} \theta_{0}+O\left(v_{\phi}^{6} / v_{\Delta}^{6}\right)\right] .
\end{aligned}
$$

The low-energy charged current interactions are mediated in this model by both $W$ and $W^{\prime}$ exchange. Consequently, the Fermi constant is related to our parameters by

$$
\begin{aligned}
4 \sqrt{2} G_{F} & =\frac{\left(g_{1} \cos \theta\right)^{2}}{M_{W}^{2}}+\frac{\left(g_{1} \sin \theta\right)^{2}}{M_{W^{\prime}}^{2}} \\
& =\frac{g^{2}}{M_{W}^{2}}\left[1+\frac{v_{\phi}^{2}}{v_{\Delta}^{2}} \sin ^{4} \theta_{0}+O\left(v_{\phi}^{4} / v_{\Delta}^{4}\right)\right],
\end{aligned}
$$

where we used Eq. (3.24), which defines $g$ as the tree-level $W$ coupling to leptons and quarks. This shows that the measurements of the weak coupling in low-energy processes 
and in collider processes involving $W$ bosons should agree up to corrections of order $\left(v_{\phi} / v_{\Delta}\right)^{2} \sin ^{4} \theta_{0}$. Defining the weak scale $v \approx 246 \mathrm{GeV}$ through $G_{F}=2^{1 / 2} v^{-2}$, and using Eq. (3.29), we obtain the relation between the $\Phi$ VEV and the weak scale

$$
v=v_{\phi}\left[1-\frac{v_{\phi}^{2}}{v_{\Delta}^{2}} \sin ^{2} \theta_{0}+O\left(v_{\phi}^{4} / v_{\Delta}^{4}\right)\right] .
$$

\subsection{3 $\quad Z^{\prime}$ mass and couplings}

Electrically-neutral gauge bosons also acquire mass terms in the vacuum (3.2):

$$
\frac{v_{\phi}^{2}}{8}\left(g_{1} W_{1 \mu}^{3}-g_{Y} B_{\mu}\right)^{2}+\frac{v_{\Delta}^{2}}{8}\left(g_{2} W_{2 \mu}^{3}-g_{1} W_{1 \mu}^{3}\right)^{2} .
$$

It is convenient to diagonalize these in two steps. First, we define some intermediate fields denoted with hats:

$$
\begin{aligned}
& \hat{Z}_{\mu}^{\prime}=W_{2 \mu}^{3} \cos \theta_{0}-W_{1 \mu}^{3} \sin \theta_{0}, \\
& \hat{Z}_{\mu}=\left(W_{2 \mu}^{3} \sin \theta_{0}+W_{1 \mu}^{3} \cos \theta_{0}\right) \cos \hat{\theta}_{W}-B_{\mu} \sin \hat{\theta}_{W},
\end{aligned}
$$

where the angle $\hat{\theta}_{W}$ is defined in terms of coupling ratios:

$$
\tan \hat{\theta}_{W}=\frac{g_{Y}}{g_{2} \sin \theta_{0}} .
$$

The gauge boson orthogonal to $\hat{Z}_{\mu}$ and $\hat{Z}_{\mu}^{\prime}$ is the photon $\left(A_{\mu}=W_{1 \mu}^{3} \cos \theta_{0} \sin \hat{\theta}_{W}+\right.$ $\left.B_{\mu} \cos \hat{\theta}_{W}\right)$, already in the physical eigenstate. The measured electromagnetic coupling, $e=\sqrt{4 \pi \alpha} \approx 0.313$, is related to the original gauge couplings through

$$
g_{Y} \cos \hat{\theta}_{W}=e .
$$

The mass-squared matrix for $\hat{Z}_{\mu}$ and $\hat{Z}_{\mu}^{\prime}$ takes the form

$$
\mathcal{M}_{Z}^{2}=\frac{g_{2}^{2}}{4} \sin ^{2} \theta_{0}\left(\begin{array}{cc}
\frac{v_{\phi}^{2}}{\cos ^{2} \hat{\theta}_{W}} & -\frac{v_{\phi}^{2} \tan \theta_{0}}{\cos \hat{\theta}_{W}} \\
-\frac{v_{\phi}^{2} \tan \theta_{0}}{\cos \hat{\theta}_{W}} & \frac{4 v_{\Delta}^{2}}{\sin ^{2} 2 \theta_{0}}+v_{\phi}^{2} \tan ^{2} \theta_{0}
\end{array}\right) .
$$


In the second step, we rotate $\hat{Z}_{\mu}$ and $\hat{Z}_{\mu}^{\prime}$ by an angle $\epsilon_{Z}$, given by

$$
\tan 2 \epsilon_{Z}=\frac{v_{\phi}^{2} \sin 2 \theta_{0} \sin ^{2} \theta_{0} \cos \hat{\theta}_{W}}{v_{\Delta}^{2} \cos ^{2} \hat{\theta}_{W}+v_{\phi}^{2} \sin ^{4} \theta_{0}\left(\cos ^{2} \hat{\theta}_{W}-\cot ^{2} \theta_{0}\right)}
$$

in order to obtain the mass eigenstate $Z$ and $Z^{\prime}$ bosons:

$$
\begin{aligned}
& Z_{\mu}=\hat{Z}_{\mu} \cos \epsilon_{Z}+\hat{Z}_{\mu}^{\prime} \sin \epsilon_{Z}, \\
& Z_{\mu}^{\prime}=-\hat{Z}_{\mu} \sin \epsilon_{Z}+\hat{Z}_{\mu}^{\prime} \cos \epsilon_{Z} .
\end{aligned}
$$

The masses of the heavy neutral spin-1 particles are

$$
M_{Z, Z^{\prime}}=\frac{g_{2}}{2 \sqrt{2}}\left[\frac{v_{\Delta}^{2}}{\cos ^{2} \theta_{0}}+v_{\phi}^{2} \sin ^{2} \theta_{0}\left(\frac{1}{\cos ^{2} \hat{\theta}_{W}}+\tan ^{2} \theta_{0} \mp \frac{2 \tan \theta_{0}}{\sin 2 \epsilon_{Z} \cos \hat{\theta}_{W}}\right)\right]^{1 / 2}
$$

The tree-level results (3.33)- 3.40 have been obtained without approximations. Expanding now in $v_{\phi}^{2} / v_{\Delta}^{2}$, we find

$$
\begin{aligned}
& M_{Z}=\frac{g_{2} v_{\phi} \sin \theta_{0}}{2 \cos \hat{\theta}_{W}}\left[1-\frac{v_{\phi}^{2}}{2 v_{\Delta}^{2}} \sin ^{4} \theta_{0}+O\left(v_{\phi}^{4} / v_{\Delta}^{4}\right)\right], \\
& M_{Z^{\prime}}=\frac{g_{2} v_{\Delta}}{2 \cos \theta_{0}}\left[1+\frac{v_{\phi}^{2}}{2 v_{\Delta}^{2}} \sin ^{4} \theta_{0}+\frac{v_{\phi}^{4}}{8 v_{\Delta}^{4}}\left(4 \frac{\cot ^{2} \theta_{0}}{\cos ^{2} \hat{\theta}_{W}}-1\right) \sin ^{8} \theta_{0}+O\left(v_{\phi}^{6} / v_{\Delta}^{6}\right)\right] .
\end{aligned}
$$

The original five parameters from the gauge sector $\left(g_{1}, g_{2}, g_{Y}, v_{\phi}, v_{\Delta}\right)$ can be traded for three observables (e.g., e, $\left.s_{W}, M_{W}\right)$ and two parameters that can be measured once the $W^{\prime}$ or $Z^{\prime}$ boson is discovered $\left(M_{W^{\prime}}, \tan \theta\right)$, using Eqs. (3.27), (3.29), (3.30), (3.36) and

$$
s_{W}=\sin \hat{\theta}_{W}\left[1-\frac{v_{\phi}^{2}}{v_{\Delta}^{2}} \sin ^{2} \theta_{0} \cos ^{2} \theta_{0}+O\left(v_{\phi}^{4} / v_{\Delta}^{4}\right)\right] .
$$

Eqs. (3.41) and (3.42), combined with the above equation, show that the tree-level relation $M_{Z} c_{W}=M_{W}$, where $c_{W} \equiv \cos \theta_{W}$, is satisfied only up to corrections of order $v_{\phi}^{2} / v_{\Delta}^{2}$. Furthermore, the $Z$ couplings to fermions are modified at order $v_{\phi}^{2} / v_{\Delta}^{2}$ compared to the SM. Thus, the current agreement between electroweak measurements and the SM 
imposes an upper limit on $v_{\phi}^{2} / v_{\Delta}^{2}$, or equivalently, a lower limit on the $W^{\prime}$ mass for a fixed $\tan \theta$. The lower limit at the $95 \%$ CL given by the global fit performed in Ref. [75] increases from $M_{W^{\prime}} \gtrsim 600 \mathrm{GeV}$ for $\tan \theta=0.2$, to $M_{W^{\prime}} \gtrsim 2 \mathrm{TeV}$ for $\tan \theta=1$ (i.e., sequential $\left.W^{\prime}\right)$.

The relative mass splitting between $W^{\prime}$ and $Z^{\prime}$ is very small:

$$
\frac{M_{Z^{\prime}}}{M_{W^{\prime}}}-1=\frac{s_{W}^{2}}{2 c_{W}^{2}} \tan ^{2} \theta\left(\frac{M_{W}}{M_{W^{\prime}}}\right)^{4}+O\left(M_{W}^{6} / M_{W^{\prime}}^{6}\right)
$$

which is less than $6 \times 10^{-6}$ for $M_{W^{\prime}}>1 \mathrm{TeV}$ and $\tan \theta<1$. This implies that the $W^{\prime}$ mass and $\tan \theta$ will be constrained by both $Z^{\prime}$ and $W^{\prime}$ searches. The $Z^{\prime}$ interacts with the left-handed fermion doublets, with a coupling given by $g \tan \theta T^{3}$ plus corrections of order $v_{\phi}^{2} / v_{\Delta}^{2}$ that are different for quarks and leptons. The $Z^{\prime}$ couplings to $S U(2)_{W}$ singlets are suppressed by $v_{\phi}^{2} / v_{\Delta}^{2}$.

\section{3 $W^{\prime}$ and $Z^{\prime}$ decays}

The new gauge bosons interact with SM fermions and gauge bosons, as well as with the Higgs particles. Usually, resonance searches for new gauge bosons rely on sizable branching fractions of the $W^{\prime}$ and $Z^{\prime}$ decays into SM fermions. However, if the scalars are lighter than the vector bosons than the decays into SM fermions may be suppressed. In our model, the left-handed fermion doublets transform under $S U(2)_{1}$, while all fermions are singlets under $S U(2)_{2}$. Thus, the $W^{\prime}$ and $Z^{\prime}$ couplings to fermions are induced through mixing with the $W$ and $Z$, so that for small $\tan \theta$ decays to heavy scalars become important.

Neglecting corrections of $O\left(v_{\phi}^{2} / v_{\Delta}^{2}\right)$, the $W^{\prime}$ and $Z^{\prime}$ coupling to fermion doublets is given by $g \tan \theta$. The partial widths for decays to leptons (without summing over flavors)

$$
\Gamma\left(W^{\prime} \rightarrow \ell \nu\right) \approx 2 \Gamma\left(Z^{\prime} \rightarrow \ell^{+} \ell^{-}\right) \approx \frac{\alpha}{6 s_{W}^{2}} \tan ^{2} \theta M_{W^{\prime}}
$$


are suppressed for $0.2<\tan \theta<1$. By contrast, the $W^{\prime}$ and $Z^{\prime}$ couplings to pairs of odd Higgs particles are enhanced by $1 / \tan \theta$ :

$$
\begin{gathered}
g_{W^{\prime} H^{ \pm} A^{0}}=g_{Z^{\prime} H^{0} A^{0}}=\frac{g}{\sin 2 \theta}, \\
g_{W^{\prime} H^{ \pm} H^{0}}=g_{Z^{\prime} H^{+} H^{-}}=\frac{g}{\tan 2 \theta},
\end{gathered}
$$

where we ignored corrections of order $v_{\phi}^{2} / v_{\Delta}^{2}$. These couplings lead to the following partial widths:

$$
\begin{aligned}
\Gamma\left(W^{\prime} \rightarrow H^{ \pm} A^{0}\right) \approx \Gamma\left(Z^{\prime} \rightarrow H^{0} A^{0}\right) \approx \frac{\alpha M_{W^{\prime}}}{12 s_{W}^{2} \sin ^{2} 2 \theta}\left(1-2 \frac{M_{H^{+}}^{2}+M_{A^{0}}^{2}}{M_{W^{\prime}}^{2}}+\frac{\left(M_{H^{+}}^{2}-M_{A^{0}}^{2}\right)^{2}}{M_{W^{\prime}}^{4}}\right)^{3 / 2}, \\
\Gamma\left(W^{\prime} \rightarrow H^{ \pm} H^{0}\right) \approx \Gamma\left(Z^{\prime} \rightarrow H^{+} H^{-}\right) \approx \frac{\alpha M_{W^{\prime}}}{12 s_{W}^{2} \tan ^{2} 2 \theta}\left(1-4 \frac{M_{H^{+}}^{2}}{M_{W^{\prime}}^{2}}\right)^{3 / 2} .
\end{aligned}
$$

The $W^{\prime}$ can also decay into $W Z$ and $W h^{0}$ final states, but these partial widths are suppressed by $v_{\phi}^{4} / v_{\Delta}^{4}$.

Figure 3.1 shows the branching fractions of the $W^{\prime}$ and $Z^{\prime}$ as a function of $\tan \theta$ for the dominant channels. As a benchmark point, we have used $M_{W^{\prime}}=3 \mathrm{TeV}, M_{H^{+}}=300$ $\mathrm{GeV}$ and $M_{A}=200 \mathrm{GeV}$ (as shown in Section II, $M_{W^{\prime}}=M_{Z^{\prime}}$ and $M_{H^{+}}=M_{H^{0}}$ to a good accuracy). For $\tan \theta \lesssim 0.4$, the $W^{\prime}$ decays dominantly to pairs of odd Higgs particles. It is important to investigate collider signatures of these decays.

The heavier odd scalars decay into the LOP (taken to be $A^{0}$ ) and an electroweak boson, so that $W^{\prime}$ and $Z^{\prime}$ can each undergo two cascade decays: $W^{\prime} \rightarrow H^{+} A^{0} \rightarrow W A^{0} A^{0}$, $W^{\prime} \rightarrow H^{+} H^{0} \rightarrow W^{+} A^{0} Z A^{0}$ (see Figure 2), and $Z^{\prime} \rightarrow H^{0} A^{0} \rightarrow Z A^{0} A^{0}, Z^{\prime} \rightarrow H^{+} H^{-} \rightarrow$ $W^{+} A^{0} W^{-} A^{0}$.

If the $\mathbb{Z}_{2}$ symmetry discussed in Section 3.2 is exact, then $A^{0}$ is a component of dark matter. We will not explore here the constraints on the parameter space from the upper limit on relic density, nor from direct detection experiments (nuclear scattering would occur through Higgs exchange and gauge boson loops); these constraints can be in any case relaxed by allowing a tiny $\mathbb{Z}_{2}$ violation in the scalar potential. While an in-depth exploration of this model as an explanation for dark matter is left for future 

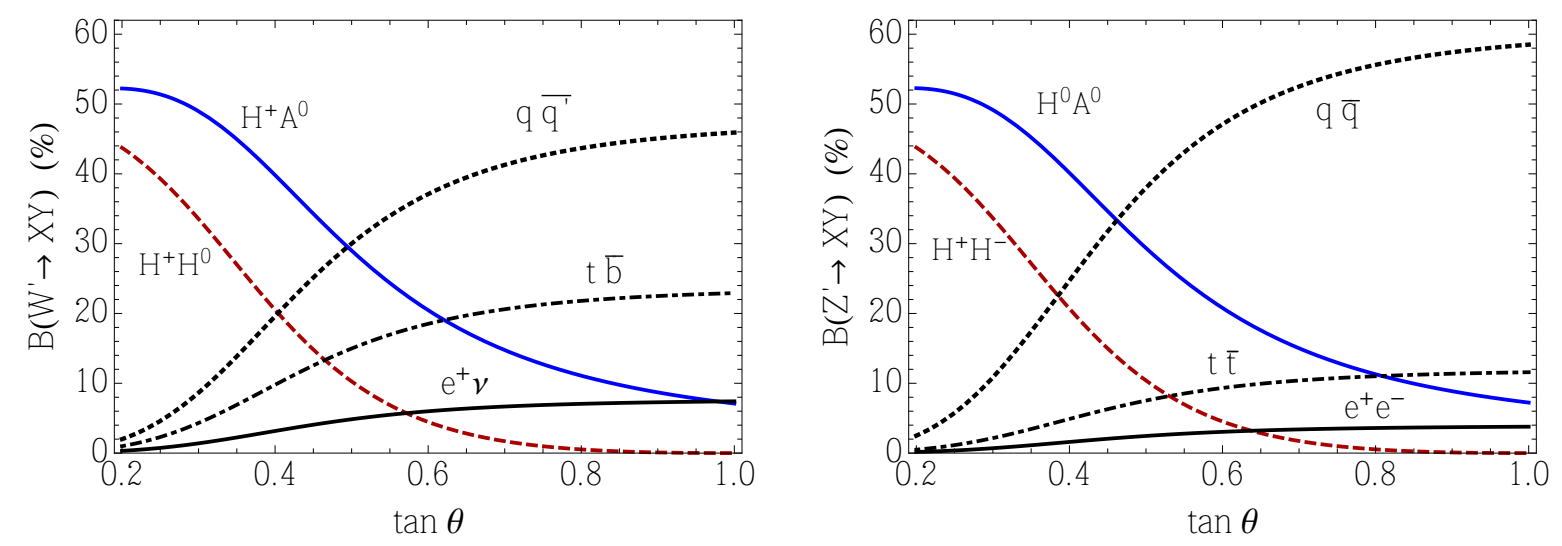

Figure $3.1 W^{\prime}$ and $Z^{\prime}$ branching fractions as a function of mixing angle, for $M_{W^{\prime}}=3$ $\mathrm{TeV}, M_{H^{+}}=300 \mathrm{GeV}, M_{A}=200 \mathrm{GeV}$.

work, we note that it shares many features with inert doublet [78] and minimal dark matter scenarios [77].

The possibility that the $\mathbb{Z}_{2}$ symmetry is violated by terms in the scalar potential of the type

$$
\operatorname{Tr}\left(\Delta^{\dagger} \tilde{\Delta}\right), \Phi^{\dagger} \tilde{\Delta}^{\dagger} \tilde{\Delta} \Phi, \quad \operatorname{Tr}\left(\Delta^{\dagger} \Delta \Delta^{\dagger} \tilde{\Delta}\right), \operatorname{Tr}\left(\Delta^{\dagger} \Delta\right) \operatorname{Tr}\left(\Delta^{\dagger} \tilde{\Delta}\right)
$$

is also worth considering. The weak-triplet scalar $\left(H^{ \pm}, H^{0}\right)$ as well as the singlet $A^{0}$ would mix with the $\Phi$ doublet, allowing direct two-body decays of $A^{0}, H^{0}$ and $H^{ \pm}$to SM particles. Furthermore, the three CP-even neutral scalars $\left(H^{0}, h, H^{\prime}\right)$ would then mix, so that $W^{\prime}$ and $Z^{\prime}$ decays involving the SM-like Higgs boson are possible. These include $W^{\prime} \rightarrow H^{+} h^{0}$ with $H^{+} \rightarrow t \bar{b}$ (this channel is analyzed in [79]), as well as $W^{\prime} \rightarrow$ $H^{+} h^{0} \rightarrow W^{+} A^{0} h^{0}$ and $Z^{\prime} \rightarrow h^{0} A^{0}$ with $A^{0} \rightarrow b \bar{b}$ (or $h^{0} Z$ or $t \bar{t}$ if kinematically allowed). There are, however, various constraints on deviations from the SM Higgs couplings, implying that the $\mathbb{Z}_{2}$ violating mixing is small, so that we expect that the above final states have relatively small branching fractions.

It is also interesting to consider the intermediate case, where the violation of $\mathbb{Z}_{2}$ is very small, i.e., the coefficients of the operators (3.4) are much less than one. In that case all $W^{\prime}$ and $Z^{\prime}$ cascade decays through the odd Higgs particles proceed as before, 

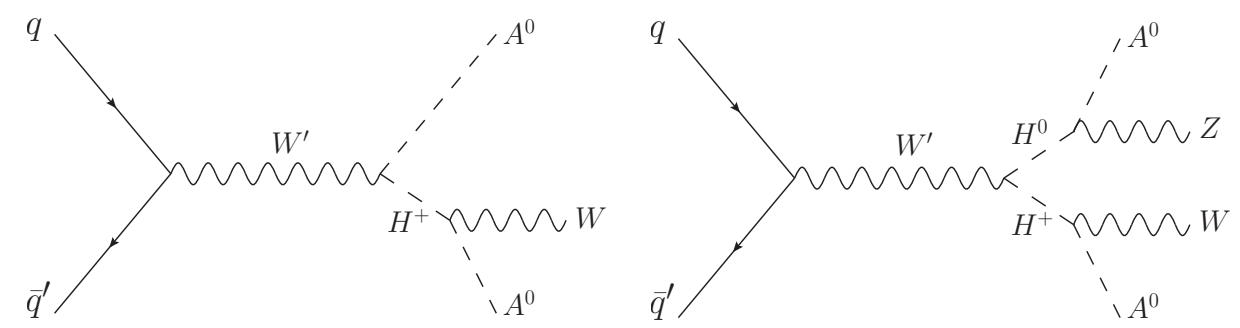

Figure $3.2 W^{\prime}$ production and cascade decays through odd Higgs particles.

but the $A^{0}$ would decay to a pair of heaviest fermions of mass below $M_{A} / 2$. This leads to a variety of noteworthy final states: $W^{\prime} \rightarrow W Z+4 b, Z^{\prime} \rightarrow Z+4 b$, or $W^{\prime} \rightarrow W Z t \bar{t} t \bar{t}$, $Z^{\prime} \rightarrow Z t \bar{t} t \bar{t}$, etc. For a range of parameters, the decays of $A_{0}$ may be displaced but still within the detector, leading to potentially confusing events. In what follows we will consider only the case where $A^{0}$ is stable enough to escape the detector.

\subsection{LHC signatures with stable $A^{0}$}

At the LHC, the $W^{\prime}$ boson would be mainly produced in the $s$ channel from quarkantiquark initial state, even for small $\tan \theta$. In the narrow width approximation, the leading-order cross section for $W^{\prime}$ production followed by decay into $H^{+} A^{0}$ or $H^{+} H^{0}$ is

$$
\sigma\left(p p \rightarrow W^{\prime} \rightarrow H^{+} A^{0}, H^{+} H^{0}\right) \approx \frac{\alpha \tan ^{2} \theta}{24 s_{W}^{2} s} w\left(M_{W^{\prime}}^{2} / s, \mu\right) B\left(W^{\prime} \rightarrow H^{+} A^{0}, H^{+} H^{0}\right)
$$

where

$$
w(z, \mu)=\sum_{i, j=1}^{2}\left|V_{i j}^{\mathrm{CKM}}\right|^{2} \int_{x}^{1} \frac{d x}{x}\left[u_{i}(x, \mu) \bar{d}_{j}\left(\frac{z}{x}, \mu\right)+\bar{u}_{i}(x, \mu) d_{j}\left(\frac{z}{x}, \mu\right)\right] .
$$

The functions $u_{i}(x, \mu)$ and $d_{i}(x, \mu)$ are the proton parton distribution functions for upand down-type quarks of the $i$ th generation at factorization scale $\mu$. Although QCD corrections to $W^{\prime}$ production are usually significant [80], in our case they are somewhat reduced due to the smaller $\alpha_{s}$ at the large values of $M_{W^{\prime}}$ that are relevant here. The effect of higher-order contributions can be approximated by the inclusion of a $K$-factor of approximately 1.3, with some scale dependence [20, 81]. 


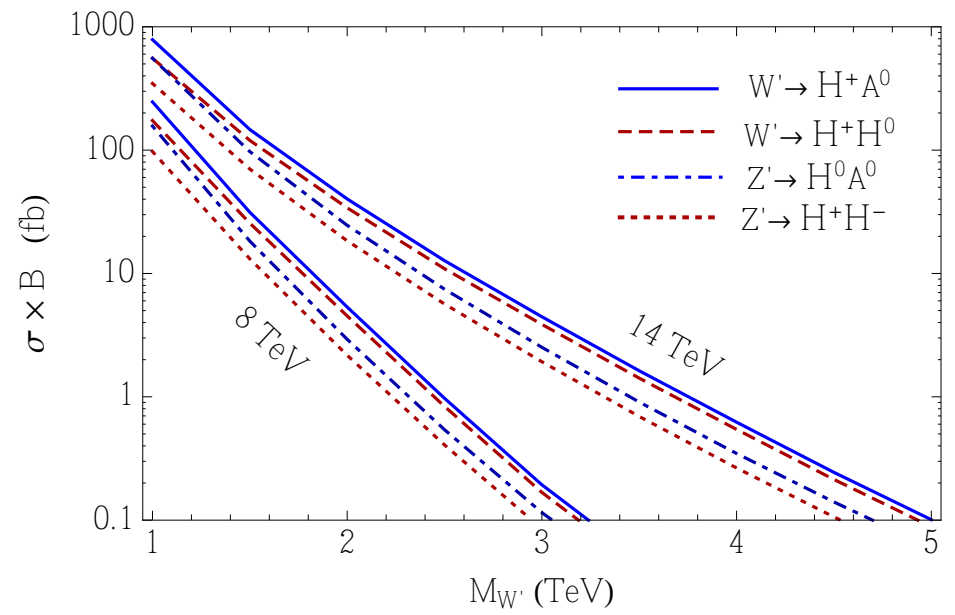

Figure 3.3 Leading-order cross sections times branching fractions for the processes $p p \rightarrow W^{\prime} \rightarrow H^{+} A^{0}, H^{+} H^{0}$ and $p p \rightarrow Z^{\prime} \rightarrow H^{0} A^{0}, H^{+} H^{-}$at $\sqrt{s}=8 \mathrm{TeV}$ and $14 \mathrm{TeV}$. We have chosen $\tan \theta=1 / 4, M_{H^{+}}=300 \mathrm{GeV}$ and $M_{A}=200 \mathrm{GeV}$.

Figure 3.3 shows the total cross section for the $p p \rightarrow W^{\prime} \rightarrow H^{+} A^{0}$ and $p p \rightarrow$ $W^{\prime} \rightarrow H^{+} H^{0}$ processes at $\sqrt{s}=8$ and $14 \mathrm{TeV}$, with $\tan \theta=0.25$. To compute these cross sections, we used FeynRules [83] for generating vertices from our Lagrangian, and input these into MadGraph 5 [82] (with parton distribution functions CTEQ6L1 [84]), which includes interference between the $W^{\prime}$ and $W$ contributions. We used the default MadGraph 5 prescription for dynamically setting the factorization and renormalization scales (typically $\mu \approx M_{W^{\prime}}$ ). We have set $M_{H^{+}}=300 \mathrm{GeV}, M_{A}=200 \mathrm{GeV}$; the cross sections are only weakly sensitive to the scalar masses as long as $W^{\prime}$ is much heavier. Figure 3.3 also shows the cross sections for $p p \rightarrow Z^{\prime} \rightarrow H^{0} A^{0}$ and $p p \rightarrow Z^{\prime} \rightarrow H^{+} H^{-}$, for the same parameters.

We assume that the $\mathbb{Z}_{2}$ symmetry discussed in Section 3.2 is sufficiently preserved so that the LOP escapes the detector. As noted there, $A^{0}$ is most likely the LOP, so that each of the above processes includes two $A^{0}$ in the final state, which appear as missing transverse energy $\left(E_{T}\right)$ in the detector. If $M_{W^{\prime}} \gg M_{H^{+}}$, then the $W$ or $Z$ boson emmited in the cascade decays $W^{\prime} \rightarrow H^{+} A^{0} \rightarrow W^{+} A^{0} A^{0}$ and $Z^{\prime} \rightarrow H^{0} A^{0} \rightarrow Z A^{0} A^{0}$ is highly boosted, carrying energy roughly equal of $M_{W^{\prime}} / 4$. This implies that hadronic 


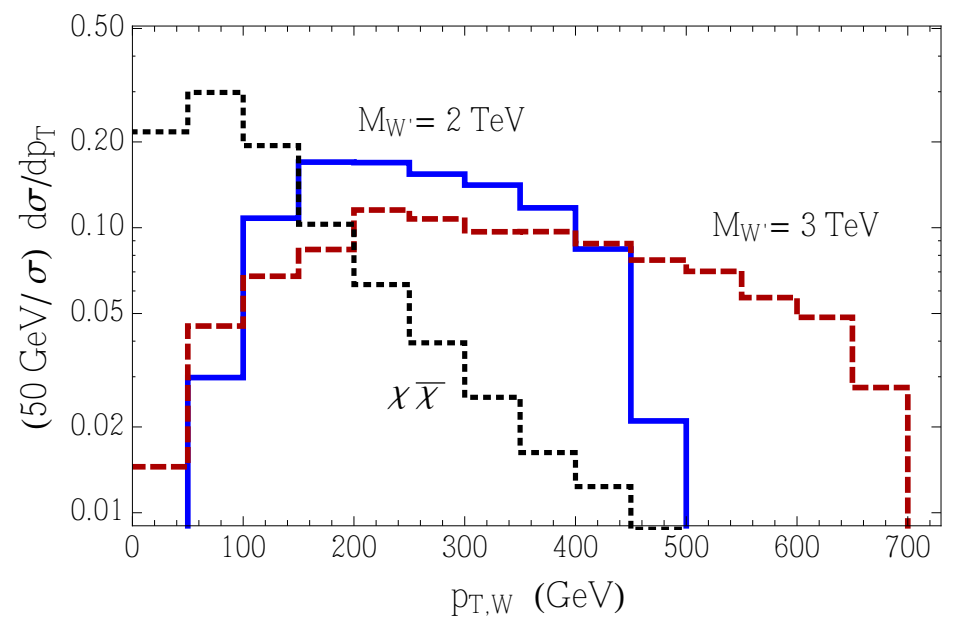

Figure 3.4 Transverse momentum distribution of the $W$ produced in $p p \rightarrow W^{\prime} \rightarrow H^{+} A^{0} \rightarrow W A^{0} A^{0}$ when $M_{W^{\prime}}=2 \mathrm{TeV}$ (solid blue line) and $M_{W^{\prime}}=3$ $\mathrm{TeV}$ (dashed red line), for $\sqrt{s}=8 \mathrm{TeV}, M_{H^{+}}=300 \mathrm{GeV}$ and $M_{A}=200 \mathrm{GeV}$. For comparison, the $W p_{T}$ distribution (dotted black line) is included for $p p \rightarrow W \chi \bar{\chi}$ through a $\bar{q} \gamma_{\mu} q \bar{\chi} \gamma^{\mu} \chi$ contact interaction (for $m_{\chi}=100 \mathrm{GeV}$ ).

decays of the $W$ or $Z$ boson lead to an interesting signature with the two jets collimated into a single wide jet with substructure, plus $\mathbb{E}_{T}$.

The ATLAS collaboration [85] has searched for this type of signature in the case of DM particles pair produced through a contact interaction to quarks [86, 87]. Compared to our model, the processes $p p \rightarrow W \bar{\chi} \chi$ and $p p \rightarrow Z \bar{\chi} \chi$ give rise to a smaller transverse momentum for the electroweak boson, which is radiated from an initial state quark. In Figure 3.4 we show the $p_{T}$ distributions for the $W$ arising from $W^{\prime} \rightarrow H^{+} A^{0} \rightarrow W^{+} A^{0} A^{0}$, as well as from initial state radiation in the case of a $\bar{q} \gamma_{\mu} q \bar{\chi} \gamma^{\mu} \chi$ contact interaction (for a Dirac fermion $\chi$ of mass $m_{\chi}=100 \mathrm{GeV}$ ). It is clear that the efficiency for a stringent $p_{T}(W)$ cut is much higher for our $W^{\prime}$ decays than in the case of contact interactions.

The cascade decays $W^{\prime} \rightarrow H^{+} H^{0} \rightarrow W^{+} A^{0} Z A^{0}$ and $Z^{\prime} \rightarrow H^{+} H^{-} \rightarrow W^{+} A^{0} W^{-} A^{0}$ lead to two highly boosted electroweak bosons plus $\mathbb{E}_{T}$. Hadronic decays of these $W$ and $Z$ bosons allow the use of substructure techniques [88] to reduce the QCD background. Both cascade decays would then give the same signal: two fat jets (each due to the two collimated jets produced by one of the bosons) plus missing transverse energy. 
The boosted $W$ and $Z$ "jets" plus $\mathbb{E}_{T}$ channel have the largest branching fractions. Nonetheless, leptonic decays of the boosted $W$ and $Z$ are also promising due to small backgrounds. These lead to final states with one, two or three leptons, plus $\mathbb{E}_{T}$.

The mono-lepton signature has been studied theoretically [89] and searched for at the LHC [90] in the case of contact interactions. Again, in our case the $W$ producing the lepton is generically more boosted. Unlike $W^{\prime}$ decays directly to a lepton-neutrino pair, there will be no Jacobian peak in the missing transverse energy distribution, as the $A^{0}$ 's carry away a substantial fraction of the energy of the $W^{\prime}$. In fact, the distribution will be peaked at low- $p_{T}$. Furthermore, if the masses of the $A^{0}$ and $H^{+}$are similar, the transverse momenta of the two final-state $A^{0}$ particles will have similar magnitudes but opposite directions, so their contribution to the $\mathbb{E}_{T}$ of the event is reduced. In this case, the missing energy distribution could look like a SM $W$ decay. This problem is mitigated if the $A^{0}$ is substantially lighter than the Higgs triplet states, in which case the $\mathbb{E}_{T}$ distribution will have a longer tail.

We simulate $W^{\prime}$ signals using Madgraph 5 [82], including showering and hadronization with Pythia 6.4 [91], and PGS detector simulation [92]; then we analyze the events with the MadAnalysis package [93]. Figure 3.5 (left panel) shows missing transverse energy distributions for $M_{H^{0}}=300 \mathrm{GeV}$ and $M_{H^{0}}=1 \mathrm{TeV}$, leaving the other parameters fixed at $M_{W^{\prime}}=3 \mathrm{TeV}, M_{A^{0}}=200 \mathrm{GeV}, \tan \theta=1 / 4$. The transverse mass distribution, which is used in LHC $W^{\prime}$ searches, is also peaked at small $M_{T}$. Moreover, the distribution does not change substantially for different values of the Higgs masses, as shown in Figure 3.5 (right panel). Therefore, the transverse mass is not the best observable for a $W^{\prime}$ decaying through odd Higgs particles.

A better observable for the single-lepton process $W^{\prime} \rightarrow H^{+} A^{0} \rightarrow W^{+}+E_{T}$ is the separation in azimuthal angle between the missing transverse energy and the lepton transverse momentum, $\Delta \phi_{p_{T}^{\ell}, E_{T}}$. When a $W$ or $W^{\prime}$ decays directly to a lepton-neutrino pair, the decay products are nearly back-to-back; for both the $W^{\prime}$ and dark matter mono-lepton analyses, CMS requires that $\Delta \phi_{p_{T}^{\ell}, \mathscr{F}_{T}}>0.8 \pi[20$. However, the kinematics 

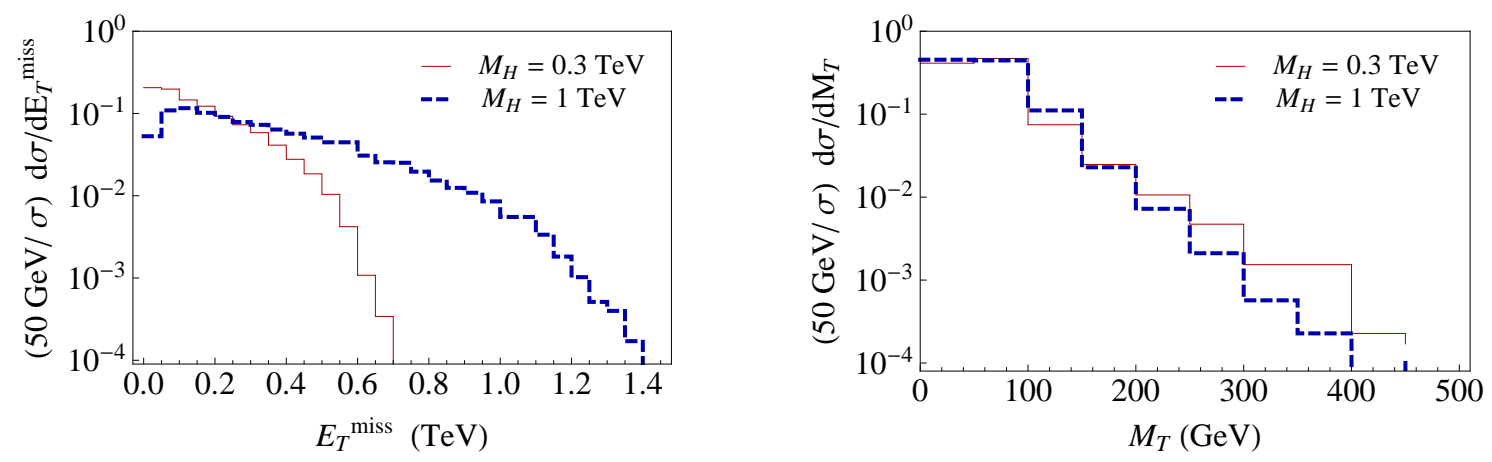

Figure 3.5 $\mathbb{E}_{T}$ distribution (left) and transverse mass (right) distributions for $M_{H^{0}}=300 \mathrm{GeV}$ and $M_{H^{0}}=1 \mathrm{TeV}$, all other parameters fixed $\left(M_{W^{\prime}}=3 \mathrm{TeV}\right.$, $\left.M_{A^{0}}=200 \mathrm{GeV}, \tan \theta=1 / 4\right)$.

for the decay $W^{\prime} \rightarrow A^{0} A^{0} l \nu$ are substantially different, with the $\Delta \phi_{p_{T}^{\ell}, E_{T}}$ distribution peaked at moderate-to-small values of $\Delta \phi_{p_{T}^{\ell}, \mathscr{E}_{T}}$; see Figure 3.6. In the rest frame of the $W^{\prime}, \vec{p}_{T}^{l}=-\sum \vec{p}_{T}^{m i s s}$, but in the lab frame, the $W^{\prime}$ transverse momentum is distributed among the four decay products and the correlation in azimuthal angle is lost.

There are also two processes leading to $\ell^{+} \ell^{-}+\mathbb{E}_{T}$. One of them is the $Z^{\prime} \rightarrow H^{0} A^{0} \rightarrow$ $Z A^{0} A^{0}$ cascade decay, with $Z \rightarrow \ell^{+} \ell^{-}$; the related process in the case of contact interactions has been discussed in [94]. The other one is $Z^{\prime} \rightarrow H^{+} H^{-} \rightarrow W^{+} A^{0} W^{-} A^{0}$ with leptonic $W$ decays; a similar final state, but without $s$-channel resonance, arises from chargino pair production Ref. [95].

The limits on our model set by current LHC results are already stronger than those from electroweak fits mentioned in Section 3.2. The searches in the $W^{\prime} \rightarrow \ell \nu$ channel, although affected by suppressed branching fraction for small $\tan \theta$, set relevant bounds. In Figure 3.7, we reinterpret the 95\% CL limit set by the CMS Collaboration [20] on $\sigma_{\text {excl. }} / \sigma_{\mathrm{SSM} W^{\prime}}$ as a limit on $\tan \theta$.

Existing LHC searches for other processes set less stringent limits. For $W^{\prime} \rightarrow$ $H^{+} A^{0} \rightarrow W^{+} A^{0} A^{0} \rightarrow \ell+\mathbb{E}_{T}$, we use Figure 4 of [90] to estimate the number of background events with $1<\Delta \phi_{p_{T}^{\ell}, \mathbb{E}_{T}}<1.5$ in the muon channel, then set an upper limit 


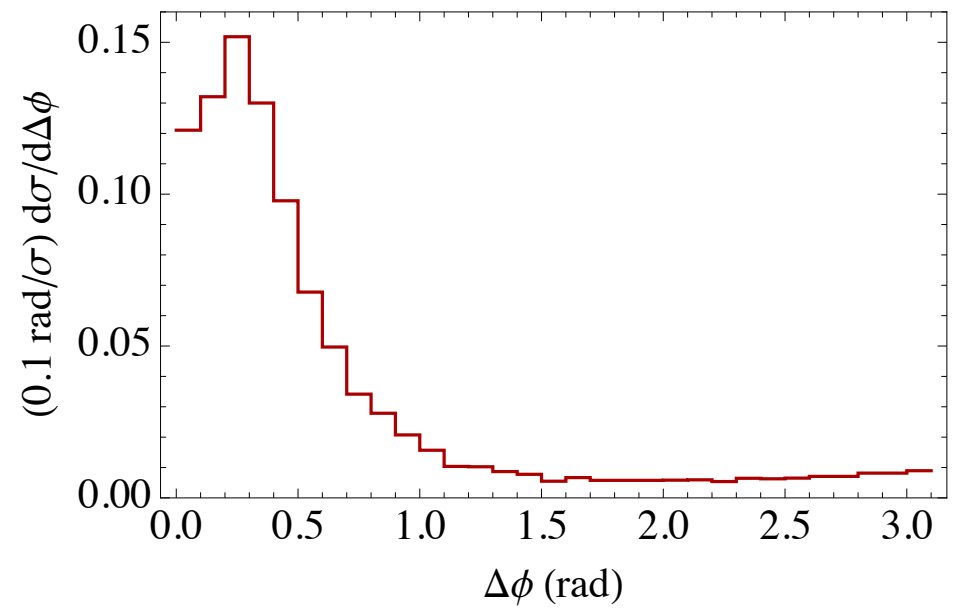

Figure 3.6 $\Delta \phi_{p_{T}^{\ell}, E_{T}}$ distribution, for $M_{H^{+}}=300 \mathrm{GeV}, M_{A}=200 \mathrm{GeV}$, and $\tan \theta=0.25$.

on $W^{\prime} \rightarrow \mu+\mathbb{E}_{T}$ events in the same region assuming no excess is observed. This limit is at most $M_{W^{\prime}}>1.05 \mathrm{TeV}$ for any $\tan \theta \leq 1$.

For the process $W^{\prime} \rightarrow H^{+} H^{0} \rightarrow W^{+} A^{0} Z A^{0} \rightarrow \ell^{+} \ell^{+} \ell^{-}+\mathbb{E}_{T}$, we use results from leptonic searches for charginos and neutralinos in [96]. We consider the search for a sameflavor, opposite sign electron or muon pair on the $Z$ peak $\left(75 \mathrm{GeV}<M_{\ell \ell}<105 \mathrm{GeV}\right.$ ), plus an additional electron or muon. We sum over $M_{T}$ bins, then we use a Poisson likelihood function multiplied over $\mathbb{E}_{T}$ bins to set an upper bound on the number of events. The upper limits on the number of $W^{\prime}$ events in each channel are then translated to a limit on $\tan \theta$ as a function of $M_{W^{\prime}}$. This limit is rather weak: for $M_{W^{\prime}}=1 \mathrm{TeV}$, only values of $\tan \theta>3.5$ are excluded.

We see that the search for direct decays to lepton plus $\mathbb{E}_{T}$ final states still provides the most stringent constraint on our model. The reason is that the 3-lepton rate is suppressed by both the $W \rightarrow \ell \nu$ and $Z \rightarrow \ell^{+} \ell^{-}$branching fractions. Furthermore, for the mono-lepton search, the selection cuts that optimize signal over background for $W \rightarrow \ell \nu$ cut out a substantial portion of the $H^{ \pm} A^{0}$ events. A new analysis focusing on the small $\Delta \phi_{p_{T}^{\ell}, \mathbb{E}_{T}}$ region, using both the electron and muon channels, would provide a stronger limit. 


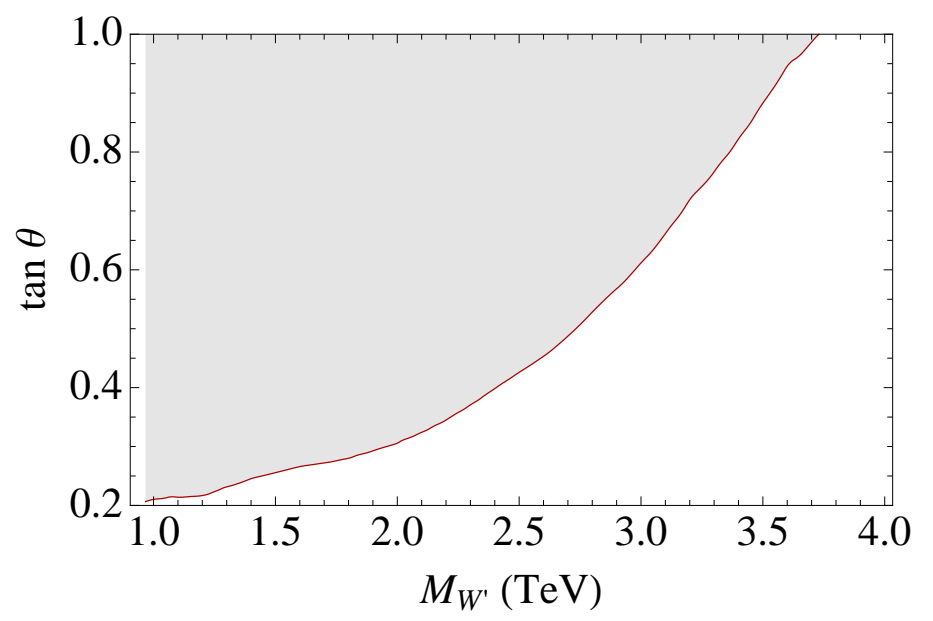

Figure 3.7 Exclusion limit in the $M_{W^{\prime}}-\tan \theta$ plane, derived from the CMS $W^{\prime} \rightarrow \ell \nu$ search [20], for $M_{H}=300 \mathrm{GeV}$ and $M_{A}=200 \mathrm{GeV}$.

Given the mass degeneracy between $W^{\prime}$ and $Z^{\prime}$, limits set by searches for $Z^{\prime} \rightarrow \ell^{+} \ell^{-}$ can also be plotted in the $M_{W^{\prime}}-\tan \theta$ plane. However, they are weaker than those from $W^{\prime} \rightarrow \ell \nu$ because both the production cross section and the leptonic branching fraction are smaller for $Z^{\prime}$ than for $W^{\prime}$.

\subsection{Summary of $W^{\prime}$ Analysis}

The $S U(2)_{1} \times S U(2)_{2} \times U(1)_{Y}$ model with a bidoublet and a doublet complex scalars is a simple renormalizable model that can serve as a benchmark for various LHC searches. It includes a meta-sequential $W^{\prime}$ boson whose $s$-channel production interferes constructively with the $W$ contribution, and depends on only two parameters: $M_{W^{\prime}}$ and the overall coupling normalization, $\tan \theta$. It also includes a $Z^{\prime}$ boson (degenerate in mass with $W^{\prime}$ ) which couples, to a good approximation, only to left-handed fermions.

The potential for the bidoublet $(\Delta)$ and doublet $(\Phi)$ scalars is chosen to be invariant under a $\mathbb{Z}_{2}$ transformation that interchanges the bidoublet and its charge conjugate. The physical scalar spectrum then consists of four odd Higgs particles (a mass-degenerate weak-triplet $H^{+}, H^{0}, H^{-}$, and a CP-odd singlet $A^{0}$ ), the recently discovered Higgs boson 
$\left(h^{0}\right)$, and a heavier scalar $\left(H^{\prime}\right)$ whose couplings to SM fields are the same as those of $h^{0}$ except for an universal suppression. The $A^{0}$ is naturally the LOP because a global $U(1)$ symmetry becomes exact in the $M_{A} \rightarrow 0$ limit.

The phenomenology of the scalars is worth exploring whether or not the $W^{\prime}$ and $Z^{\prime}$ bosons are light enough to be produced at the LHC. Electroweak production of the triplet scalars, for example, would lead to final states involving one or two weak bosons and two LOPs.

The range of parameters where $A^{0}$ is a viable dark matter particle remains to be studied. For the present work we focused on the case where $A^{0}$ is sufficiently long-lived to escape the detector, but we also mentioned possible signatures in the case where $A^{0}$ decays (promptly or with a displaced vertex) into fermion pairs.

This model illustrates nicely the possibility that the $W^{\prime}$ and $Z^{\prime}$ bosons may decay predominantly (with branching fraction as large as 96\%) into the scalars responsible for breaking the extended gauge symmetry. Generically, the high-energy behavior of any $W^{\prime}$ boson requires it to be associated with a non-Abelian gauge symmetry (or else it must be a bound state with the compositeness scale not much higher than its mass), which in turn implies a larger Higgs sector. The non-Abelian gauge coupling can be significantly larger than the Higgs quartic couplings, implying vector bosons much heavier than the scalars.

In our model, the $W^{\prime}$ and $Z^{\prime}$ couplings to the odd Higgs particles are enhanced for $\tan \theta \ll 1$ by $1 / \tan \theta$. Consequently, the usual $u \bar{d} \rightarrow W^{\prime} \rightarrow \ell \nu$ or $t \bar{b}$ channels currently used in searches at the LHC are suppressed both in production and in braching fractions, the combined effect being of order $\tan ^{6} \theta$. The mass limits on a sequential $W^{\prime}$, currently around $3.8 \mathrm{TeV}$, are relaxed for $\tan \theta \approx 0.2$ (the lower perturbativity bound) to $M_{W^{\prime}}>1 \mathrm{TeV}$. At the same time, the cascade decays through odd Higgs particles, $W^{\prime} \rightarrow H^{+} A^{0} \rightarrow W^{+} A^{0} A^{0}, \quad W^{\prime} \rightarrow H^{+} H^{0} \rightarrow W^{+} A^{0} Z A^{0}, \quad Z^{\prime} \rightarrow H^{0} A^{0} \rightarrow Z A^{0} A^{0}$ and $Z^{\prime} \rightarrow H^{+} H^{-} \rightarrow W^{+} A^{0} W^{-} A^{0}$ allow interesting searches at the LHC, with boosted $W$ and $Z$ bosons decaying either hadronically or leptonically. 


\section{Chapter 4}

\section{Measuring the 2HDM scalar potential}

\subsection{Introduction}

Particle physics is at a crossroads. The discovery of the $125.5 \mathrm{GeV}$ Higgs boson at the LHC [97, 98] validates the fundamental theoretical tenet that the electroweak gauge symmetry is spontaneously broken. The LHC measurements of the Higgs couplings to weak bosons, photons, gluons and fermions are all consistent with their Standard Model (SM) predicted values [99, 100, 101]. This is satisfying, to a degree, but it is also mystifying that the SM should work so well. The Higgs mass is not predicted in the SM, and the large hierarchy of the electroweak and Planck scales is unexplained by the SM. The commonly expected explanation for the hierarchy - physics beyond the SM at the $\mathrm{TeV}$ scale - has not been borne out thus far by the LHC experiments at 7 and $8 \mathrm{TeV} \mathrm{cm}$ energy. The LHC upgrade to $14 \mathrm{TeV}$ (LHC14), with 10 times the present luminosity, may change this situation by the discovery of new particles. Regardless, the properties of the Higgs boson will be central in the search for new physics (for a recent overview, see [102]). The Higgs potential itself has so far not been subject to experimental scrutiny, since this requires the more challenging measurements of triple and quartic Higgs selfinteractions via pair production of Higgs bosons. However, this important avenue of pursuit should soon be possible with data from the upcoming LHC14 run. 
The Two Higgs Doublet Model (2HDM) (see e.g. [103, 18, 104]) provides a convenient general framework in which to explore extensions of the SM and to characterize deviations of the Higgs couplings from their SM values in analyses of experimental data. For the case of a CP-conserving Higgs potential, the three physical neutral Higgs states consist of $2 \mathrm{CP}$-even states, $h$ and $H$, and a CP-odd state $A$. The pairproduction of these Higgs bosons is the means by which the Higgs potential can be experimentally determined and signs of new physics may be found [105, 106, 107, 108]. The goal of our study to is assess in which final states the Higgs pair production processes, especially $h h$ and $h H$, can be measured within a specific class of 2HDMs, the Type-II 2HDM (see e.g. [109, 110, 111, 112, 113]), for which one of the Higgs doublets has tree-level couplings only to up-type quarks and the other has tree-level couplings only to down-type quarks and leptons (and thus it includes the minimal supersymmetric standard model as a special case). Beyond this, a determination of the Higgs self-couplings can be made to some degree [108, 114, 115]. Implicit in this strategy is that the generalization of the Higgs sector is the only modification of new physics signals of relevance to Higgs pair production and decay; we will not consider scenarios where new physics in other sectors affects the production of Higgs pairs, as found in [116, 117, 118, 119, 120, 121, 122, 123, 124, 125, 126, 127, 128, 129, 130, 131].

The layout of our study is as follows. In Section 4.2, we briefly describe the 2HDM, discuss the present constraints relevant for our study, and introduce three benchmark points to help elucidate discovery prospects. In Section 4.4, we review and present the analytic formulae for the pair production of the Higgs bosons in gluon-gluon fusion, which is the dominant sub-process in $p p$ collisions at the LHC. There are two classes of contributing Feynman diagrams: s-channel Higgs boson exchange and a box diagram with a top-quark loop, as shown in Fig. 4.1. Representative Higgs pair production crosssections are provided in this section. In Section 4.5, we describe our simulation of the $h h$ subprocess and subsequent $h$ decays. We then proceed with a systematic consideration of the possible decay channels of the $h h$ along with their backgrounds from the relevant 
SM processes. We then describe the Multi-Variate Analysis (MVA) methodology that is the basis of our extraction of the signal from the background. The MVA methodology distinguishes signal from background by kinematics, and takes multiple variables into account simultaneously.

In Section 4.6, we turn to a study of associated $h H$ production, which we find to be complementary to the resonant $H \rightarrow h h$ production. In the $h H$ process, both the triangle and box diagrams in Fig. 4.1 contribute, with contributions of both $h^{*}$ and $H^{*}$ in the s-channel. The triangle diagrams provide sensitivity to the products of the top-Yukawa and the $\lambda^{h h H}$ tri-scalar couplings. Depending on its mass, the heavy scalar, $H$, has several available decay channels that can potential provide identifiable signals, including $b \bar{b}, W W^{*}, Z Z^{*}$, and $t \bar{t}$. The decay branching fractions of $H$ to these channels are dependent on the mass and Higgs mixing parameters. We perform simulations of these channels and their SM backgrounds to assess the discovery prospects. We find that the following channels all lead to a possible discovery: $h H \rightarrow b \bar{b} \gamma \gamma, b \bar{b} b \bar{b}, Z Z b \bar{b}, b \bar{b} t \bar{t}$ and $b \bar{b} b \bar{b} \gamma \gamma$, allowing for a rich variety of measurements. Finally, in Section 4.7, we summarize our results. In our evaluation of the reach of LHC14, we assume throughout an integrated luminosity of $3 \mathrm{ab}^{-1}$.

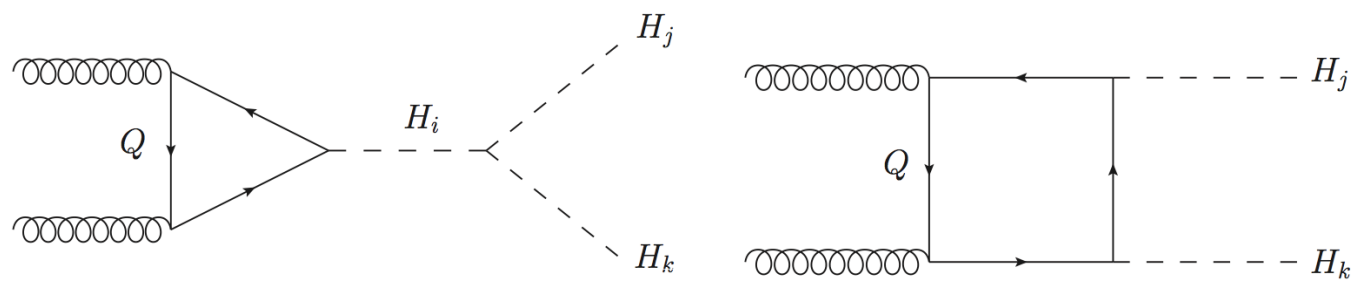

Figure 4.1 Representative Feynman diagrams which contribute to Higgs boson pair production.

\subsection{The Two Higgs Doublet Model}

In this section, we will provide a very brief overview of the $2 \mathrm{HDM}$ and the theoretical constraints on the potential (for more comprehensive discussions, see e.g. [103, 18, 104]). 
The model consists of two Higgs doublets, which we express as the opposite-hypercharge Higgs doublets $\Phi_{1,2}$ as follows:

$$
\Phi_{1}=\left(\begin{array}{c}
\left(\phi_{1}^{0}+v_{1}-i \eta_{1}^{0}\right) / \sqrt{2} \\
-\phi_{1}^{-}
\end{array}\right), \quad \Phi_{2}=\left(\begin{array}{c}
\phi_{2}^{+} \\
\left(\phi_{2}^{0}+v_{2}+i \eta_{2}^{0}\right) / \sqrt{2}
\end{array}\right)
$$

in which the vacuum expectation values (vevs) $v_{1,2}$ satisfy the relation $v=\sqrt{v_{1}^{2}+v_{2}^{2}}=$ $246 \mathrm{GeV}$. We follow standard practice and assume for simplicity both that CP is conserved (i.e., is not explicitly or spontaneously broken), and that the theory obeys a softly broken $Z_{2}$ symmetry that eliminates quartic terms that are odd in either of the doublets, but allows a quadratic term that mixes $\Phi_{1}$ and $\Phi_{2}$ (this is consistent with our eventual specialization to the Type II 2HDM; see e.g. [18] for a detailed discussion of these issues). With these assumptions, the scalar potential takes the following form:

$$
\begin{aligned}
V & =m_{1}^{2} \Phi_{1}^{\dagger} \Phi_{1}+m_{2}^{2} \Phi_{2}^{\dagger} \Phi_{2}-\frac{1}{2} M^{2} \sin 2 \beta\left(\Phi_{1}^{\dagger} \tilde{\Phi}_{2}+\tilde{\Phi}_{2}^{\dagger} \Phi_{1}\right)+\frac{\lambda_{1}}{2}\left|\Phi_{1}^{\dagger} \Phi_{1}\right|^{2}+\frac{\lambda_{2}}{2}\left|\Phi_{2}^{\dagger} \Phi_{2}\right|^{2} \\
& +\lambda_{3}\left|\Phi_{1}^{\dagger} \Phi_{1} \Phi_{2}^{\dagger} \Phi_{2}\right|+\lambda_{4}\left|\Phi_{1}^{\dagger} \tilde{\Phi}_{2} \tilde{\Phi}_{2}^{\dagger} \Phi_{1}\right|+\frac{\lambda_{5}}{2}\left[\left(\Phi_{1}^{\dagger} \tilde{\Phi}_{2}\right)^{2}+\left(\tilde{\Phi}_{2}^{\dagger} \Phi_{1}\right)^{2}\right]
\end{aligned}
$$

in which

$$
\tilde{\Phi}=i \sigma_{2} \Phi^{*}
$$

After incorporating the minimization conditions, the scalar potential parameters can be replaced by physical masses and mixing angles. There are two mixing angles: the angle $\beta=\tan ^{-1} v_{2} / v_{1}$, and the angle $\alpha$, which is the mixing angle of the CP-even Higgs sector. The quantity $\cos (\beta-\alpha)$ is of particular interest in that when $\cos (\beta-\alpha) \rightarrow 0$, the lightest neutral CP-even Higgs boson $h$ behaves like the Higgs boson of the SM, and the additional Higgs bosons decouple (for a comprehensive analysis of the CP-conserving 2HDM in the decoupling limit, see [104]).

Returning to the replacement of the $\lambda_{i}$ by the physical masses and mixing angles, it is convenient to parametrize them in terms of $v$, the $Z_{2}$-breaking potential parameter 
$M$, the Higgs masses $M_{h}, M_{H}, M_{H^{ \pm}}, M_{A}$, and the angles $\alpha$, and $\beta$, as follows:

$$
\begin{aligned}
& \lambda_{1}=\frac{-M^{2} \tan ^{2} \beta+M_{h}^{2} \sin ^{2} \alpha \sec ^{2} \beta+M_{H}^{2} \cos ^{2} \alpha \sec ^{2} \beta}{v^{2}}, \\
& \lambda_{2}=\frac{-M^{2} \cot ^{2} \beta+M_{h}^{2} \cos ^{2} \alpha \csc ^{2} \beta+M_{H}^{2} \sin ^{2} \alpha \csc ^{2} \beta}{v^{2}}, \\
& \lambda_{3}=\frac{-M^{2}+\frac{1}{4}\left(M_{H}^{2}-M_{h}^{2}\right) \sin 2 \alpha \csc 2 \beta+2 M_{H^{ \pm}}^{2}}{v^{2}}, \\
& \lambda_{4}=\frac{M^{2}+M_{A}^{2}-2 M_{H^{ \pm}}^{2}}{v^{2}}, \\
& \lambda_{5}=\frac{M^{2}-M_{A}^{2}}{v^{2}} .
\end{aligned}
$$

In our analysis, we require for simplicity that the heavy physical mass scales are all equivalent, i.e. $M_{H}=M_{A}=M_{H^{ \pm}}$, which serves to ease any tension that would exist with electroweak precision data that prefers a small mass splitting. We see from the above expressions that $\lambda_{3}+\lambda_{4}+\lambda_{5}$, which is what appears in the trilinear scalar couplings of $h$ and $H$ (see e.g. [18] for details), is

$$
\lambda_{3}+\lambda_{4}+\lambda_{5}=\frac{M^{2}+\left(M_{H}^{2}-M_{h}^{2}\right) \csc 2 \beta \sin 2 \alpha}{v^{2}},
$$

and thus is unaffected by the assumption that the heavier Higgs particles are massdegenerate.

We now impose the conditions that the potential maintains perturbative unitarity and is not unbounded from below. As demonstrated in [132 (and discussed in detail in [18]), the conditions to be satisfied for perturbative unitarity are that the following quantities are $\leq 8 \pi$ :

$$
\begin{aligned}
a_{ \pm} & =\frac{3}{2}\left(\lambda_{1}+\lambda_{2}\right) \pm \frac{1}{2} \sqrt{9\left(\lambda_{1}-\lambda_{2}\right)^{2}+\left(2 \lambda_{3}+\lambda_{4}\right)^{2}} \\
b_{ \pm} & =\frac{1}{2}\left(\lambda_{1}+\lambda_{2} \pm \sqrt{\left(\lambda_{1}-\lambda_{2}\right)^{2}+4 \lambda_{4}^{2}}\right) \\
c_{ \pm} & =\frac{1}{2}\left(\lambda_{1}+\lambda_{2} \pm \sqrt{\left(\lambda_{1}-\lambda_{2}\right)^{2}+4 \lambda_{5}^{2}}\right) \\
f_{+} & =\lambda_{3}+2 \lambda_{4}+3 \lambda_{5}, \quad f_{-}=\lambda_{3}+\lambda_{5}, \quad f_{1}=f_{2}=\lambda_{3}+\lambda_{4}, \\
e_{1} & \left.=\lambda_{3}+2 \lambda_{4}-3 \lambda_{5}, \quad e_{2}=2 \lambda_{3}-\lambda_{5}, \quad p_{1}=\lambda_{3}-\lambda_{4}\right),
\end{aligned}
$$


The necessary and sufficient conditions for the potential to remain unbounded from below are [133]:

$$
\begin{gathered}
\lambda_{1} \geq 0, \quad \lambda_{2} \geq 0 \quad, \quad \lambda_{3} \geq-\sqrt{\lambda_{1} \lambda_{2}}, \\
\lambda_{3}+\lambda_{4}-\left|\lambda_{5}\right| \geq-\sqrt{\lambda_{1} \lambda_{2}} .
\end{gathered}
$$

In Fig. 4.2, we show the constraints arising from the requirement of perturbative unitarity in the $\tan \beta$ v. $M_{H}$ and $\tan \beta$ v. $\cos (\beta-\alpha)$ planes for $M / M_{H}=0.8$. We see that the $\tan \beta-\cos (\beta-\alpha)$ plane is particularly instructive for inspecting Higgs couplings. Heavy state masses up to $1 \mathrm{TeV}$ may be possible near $\tan \beta=1$.
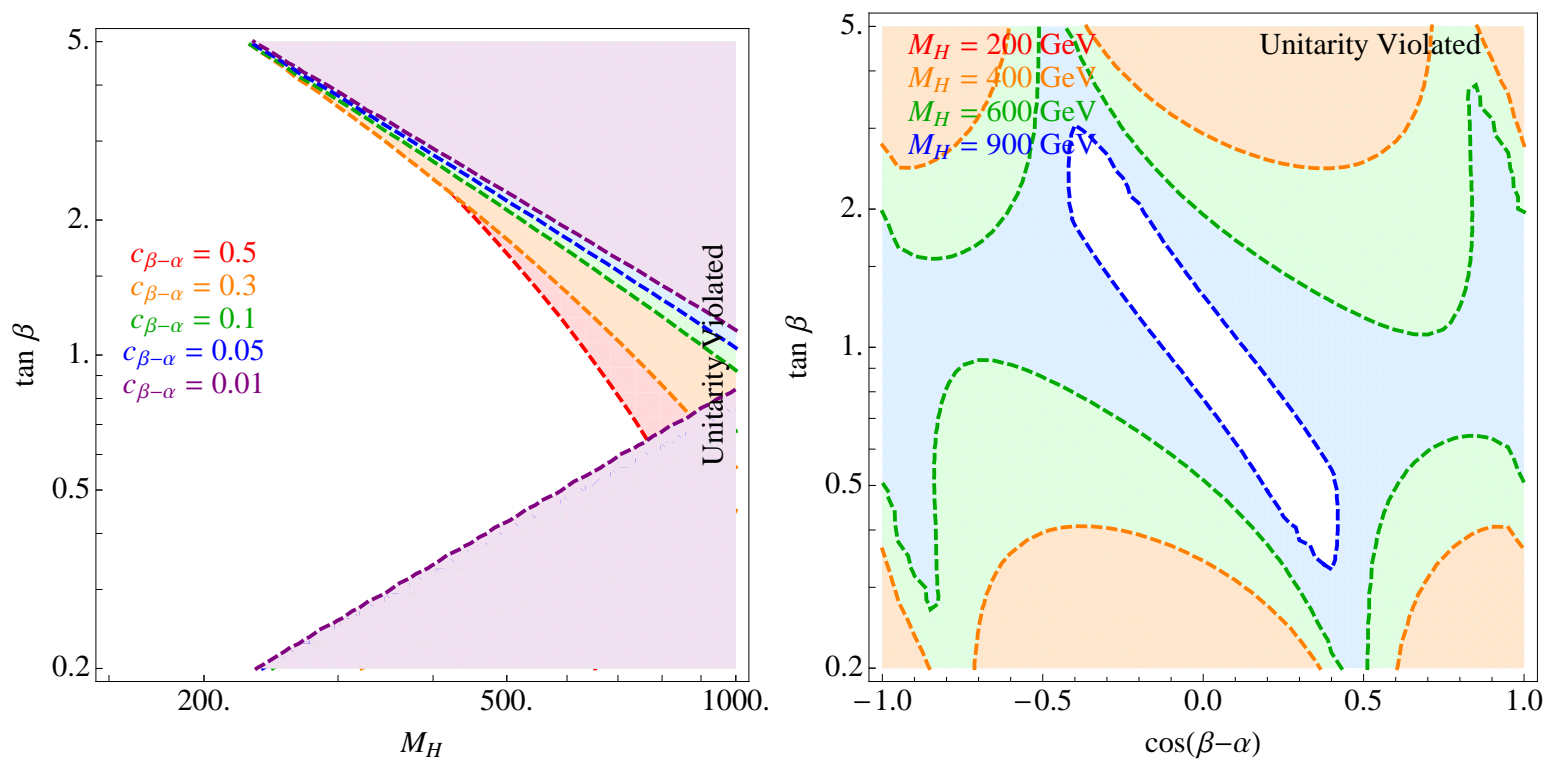

Figure 4.2 Regions that violate perturbative unitarity are shaded in the colors corresponding to the listed values of $\cos (\beta-\alpha)$. The decoupling of the heavy Higgs sector for $\cos (\beta-\alpha) \rightarrow 0$ can allow for a rather large value of $M$.

In Fig. 4.3, we demonstrate how both constraints combine to limit the available ranges of $\tan \beta$ and $M_{H}$, for selected values of $\cos (\beta-\alpha)$ and $M / M_{H}$. For $M / M_{H}=1$, the bounded potential constraint severely limits the available parameter space, while the perturbative unitarity condition is substantially relaxed. For lower values of $M / M_{H}$, the 

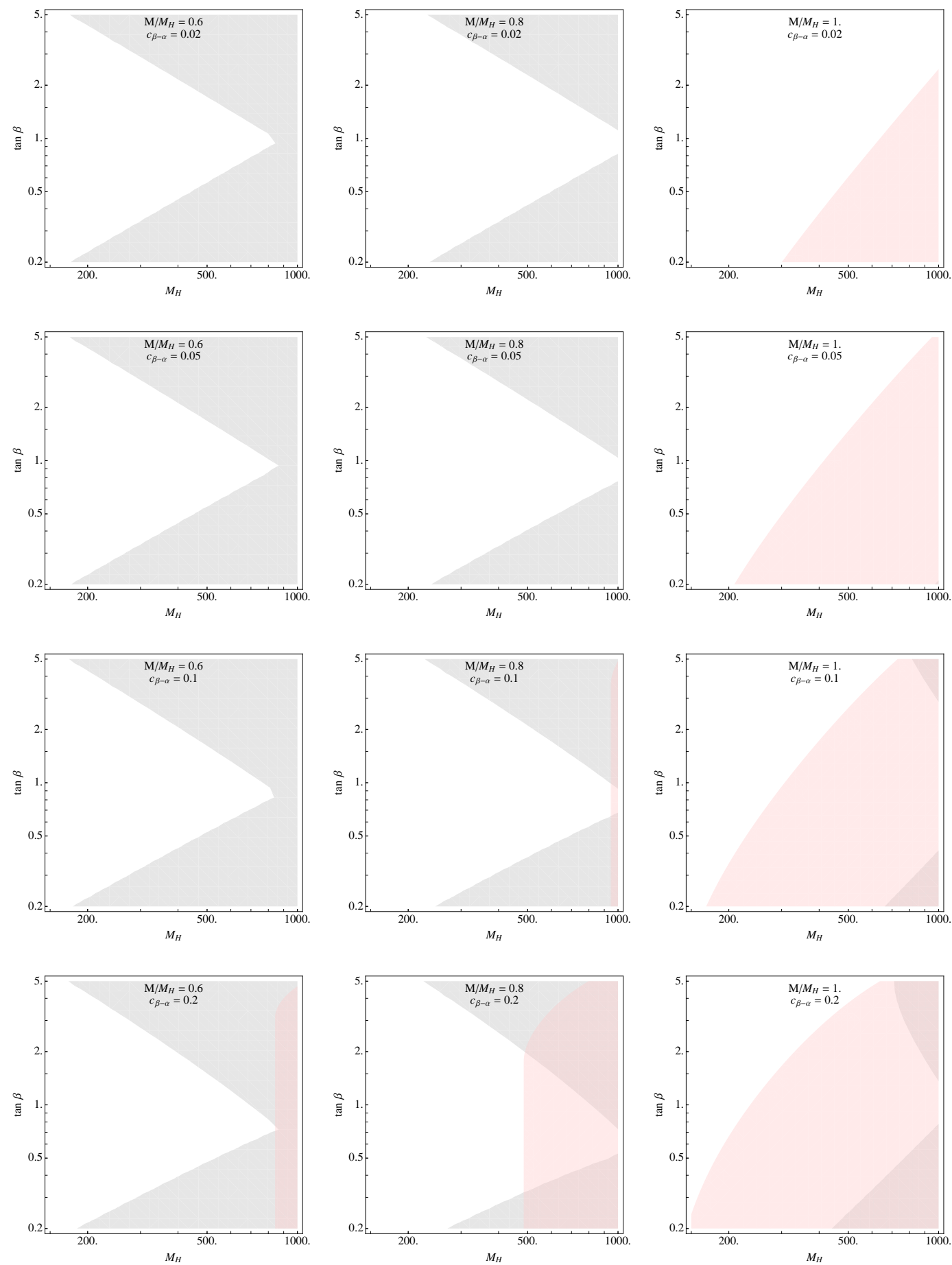

Figure 4.3 Regions that violate perturbative unitarity (gray) and do not have a bounded potential (pink) are shaded for selected values of $\cos (\beta-\alpha)$ and $M / M_{H}$. 
potential constraint is not as severe. For the remainder of this work, we fix $M / M_{H}=0.8$ for illustrative purposes.

\subsubsection{Yukawa couplings}
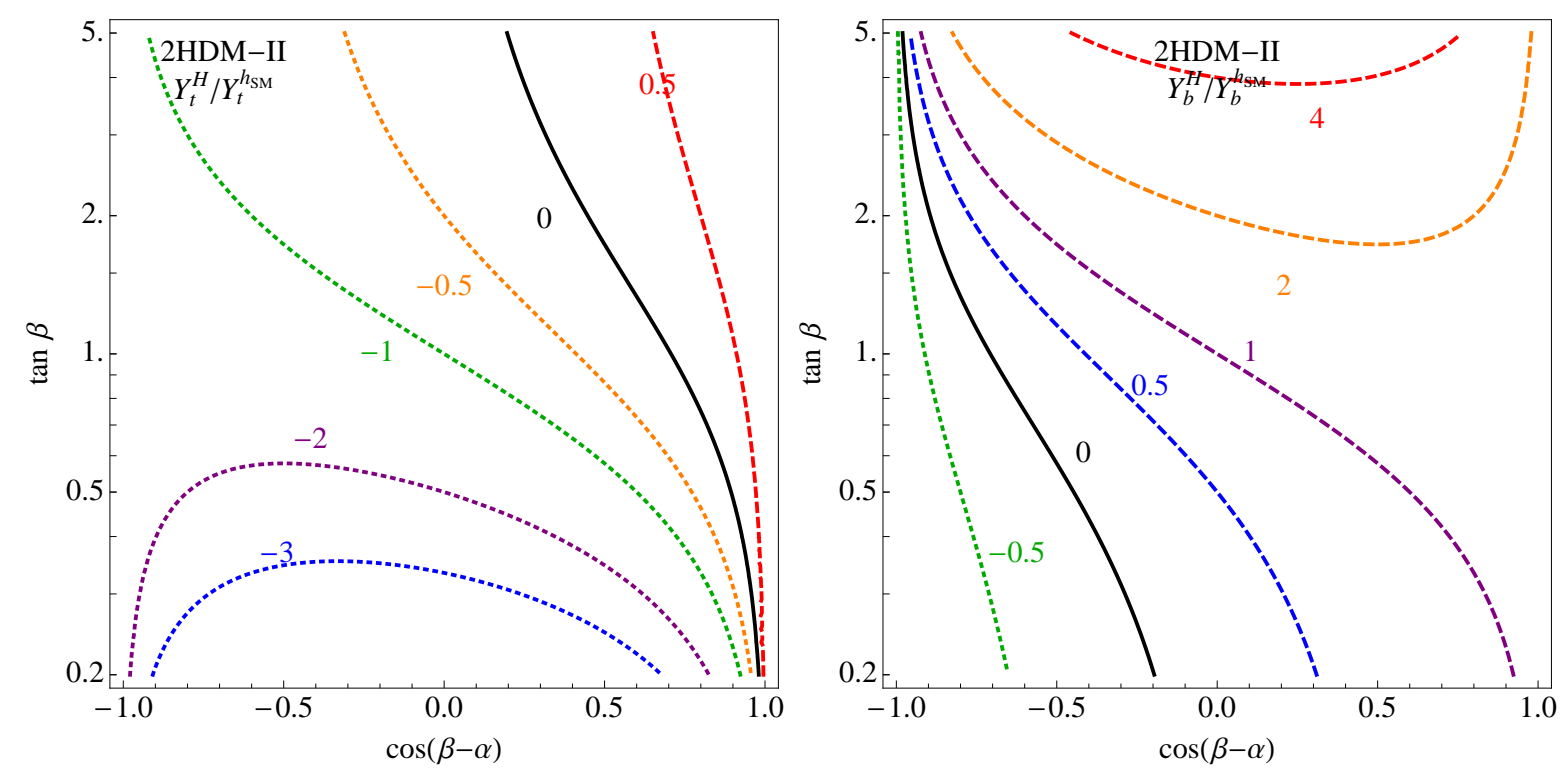

Figure 4.4 Contours of the heavy Higgs Yukawa coupling to $t$ and $b$-quarks in the plane of $\tan \beta$ and $M_{H}$ for selected values of $\cos (\beta-\alpha)$.

For concreteness, we adopt the Yukawa sector of the Type-II 2HDM (the 2HDM-II), which takes the form (see e.g. [109, 110, 111, 112, 113, 104]):

$$
-\mathcal{L}_{\text {Yuk }}=y_{d} \bar{d}_{R} \Phi_{1} Q_{L}-y_{u} \bar{u}_{R} \Phi_{2} Q_{L}+y_{\ell} \bar{\ell}_{R} \Phi_{1} L_{L} .
$$

In Fig. 4.4, we show the Yukawa couplings of the heavy Higgs boson to $t$ and $b$ quarks in this scenario as contours in the $\tan \beta \mathrm{v} \cdot \cos (\beta-\alpha)$ plane.

Within the 2HDM-II, measurements of the $h$ boson couplings with LHC Run-I data constrain the available ranges of $\cos (\beta-\alpha)$ and $\tan \beta$. A number of recent studies have found that the range of $\cos (\beta-\alpha)$ to be no more than 0.1 at $95 \%$ C.L in light of these data [134, 135, 136, 137, 138]. However, some additional freedom can be given in other versions of the 2HDM, such as the Type-I [139] or Lepton specific models [140, 141, 142]. 
A generic Yukawa aligned model with suppressed tree-level FCNCs is also consistent with the LHC data [143, 144, 145, 146].

Complementarity of the gauge couplings forces a limit on the value of $\cos (\beta-\alpha)$ from the vector boson couplings of $h$ alone. We find that the combined ATLAS and CMS Run-I data [99] from vector boson coupling measurements provides a lower limit of $\kappa_{V}=\sin (\beta-\alpha)>0.89$ at the $95 \%$ C.L., which translates to an upper limit of

$$
|\cos (\beta-\alpha)| \lesssim 0.45
$$

In the $2 \mathrm{HDM}$ illustrations provided, these facts should be kept in mind for the larger values of $\cos (\beta-\alpha)$. For the $h$ state, for simplicity, we assume branching fractions consistent with the SM Higgs boson.

\subsubsection{Scalar couplings}

The triscalar coupling, $\lambda^{h h h}$ in the SM takes the value

$$
\lambda_{\mathrm{SM}}^{h h h}=\frac{3 M_{h}^{2}}{v^{2}} .
$$

Recent analyses of measuring this coupling at the LHC via the $h h$ continuum have shown that it may be possible to measure it with an uncertainty of order 30-50\% [147, 148]. Substantial deviations away from the SM value allow a better determination due to interference effects [148].

In the $2 \mathrm{HDM}$, this coupling is altered to

$\lambda^{h h h}=\frac{3 M_{h}^{2}}{2 v} \csc 2 \beta(\cos (3 \alpha-\beta)+3 \cos (\alpha+\beta))-\frac{6 M^{2}}{v} \csc 2 \beta \cos ^{2}(\beta-\alpha) \cos (\alpha+\beta(4.20)$

Expanding in the decoupling limit parameter $\cos (\beta-\alpha) \rightarrow 0$, the deviation of this coupling from its SM value is a second order effect. It can be cast into the form

$$
\lambda^{h h h} \approx \frac{3 M_{h}^{2}}{v^{2}}+\cos ^{2}(\beta-\alpha) \frac{9 M_{h}^{2}-12 M^{2}}{2 v} \approx \lambda_{\mathrm{SM}}^{h h h}\left[1+\cos ^{2}(\beta-\alpha)\left(\frac{3}{2}-\frac{2 M^{2}}{M_{h}^{2}}\right)\right]
$$

in which higher order terms in $\cos (\beta-\alpha)$ have been dropped. 

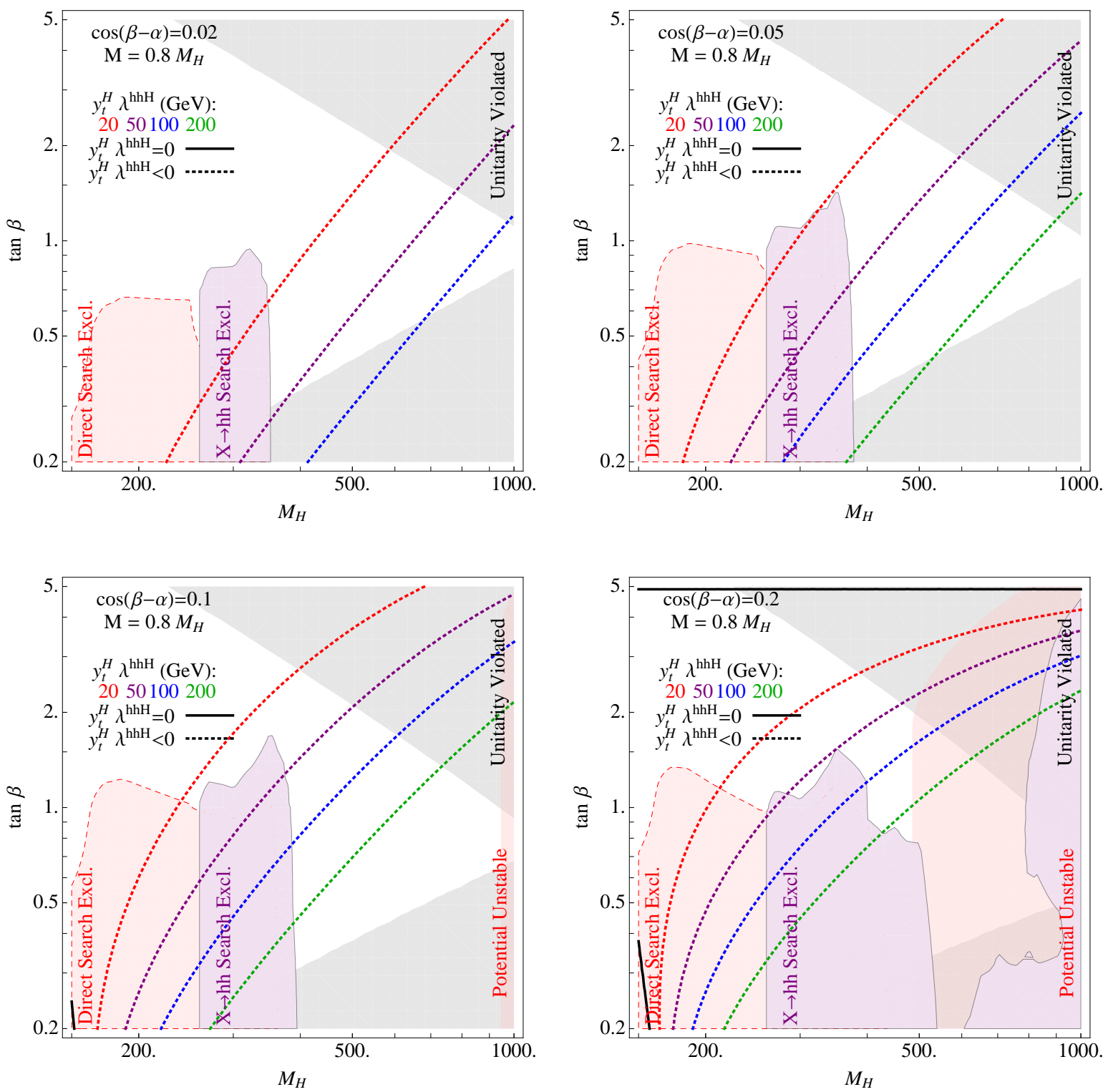

Figure 4.5 Contours of $\lambda^{h h H}$ in the plane of $\tan \beta$ and $M_{H}$ (in $\mathrm{GeV}$ ) for selected values of the decoupling parameter $\cos (\beta-\alpha)$. Included are the unitarity (gray) and vacuum stability (pink) constraints assuming $M=0.8 M_{H}$, the direct search exclusion limits (dashed pink) from CMS [21] and the $h h \rightarrow b \bar{b} \gamma \gamma$ resonance search (purple) [149, 150, 151]. 
The combination $y_{t} \lambda^{h h H}$ is the most relevant for the process of interest. The possible values it may take are shown in Fig. 4.5 for selected values of $\cos (\beta-\alpha)=0.02,0.05,0.1$ and 0.2. We also show the excluded regions from the direct search of $H$ at the LHC [21] via vector boson decays, and from the search for a resonance in the $h h \rightarrow b \bar{b} \gamma \gamma$ final state [149, 150, 151].

The scalar couplings involving the heavy CP-even neutral Higgs that are important for additional search channels are given by

$$
\begin{aligned}
\lambda^{h h H} & =\frac{\cos (\beta-\alpha)}{\sin 2 \beta}\left(\frac{M^{2}(\sin 2 \beta-3 \sin 2 \alpha)+\left(2 M_{h}^{2}+M_{H}^{2}\right) \sin 2 \alpha}{v}\right), \\
\lambda^{h H H} & =\frac{\sin (\beta-\alpha)}{\sin 2 \beta}\left(\frac{M^{2}(\sin 2 \beta+3 \sin 2 \alpha)-\left(M_{h}^{2}+2 M_{H}^{2}\right) \sin 2 \alpha}{v}\right) .
\end{aligned}
$$

As previously discussed, these couplings have no $M_{A}$ and $M_{H^{ \pm}}$dependence (as they depend on the combination $\lambda_{3}+\lambda_{4}+\lambda_{5}$ ), and hence our assumption of heavy Higgs mass degeneracy does not affect these couplings. In the decoupling limit, these expressions take the form

$$
\begin{aligned}
\lambda^{h h H} & \approx \cos (\beta-\alpha) \frac{4 M^{2}-2 M_{h}^{2}-M_{H}^{2}}{v} \\
\lambda^{h H H} & \approx \frac{-2 M^{2}+M_{h}^{2}+2 M_{H}^{2}}{v}+\cos (\beta-\alpha) \frac{2\left(-3 M^{2}+M_{h}^{2}+2 M_{H}^{2}\right) \cot 2 \beta}{v},(
\end{aligned}
$$

neglecting terms of $O\left(\cos (\beta-\alpha)^{2}\right)$. Hence, near the decoupling limit the $h h H$ coupling is suppressed while the $h H H$ coupling persists (see e.g. [104, 152] for discussions). This is shown in Fig. 4.6, which gives the contours of $\mathrm{BF}(H \rightarrow h h)$; additional details of the $H$ decay modes are discussed in Appendix 4.3. The window of $2 m_{h}<M_{H}<2 m_{t}$ in which the $\mathrm{BF}(H \rightarrow h h)$ is quite large and in some cases already ruled out for low $\tan \beta$. In Section 4.5.1, we will see that the discovery potential roughly follows this region, but with a few caveats.

By extracting the $\lambda^{h h h}, \lambda^{h h H}$ and $\lambda^{h H H}$ couplings to some degree of precision, the self-consistency of the scalar model may be tested. More precisely, by measuring the physical masses $M_{h}$ and $M_{H}$ and the heavy Higgs coupling to vector bosons, it is possible to determine whether the expressions given in Eqs. 4.21, 4.22 and 4.23 are self-consistent. 

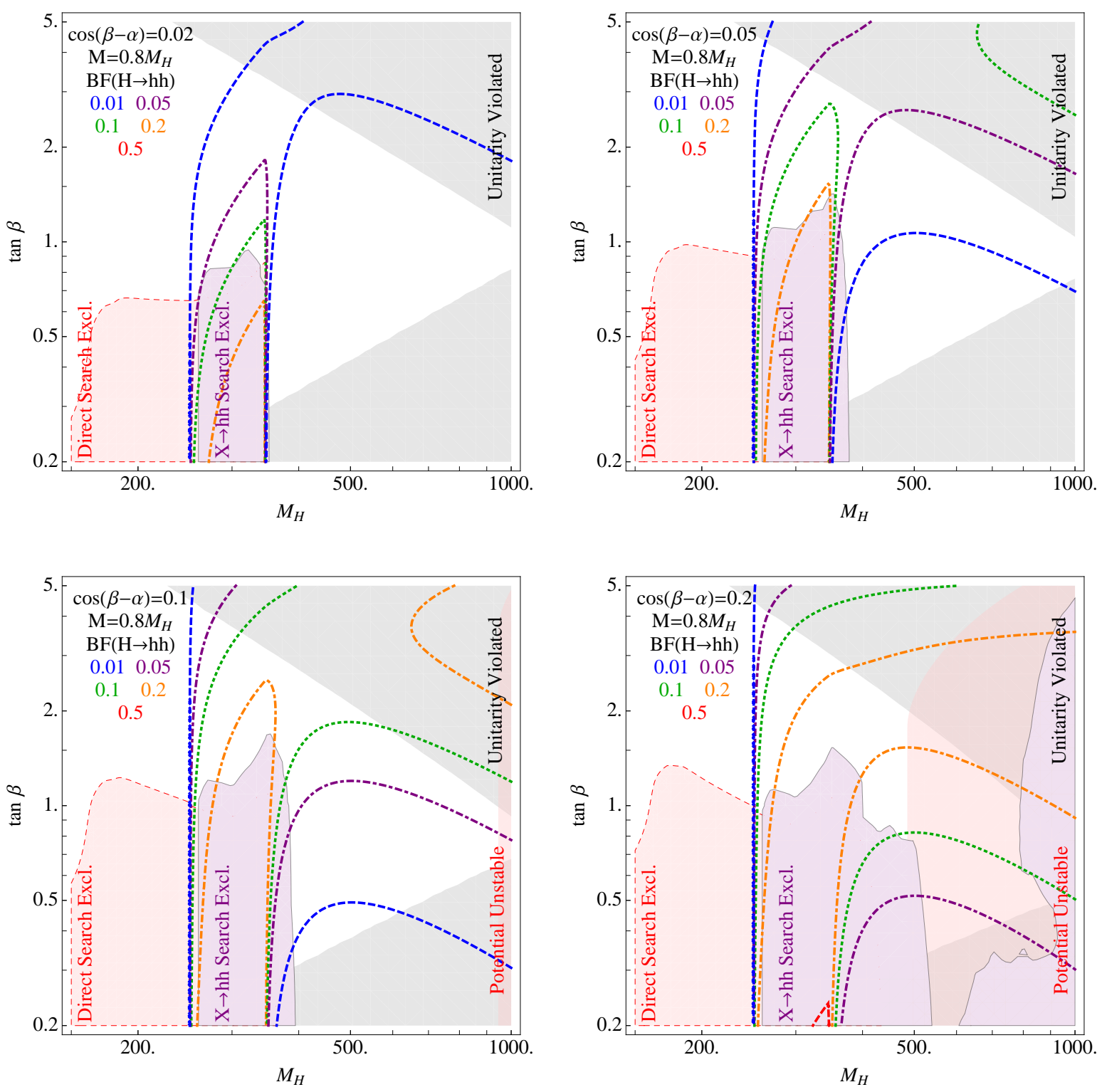

Figure 4.6 Contours of $B F(H \rightarrow h h)$ in the plane of $\tan \beta$ and $M_{H}$ for selected values of $\cos (\beta-\alpha)$. Additional experimental and theoretical constraints are shown as in Fig. 4.5. 
In the subsequent analyses, we will refer to three benchmark points that help elucidate the discovery potential of each channel. The points are summarized in Table 4.1 . Benchmark point A will illustrate the viability of the $H \rightarrow h h \rightarrow b \bar{b} \gamma \gamma$ channel, point B the $h h / h H \rightarrow b \bar{b} \gamma \gamma$ and $b \bar{b} b \bar{b}$ channels, and point $\mathrm{C}$ the $h H \rightarrow t \bar{t} b \bar{b}$ channel.

\subsection{Heavy Higgs Branching Fractions}

We calculate the branching fractions of the Heavy Higgs via the expected SM-like partial widths

$$
\begin{aligned}
\Gamma_{H \rightarrow V V} & =\cos (\beta-\alpha)^{2} \Gamma_{H \rightarrow V V}^{\mathrm{SM}}, \\
\Gamma_{H \rightarrow b \bar{b}} & =\left(y_{t}^{H} / y_{t}^{h_{\mathrm{SM}}}\right)^{2} \Gamma_{H \rightarrow b \bar{b}}^{\mathrm{SM}}, \\
\Gamma_{H \rightarrow t \bar{t}} & =\left(y_{b}^{H} / y_{b}^{h_{\mathrm{SM}}}\right)^{2} \Gamma_{H \rightarrow t \bar{t}}^{\mathrm{SM}}, \\
\Gamma_{H \rightarrow \tau^{+} \tau^{-}} & =\left(y_{\tau}^{H} / y_{\tau}^{h_{\mathrm{SM}}}\right)^{2} \Gamma_{H \rightarrow \tau^{+} \tau^{-}}^{\mathrm{SM}} .
\end{aligned}
$$

where $\Gamma^{\mathrm{SM}}$ indicates the SM-like partial width with $M_{h_{\mathrm{SM}}}=M_{H}$. For these calculations, we neglect the partial decays to $\gamma \gamma$ and $g g$ and light quarks as they're negligible for the cases we consider. We calculate the SM-like Higgs partial widths with the HDECAY package [179]. The heavy Higgs partial width to the SM-like Higgs boson at $m_{h}=125$ $\mathrm{GeV}$ is given by

$$
\Gamma_{H \rightarrow h h}=\frac{\left(\lambda^{h h H}\right)^{2}}{32 \pi M_{H}} \sqrt{1-\frac{4 m_{h}^{2}}{M_{H}^{2}}} .
$$

The total Higgs boson width is calculated according to the sum of the respective partial widths

$$
\Gamma_{H}=\Gamma_{H \rightarrow V V}+\Gamma_{H \rightarrow b \bar{b}}+\Gamma_{H \rightarrow t \bar{t}}+\Gamma_{H \rightarrow \tau^{+} \tau^{-}}+\Gamma_{H \rightarrow h h},
$$

leading to the branching fractions that are calculated in the usual way

$$
\mathrm{BF}(H \rightarrow X \bar{X})=\frac{\Gamma_{H \rightarrow X X}}{\Gamma_{H}},
$$



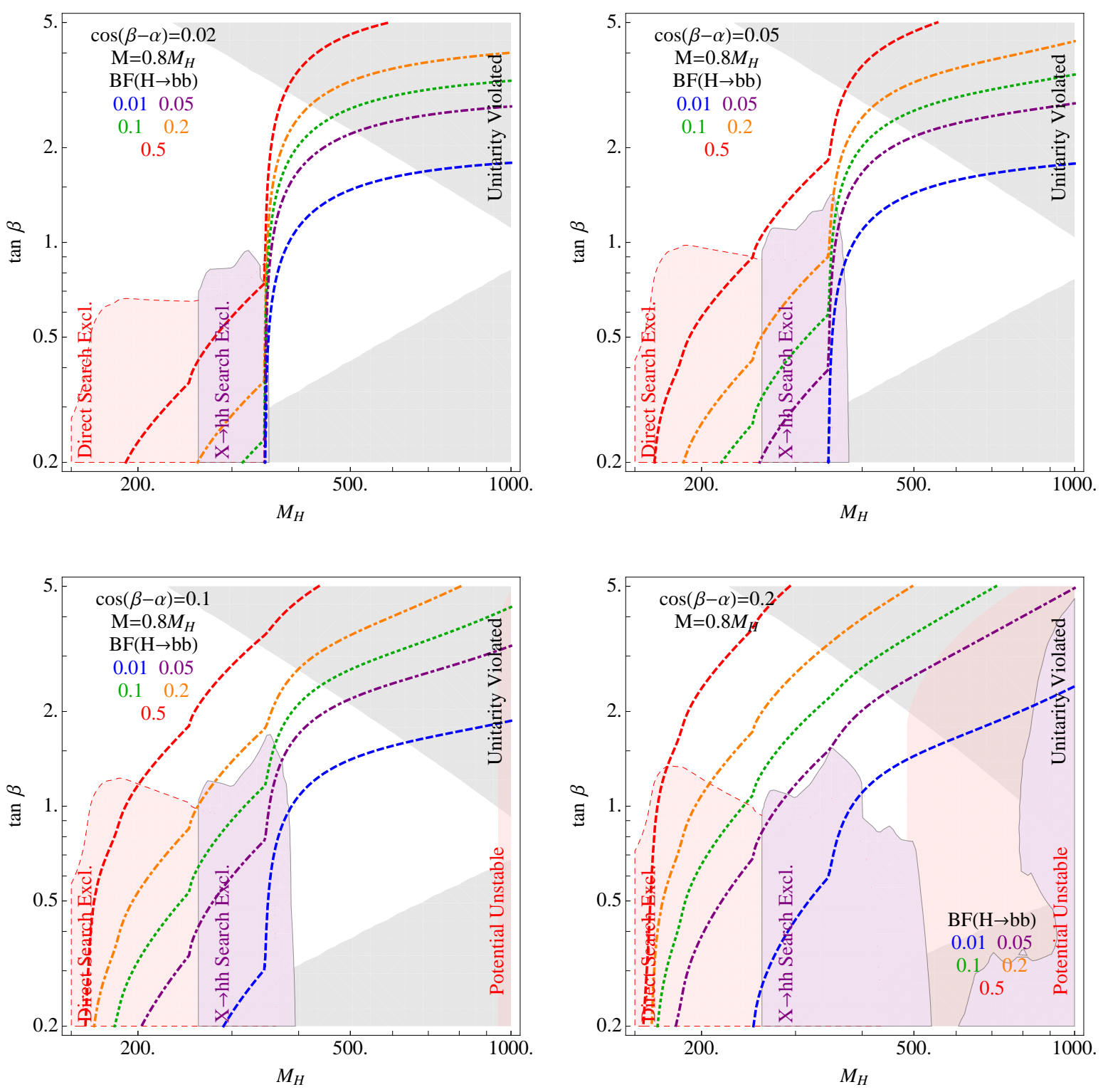

Figure 4.7 Contours of $B F(H \rightarrow b \bar{b})$ in the plane of $\tan \beta$ and $M_{H}$ for selected values of $\cos (\beta-\alpha)$. Additional experimental and theoretical constraints are shown as in Fig. 4.5.

where $X X=V V, b \bar{b}, t \bar{t}, \tau^{+} \tau^{-}$and $h h$. We list in Figs. 4.7, 4.8, and 4.9 the contours of branching fractions in the selected parameter planes for $b \bar{b}, t \bar{t}$ and $V V$, respectively. The branching fraction to $h h$ is shown in Fig. 4.6 in Section 4.2 .2 . 

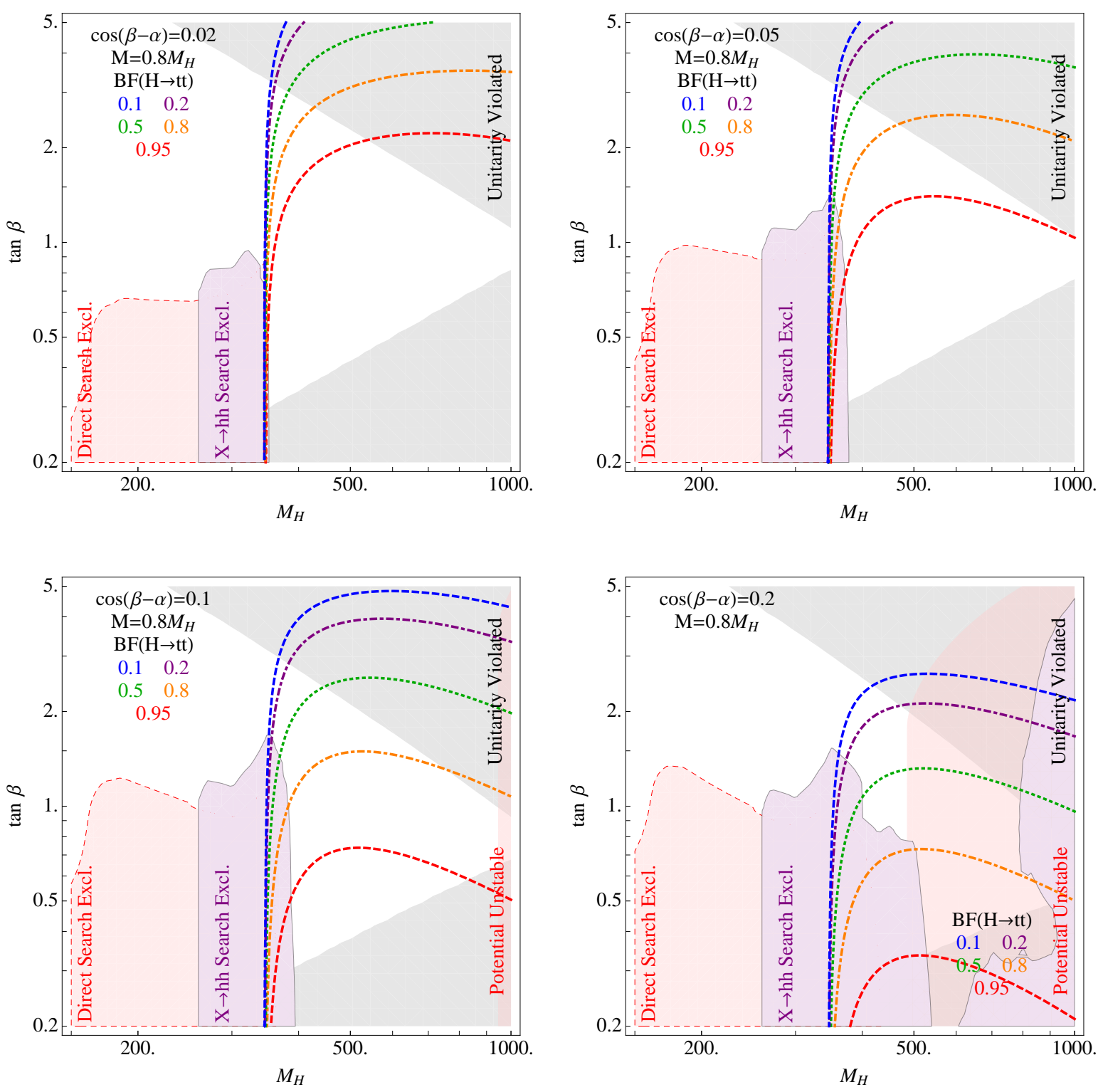

Figure 4.8 Contours of $B F(H \rightarrow t \bar{t})$ in the plane of $\tan \beta$ and $M_{H}$ for selected values of $\cos (\beta-\alpha)$. Additional experimental and theoretical constraints are shown as in Fig. 4.5.

\subsection{Higgs Pair Production Cross Section}

Pairs of neutral Higgs bosons can be generated through two different loop processes (depicted in Fig. 4.1): (i) the triangle diagram where an s-channel Higgs boson decays into two Higgs bosons and (ii) the box diagram where annihilation of two gluons through 

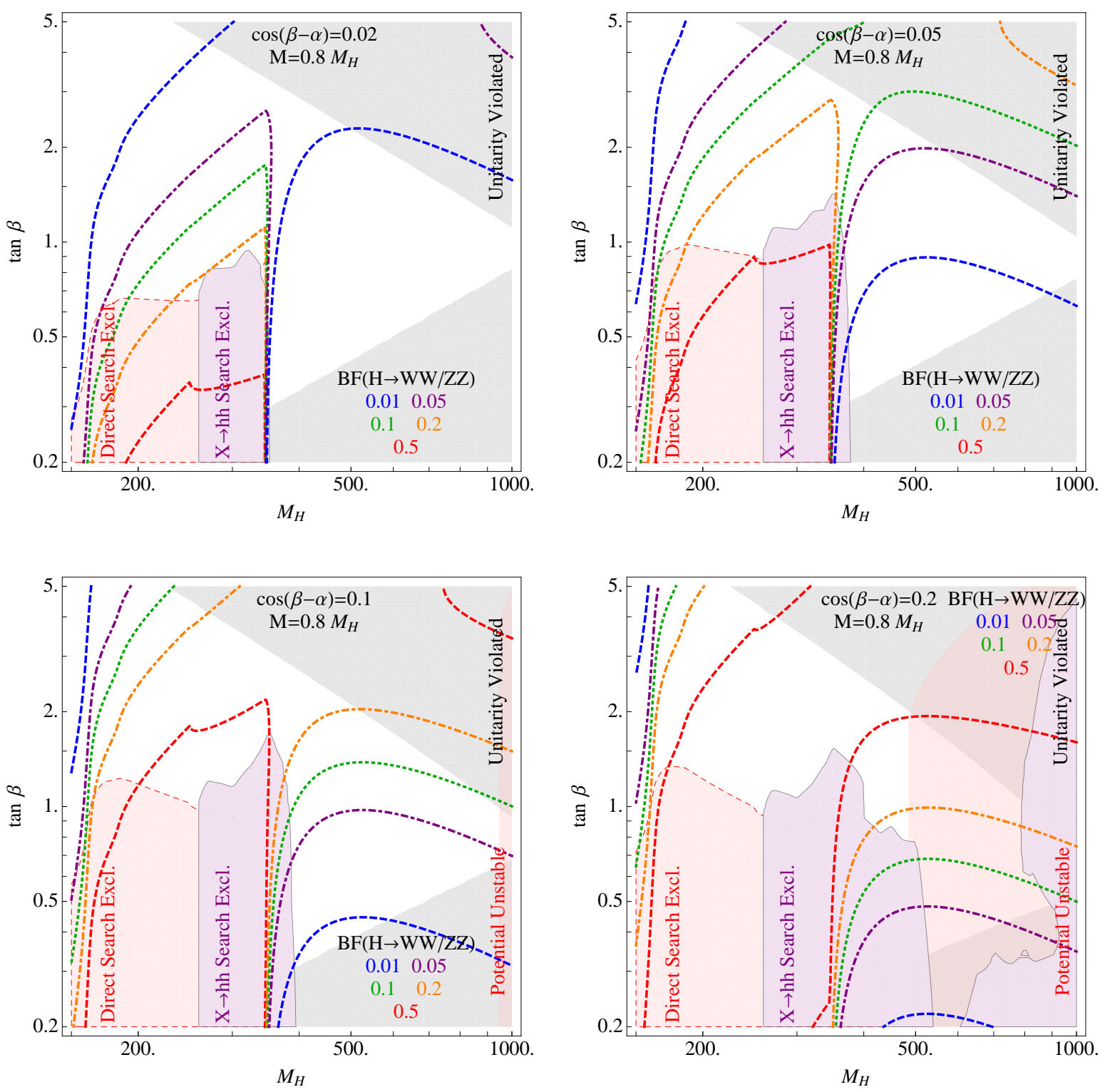

Figure 4.9 Contours of $B F\left(H \rightarrow W W^{(*)} / Z Z^{(*)}\right)$ in the plane of $\tan \beta$ and $M_{H}$ for selected values of $\cos (\beta-\alpha)$. Additional experimental and theoretical constraints are shown as in Fig. 4.5 .

a square loop produces a Higgs boson pair. The exact expressions for these one-loop diagrams with generic internal/external Higgs bosons (as well as generic heavy quarks) were first computed in Ref. [153]. We have independently confirmed the expressions for 
the loop diagrams and we present them here just for completeness. Readers interested in the finer details are referred to Sections 3 and 4 of Ref. [153].

First, let us introduce some notation. Denoting the intial-state gluon momenta as $p_{a, b}$ and the final-state Higgs boson momenta as $p_{j, k}$, the Mandelstam invariants are given by:

$$
\hat{s}=\left(p_{a}+p_{b}\right)^{2} ; \hat{t}=\left(p_{a}-p_{j}\right)^{2} ; \hat{u}=\left(p_{a}-p_{k}\right)^{2}
$$

It is also useful to define the quantities

$$
\begin{gathered}
S=\hat{s} / m_{Q}^{2} ; T=\hat{t} / m_{Q}^{2} ; U=\hat{u} / m_{Q}^{2}, \\
\rho_{j}=M_{j}^{2} ; \rho_{k}=M_{k}^{2} ; \tau_{Q}=4 / S, \\
T_{1}=T-\rho_{j}, U_{1}=U-\rho_{j}, T_{2}=T-\rho_{k}, U_{2}=U-\rho_{k} .
\end{gathered}
$$

In the discussion to follow, we will reduce all tensor integrals to scalar ones. The pertinent three- and four-point scalar integrals can be written as

$$
\begin{aligned}
C_{\ell m} & =\int \frac{d^{4} k}{(2 \pi)^{4}} \frac{1}{\left(k^{2}-m_{Q}^{2}\right)\left(\left(k+p_{\ell}\right)^{2}-m_{Q}^{2}\right)\left(\left(k+p_{\ell}+p_{m}\right)^{2}-m_{Q}^{2}\right)}, \\
D_{\ell m n} & =\int \frac{d^{4} k}{(2 \pi)^{4}} \frac{1}{\left(k^{2}-m_{Q}^{2}\right)\left(\left(k+p_{\ell}\right)^{2}-m_{Q}^{2}\right)\left(\left(k+p_{\ell}+p_{m}\right)^{2}-m_{Q}^{2}\right)\left(\left(k+p_{\ell}+p_{m}+p_{n}\right)^{2}-m_{Q}^{2}\right)},
\end{aligned}
$$

in which $\ell, m, n$ label momenta entering the loop.

The matrix element of the triangle diagram can be written in terms of a "coupling" $C_{\triangle}$ and a form factor $F_{\triangle}$ as:

$$
\mathcal{M}_{\triangle}=\frac{G_{F} \alpha_{s} \hat{s}}{2 \sqrt{2} \pi} C_{\triangle} F_{\triangle} A_{1, \mu \nu} \epsilon_{a}^{\mu} \epsilon_{b}^{\nu} \delta_{a b},
$$

in which the tensor structure $A_{1}^{\mu \nu}$ is:

$$
A_{1}^{\mu \nu}=g^{\mu \nu}-\frac{p_{a}^{\nu} p_{b}^{\mu}}{\left(p_{a} \cdot p_{b}\right)} .
$$


The coupling factor can be expressed as:

$$
C_{\triangle}=\sum_{i=h, H} C_{\triangle}^{i}
$$

with:

$$
C_{\triangle}^{i}=\lambda_{H_{i} H_{j} H_{k}} \frac{M_{Z}^{2}}{\hat{s}-M_{H_{i}}^{2}+i M_{i} \Gamma_{H_{i}}} y_{H_{i} Q \bar{Q}},
$$

in which $y_{H_{i} Q \bar{Q}}$ denotes the heavy quark Yukawa coupling to $H_{i}$. The form factor $F_{\triangle}$ can be computed in closed form, and is given by

$$
F_{\triangle}=\frac{2}{S}\left[2+(4-S) m_{Q}^{2} C_{j k}\right]=\tau_{Q}\left[1+\left(1-\tau_{Q}\right) f\left(\tau_{Q}\right)\right]
$$

in which

$$
f\left(\tau_{Q}\right)=\left\{\begin{array}{cc}
\arcsin ^{2} \frac{1}{\sqrt{\tau_{Q}}} & \tau_{Q} \geq 1 \\
-\frac{1}{4}\left[\log \frac{1+\sqrt{1-\tau_{Q}}}{1-\sqrt{1-\tau_{Q}}}-i \pi\right]^{2} & \tau_{Q}<1 .
\end{array}\right.
$$

The matrix element for the box diagrams can be written in terms of a coupling factor $C_{\square}$ and two gauge-invariant form factors $F_{\square}$ and $G_{\square}$ as:

$$
\mathcal{M}_{\square}=\frac{G_{F} \alpha_{s} \hat{s}}{2 \sqrt{2} \pi} C_{\square}\left(F_{\square} A_{1, \mu \nu}+G_{\square} A_{2, \mu \nu}\right) \epsilon_{a}^{\mu} \epsilon_{b}^{\nu} \delta_{a b},
$$

where $A_{1, \mu \nu}$ is given in Eq. (4.34) and the other tensor structure takes the form

$$
A_{2}^{\mu \nu}=g^{\mu \nu}+\frac{p_{j}^{2} p_{a}^{\nu} p_{b}^{\mu}}{\left.p_{T}^{(} p_{a} \cdot p_{b}\right)}-\frac{2\left(p_{b} \cdot p_{j}\right) p_{a}^{\nu} p_{j}^{\mu}}{\left.p_{T}^{(} p_{a} \cdot p_{b}\right)}-\frac{2\left(p_{a} \cdot p_{j}\right) p_{b}^{\mu} p_{j}^{\nu}}{\left.p_{T}^{(} p_{a} \cdot p_{b}\right)}+\frac{2 p_{j}^{\mu} p_{j}^{\nu}}{p_{T}^{2}}
$$

with

$$
p_{T}^{2}=2 \frac{\left(p_{a} \cdot p_{j}\right)\left(p_{b} \cdot p_{j}\right)}{\left(p_{a} \cdot p_{b}\right)}-p_{c}^{2} .
$$

The advantage of writing the amplitude in terms of $A_{1,2}^{\mu \nu}$ is that it greatly simplifies the calculation of the matrix-element-squared, since

$$
A_{1} \cdot A_{2}=0 \text { and } A_{1} \cdot A_{1}=A_{2} \cdot A_{2}=2 .
$$


The coupling for the box diagrams is just the product of the two Yukawa couplings of the heavy quark to the two Higgs bosons

$$
C_{\square}=y_{H_{j} Q \bar{Q}} y_{H_{k} Q \bar{Q}}
$$

while the form factors $F_{\square}$ and $G_{\square}$ are given by

$$
\begin{aligned}
F_{\square} & =\frac{1}{S^{2}}\left\{4 S+8 S m_{Q}^{2} C_{a b}-2 S m_{Q}^{4}\left(S+\rho_{j}+\rho_{k}-8\right)\left(D_{a b j}+D_{b a j}+D_{a j b}\right)\right. \\
& +\left(\rho_{j}+\rho_{k}-8\right) m_{Q}^{2}\left[T_{1} C_{a j}+U_{1} C_{b j}+U_{2} C_{a k}+T_{2} C_{b k}-m_{Q}^{2}\left(T U-\rho_{j} \rho_{k}\right) D_{a j b}(4.44)\right.
\end{aligned}
$$

and

$$
\begin{aligned}
G_{\square}= & \frac{1}{S\left(T U-\rho_{j} \rho_{k}\right)}\left\{m_{Q}^{2}\left(T^{2}+\rho_{j} \rho_{k}-8 T\right)\left[S C_{a b}+T_{1} C_{a j}+T_{2} C_{b k}-S T m_{Q}^{2} D_{b a j}\right]\right. \\
& +m_{Q}^{2}\left(U^{2}+\rho_{j} \rho_{k}-8 U\right)\left[S C_{a b}+U_{1} C_{b j}+U_{2} C_{a k}-S U m_{Q}^{2} D_{a b j}\right] \\
& -m_{Q}^{2}\left(T^{2}+U^{2}-2 \rho_{j} \rho_{k}\right)(T+U-8) C_{j k} \\
& \left.-2 m_{Q}^{4}(T+U-8)\left(T U-\rho_{j} \rho_{k}\right)\left(D_{a b j}+D_{b a j}+D_{a j b}\right)\right\}
\end{aligned}
$$

The differential cross section (averaging/summing over initial/final state spins and colors) then takes the following form:

$$
\frac{d \hat{\sigma}\left(g g \rightarrow H_{j} H_{k}\right)}{d \hat{t}}=\frac{G_{F}^{2} \alpha_{s}^{2}}{256(2 \pi)^{3}}\left[\left|\left(C_{\triangle} F_{\triangle}+C_{\square} F_{\square}\right)\right|^{2}+\left|C_{\square} G_{\square}\right|^{2}\right],
$$

To obtain the total parton-level cross section, this expression is integrated over the scattering angle of one of the Higgs bosons. Finally, to convert the parton-level cross section to the proton-proton cross section, we convolute the former with the PDFs for two gluons and integrate over the momentum fraction of the gluons. For the parton distributions, we use CTEQ 6L1.

\subsection{Light Higgs pair production simulation}

The leading order (LO) matrix elements of the $h h$ subprocesses in Fig. 4.1 are known [153, 154, 155, 156, 157]. We generate signal events by incorporating the loop amplitudes 
directly into MADGRAPH [82], and we include the NNLO K-factor of 2.27 for 14 $\mathrm{TeV}$ [158, 159, 18, 160, 161, 162, 163]. We note that in principle, the resonant production can shift the overall K-factor as the ratio $\sigma_{N N L O} / \sigma_{L O}$ can be $\sqrt{s}$ dependent. However, since the K-factor has not been given for this process, we adopt the SM value and assume any shift induced by the $H$ resonance is small. We show the cross section contours of $p p \rightarrow h h$ with the $H \rightarrow h h$ resonance in Fig. 4.10 .

The $p p \rightarrow h h$ cross section can be shifted dramatically away from its SM value by the presence of an extended Higgs sector [164, 165, 166, 167, 168]. The relative competition of the diagrams in Fig. 4.1 strongly impacts the kinematic distributions with the most apparent coming from the resonant $g g \rightarrow H \rightarrow h h$ diagram. Here, if $M_{H}>2 m_{h}$, the resonance can become prominent, overwhelming the continuum from the $g g \rightarrow h h$ box and $g g \rightarrow h^{*} \rightarrow h h$ diagrams, seen as the large cross section in the $250-350$ GeV range, above which, the $H \rightarrow t \bar{t}$ branching fraction dominates.

We note that the sign of the combination $y_{t}^{H} \lambda^{h h H}$ determines the shape of the distribution due to the interference with the continuum diagrams. In principle, measuring the $H \rightarrow h h$ lineshape can determine the sign of $y_{t}^{H} \lambda^{h h H}$, further constraining the model. A simple counting of events above and below resonance will provide a handle on the sign of the coupling combination, while more sophisticated fits including the matrix elements are possible, as has been done in the continuum case [148]. For sufficiently heavy $H$, the lower energy $M_{h h}$ distribution converges to the SM expectation. We explore model independent resonant production of $h \mathrm{~h}$ in more detail in Ref. [164].

Each final state Higgs boson in these events is decayed in the narrow width approximation to SM Higgs decay modes. There are a number of potential final states for the Higgs pair, but most suffer suppression due to small SM branching fractions [128]. As noted in Ref. [148, the $b \bar{b} \tau^{+} \tau^{-}$channel is swamped by the reducible background of $b \bar{b} j j$ where both light flavored jets fake a $\tau$. While the fake rate is in the range of $1-3 \%$, the total cross section of $b \bar{b} j j$ is at the $\mu b$ level. Moreover, we neglect the $b \bar{b} W^{+} W^{-}$channel due to a small SM significance [169]. The $b \bar{b} b \bar{b}$ channel also suffers from a large QCD 

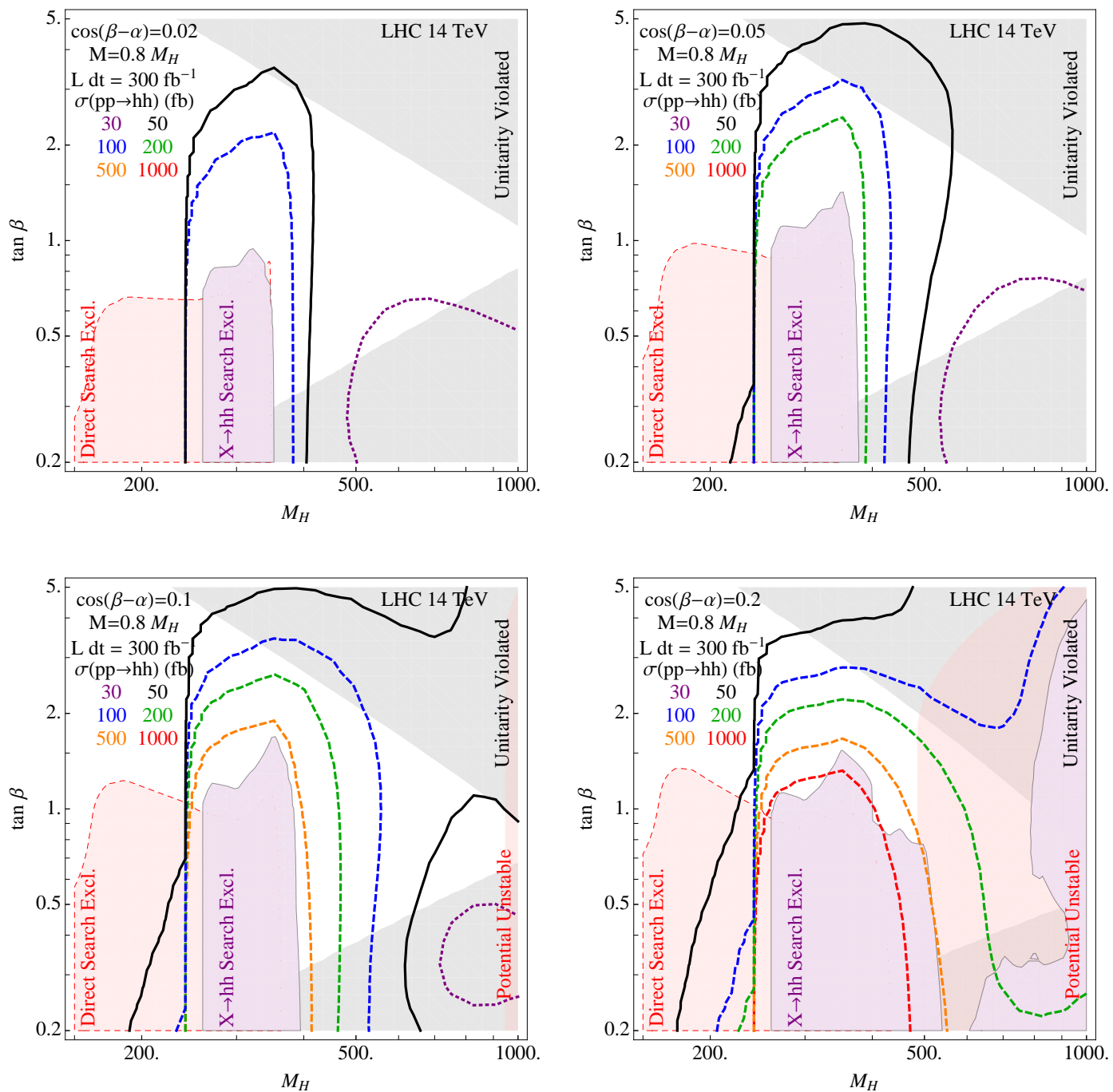

Figure 4.10 Contours of $\sigma(p p \rightarrow h h)$ in the plane of $\tan \beta$ and $M_{H}$ for selected values of $\cos (\beta-\alpha)$. Additional experimental and theoretical constraints are shown as in Fig. 4.5 . 
background, and would only be viable with the use of jet substructure techniques [170]. Therefore, we concentrate on the analysis of the $b \bar{b} \gamma \gamma$ channel for the resonant production of $h h$. Ref. [171], exploring the same channel, appeared while this work was in preparation.

\subsubsection{The $h h \rightarrow b \bar{b} \gamma \gamma$ channel}

We simulate the pertinent backgrounds for the $b \bar{b} \gamma \gamma$ channel. The irreducible background includes the following production modes:

$$
\begin{aligned}
& p p \rightarrow b \bar{b} \gamma \gamma \\
& p p \rightarrow Z+h \rightarrow b \bar{b}+\gamma \gamma
\end{aligned}
$$

while the reducible backgrounds include

$$
\begin{aligned}
& p p \rightarrow t \bar{t}+h \rightarrow b \ell^{+} \nu \bar{b} \ell^{-} \bar{\nu}+\gamma \gamma \quad\left(\ell^{ \pm} \text {missed }\right), \\
& p p \rightarrow b \bar{b}+j j \rightarrow b \bar{b}+\gamma \gamma \quad(j \rightarrow \gamma) .
\end{aligned}
$$

We assume a photon tagging rate of $85 \%$ and a jet to photon fake rate of $\epsilon_{j \rightarrow \gamma}=$ $1.2 \times 10^{-4}[172$. We have determined the additional reducible backgrounds of $j j \gamma \gamma$ and $c \bar{c} \gamma \gamma$ are subdominant, therefore they are not included in this analysis.

To account for $b$ jet tagging efficiencies, we assume a $b$-tagging rate of $70 \%$ for $b$ quarks with $p_{T}>30 \mathrm{GeV}$ and $\left|\eta_{b}\right|<2.4$ consistent with multivariate tagging suggested for the LHC luminosity upgrade [173]. We also apply a mistagging rate for charm-quarks as

$$
\epsilon_{c \rightarrow b}=10 \% \quad \text { for } \quad p_{T}(c)>50 \mathrm{GeV},
$$

while the mistagging rate for a light quark is:

$$
\begin{array}{rlrl}
\epsilon_{u, d, s, g \rightarrow b} & =2 \% & \text { for } & p_{T}(j)>250 \mathrm{GeV} \\
\epsilon_{u, d, s, g \rightarrow b}=0.67 \% & \text { for } & p_{T}(j)<100 \mathrm{GeV} .
\end{array}
$$

Over the range $100 \mathrm{GeV}<p_{T}(j)<250 \mathrm{GeV}$, we linearly interpolate the fake rates given above [174. With pile-up the rejection rate is expected to worsen by up to $20 \%$ [173]. 
Finally, we model detector resolution effects by smearing the final state energy according to

$$
\frac{\delta E}{E}=\frac{a}{\sqrt{E}} \oplus b
$$

where we take $a=50 \%$ and $b=3 \%$ for jets and $a=10 \%$ and $b=0.7 \%$ for photons.

We apply a multi-variate analysis (MVA) which relies on relevant kinematic variables. We begin with low level cuts, requiring two $b$-tags and two $\gamma$-tags and no tagged charged leptons, with separation of $\Delta R_{\gamma \gamma}, \Delta R_{b \bar{b}}, \Delta R_{b \gamma}>0.4$. The value $\Delta R_{a b}=$ $\sqrt{\left(\phi_{a}-\phi_{b}\right)^{2}+\left(\eta_{a}-\eta_{b}\right)^{2}}$ is the separation of two objects in the $\eta-\phi$ plane. We further require $p_{T}(b, \gamma)>30 \mathrm{GeV}$ and $\left|\eta_{b, \gamma}\right|<2.4$.

We define a window within which the MVA will analyze events. This window has the Higgs boson reconstructed in the $b \bar{b}$ and $\gamma \gamma$ channels according to:

$$
\begin{aligned}
\left|M_{b \bar{b}}-M_{h}\right| & <20 \mathrm{GeV} \\
\left|M_{\gamma \gamma}-M_{h}\right| & <10 \mathrm{GeV}
\end{aligned}
$$

We extend our analysis to include multiple variables simultaneously. This allows one to in essence blend cuts together rather than perform a hard cut on a kinematic distribution. We form a discriminant based on a set of observables which include:

$$
\mathcal{O}=\left\{M_{b \bar{b} \gamma \gamma}, M_{b \bar{b}}, M_{\gamma \gamma}, p_{T}(b \bar{b}), p_{T}(\gamma \gamma), \Delta R_{b \bar{b}}, \Delta R_{\gamma \gamma}, \Delta \eta_{\gamma \gamma}, \Delta \eta_{b \bar{b}}\right\}
$$

The discriminant is then constructed by the ratio

$$
\mathcal{D}=\frac{S(\mathcal{O})}{S(\mathcal{O})+A B(\mathcal{O})}
$$

in which $S(\mathcal{O})$ and $B(\mathcal{O})$ are the normalized differential cross sections in the observable space $\mathcal{O}$. These differential cross sections are estimated via event generation. The discriminator is evaluated for an event sample, yielding a value close to 1 for signal-like events and close to 0 for background-like events. For the particular choice of $A=N_{B} / N_{S}$, the discriminant gives the probability of an event being signal [175]. A cut may be placed on the value of $\mathcal{D}$, thereby selecting a relatively high signal event sample. Such a 
multivariate discriminator can offer similar sensitivity that the matrix-element, or neural network methods allow [176].

In practice, we apply a simplified version of the discriminant in which we ignore the correlations among the variables. With limited statistics, this allows a more efficient construction of the discriminator, defined as

$$
\mathcal{D}=\frac{S\left\{\mathcal{O}_{i}\right\}}{S\left\{\mathcal{O}_{i}\right\}+B\left\{\mathcal{O}_{i}\right\}},
$$

where $\left\{\mathcal{O}_{i}\right\}$ is the combinatorial subset of observables $\mathcal{O}$ that go into the multivariate discriminant. In the MVA results that follow, further optimization may be done by including the correlations between observables, but we adopt this uncorrelated approach for simplicity. We define the level of statistical significance, $\mathcal{S}$, according to [177]

$$
\mathcal{S}=2(\sqrt{S+B}-\sqrt{B})
$$

in which $S$ and $B$ are the number of signal and background events surviving cuts. We maximize $\mathcal{S}$ by varying the cut on the discriminator, $\mathcal{D}_{\text {cut }}$, which minimizes the choice of $A$ in Eq. 4.58 ,

In Fig. 4.11, we show the luminosity required to obtain $5 \sigma$ discovery at the LHC. We find that generally these contours follow the shape of the $h h \rightarrow b \bar{b}+\gamma \gamma$ resonance excluded region (shaded in purple) with Run-I data. The contour with $\sqrt{s}=14 \mathrm{TeV}$ and $30 \mathrm{fb}^{-1}$ of integrated luminosity is a close match with the $7+8 \mathrm{TeV}$ exclusion region, with small fluctuations likely caused by different analyses and statistical fluctuations in the data.

The statistical significance expected with $3 \mathrm{ab}^{-1}$ of integrated luminosity at the LHC is shown in Fig. 4.12, A bulk of the parameter space above $M_{H}>2 m_{h}$ can be excluded at the $95 \%$ C.L., even near the decoupling limit.

\subsection{Associated $h H$ production}

Associated production of a light-heavy Higgs boson pair is a valuable complement to $H \rightarrow h$ resonant production for measuring components of the scalar potential, see 

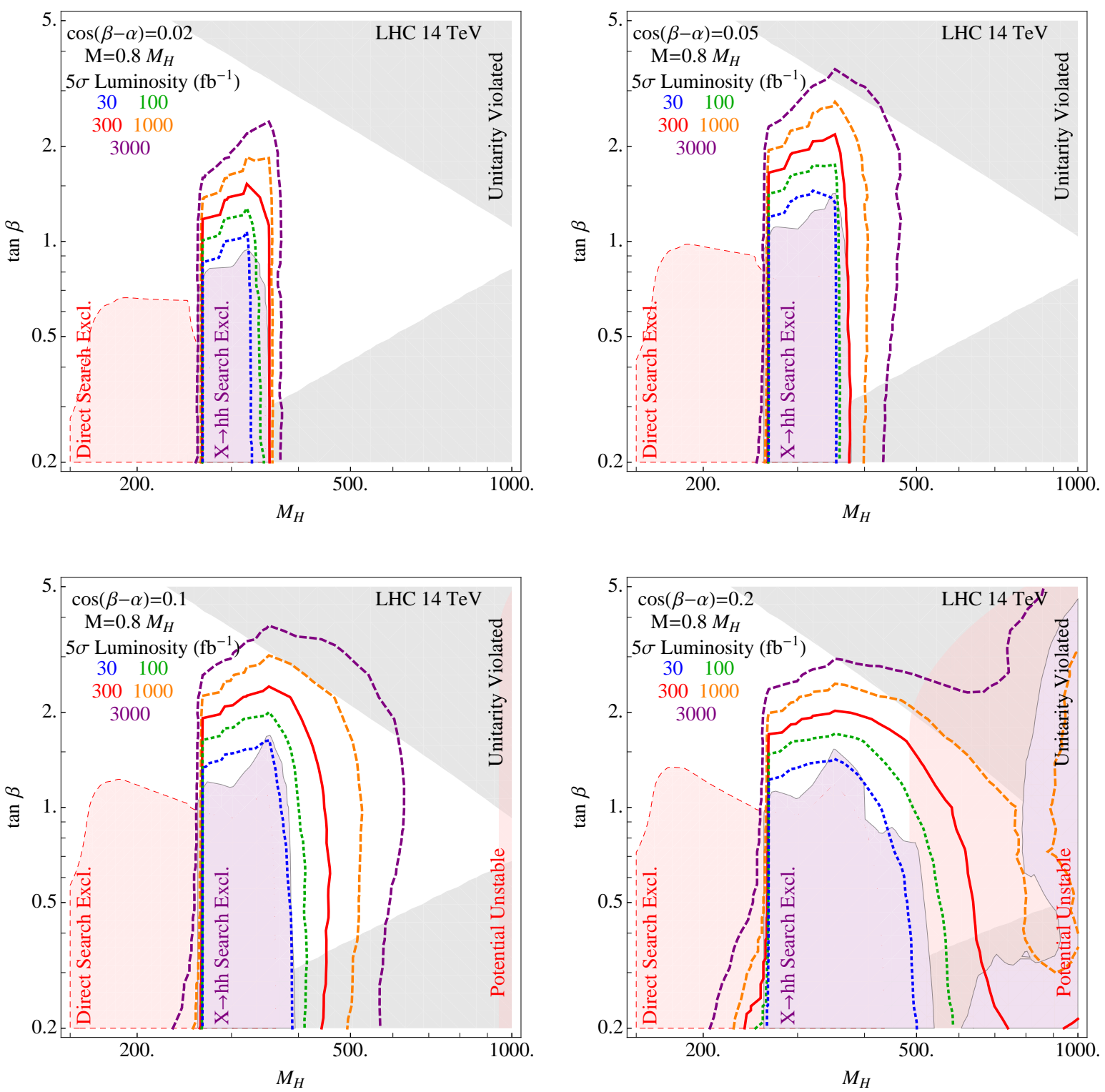

Figure 4.11 Contours of the luminosity required for $5 \sigma$ discovery in the plane of $\tan \beta$ and $M_{H}$ for selected values of $\cos (\beta-\alpha)$. Additional experimental and theoretical constraints are shown as in Fig. 4.5. 

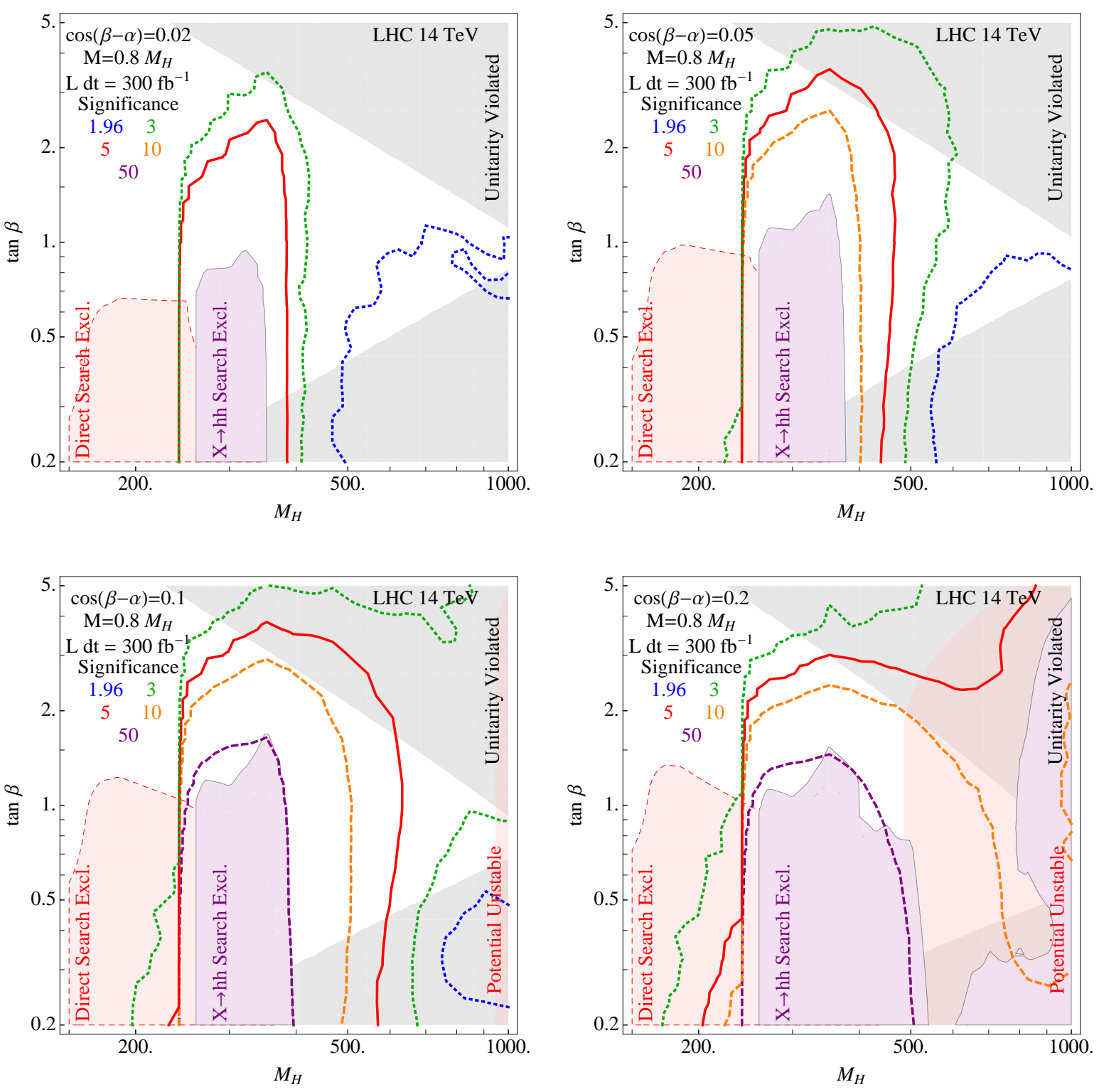

Figure 4.12 Contours of the statistical significance with $3 \mathrm{ab}^{-1}$ of integrated luminosity in the plane of $\tan \beta$ and $M_{H}$ for selected values of $\cos (\beta-\alpha)$. Additional experimental and theoretical constraints are shown as in Fig. 4.5. 
Table 4.2. This process, by virtue of the scalar coupling, $\lambda^{h H H}$, is not suppressed in the decoupling limit as seen in Eq. 4.25.

Both the box and triangle diagrams shown in Fig. 4.1 can contribute to $h H$ production. For the triangle diagram, we can have either $h$ or $H$ in the $s$-channel. Unless $M_{H}>2 m_{t}$ and $\tan \beta$ is small, the width of the heavy Higgs is narrow, so there is usually no enhancement for the $H$ diagram by being slightly off-shell. Therefore, all three diagrams are relevant.

As noted in Table 4.2, the triangle diagram involving $H$ is the only Higgs pair process that probes $\lambda^{h H H}$, as its amplitude is proportional to the combination $y_{t}^{H} \lambda^{h H H}$. Furthermore, the contribution from the $h^{*}$ triangle diagram depends on the coupling combination $y_{t}^{h} \lambda^{h h H}$, providing sensitivity to $\lambda^{h h H}$, even in regions where $B F(H \rightarrow h h)$ is small. The sensitivity is best for $\operatorname{small} \tan \beta$, due to the effect of the top Yukawa coupling to $H$ on the production cross section. More precisely, the magnitude of $y_{t}^{H}$ is largest at small $\tan \beta$, as

$$
\frac{y_{t}^{H}}{y_{t}^{h_{S M}}}=c_{\beta-\alpha}-\frac{\sqrt{1-c_{\beta-\alpha}^{2}}}{\tan \beta .}
$$

The production cross section for the $p p \rightarrow h H$ process is shown in Fig. 4.13 .

The $h H$ process can proceed to a number of final states. As above, we let the light Higgs decay to either $\gamma \gamma$ or $b \bar{b}$. The preferred final state for $H$ depends strongly on $M_{H}$, and to a lesser extent $\cos (\beta-\alpha)$ and $\tan \beta$. For $M_{H}<2 m_{h}, H$ decays predominantly into $b \bar{b}$ or $W W^{(*)} / Z Z^{(*)}$. $b \bar{b}$ is strongest for small $\cos (\beta-\alpha)$ and large $\tan \beta$, while $W W^{(*)} / Z Z^{(*)}$ is most important for large $\cos (\beta-\alpha)$, as is demonstrated by the branching fraction contours shown in Fig. 4.7 and 4.9 in Appendix 4.3, respectively. For the $H \rightarrow b \bar{b}$ channel, we find that both the $4 b$ and $b \bar{b} \gamma \gamma$ channels are viable. We also explore $H \rightarrow Z Z \rightarrow 4 \ell$ decays. We choose $Z Z \rightarrow 4 l$ despite its small branching ratio because it has small backgrounds and allows for straightforward event reconstruction. However, this limits us to choosing $h \rightarrow b \bar{b}$ in order to have a detectable number of events at the LHC. 

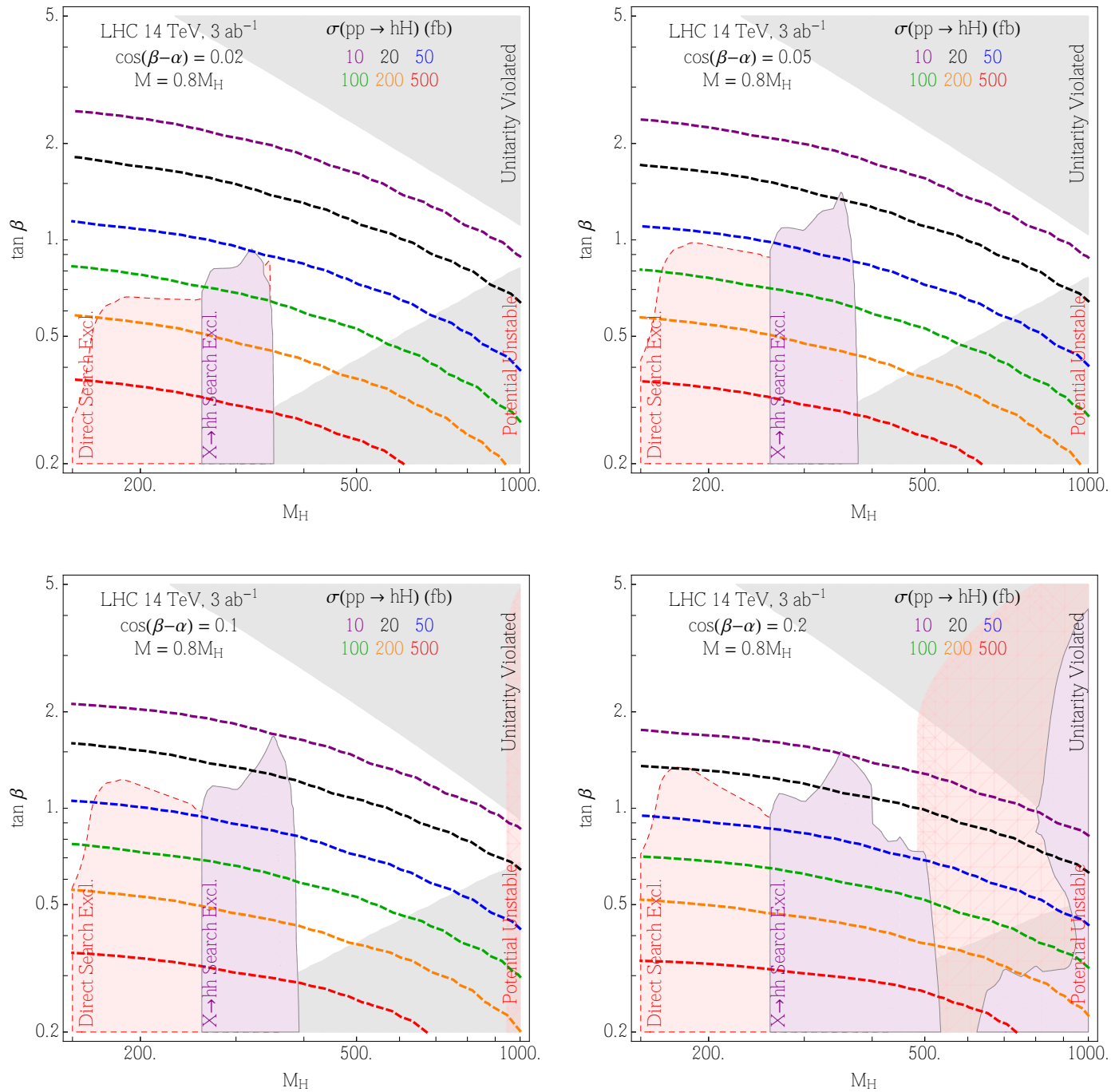

Figure 4.13 Contours of $\sigma(p p \rightarrow h H)$ in the plane of $\tan \beta$ and $M_{H}$ for selected values of $\cos (\beta-\alpha)$. Additional experimental and theoretical constraints are shown as in Fig. 4.5 . 
Above $2 m_{t}, H$ decays primarily to $t$-quarks, with a branching fraction that surpasses $90 \%$ for small $\tan \beta$ (see Fig. 4.8 in Appendix 4.3). The most viable channel in this region is $t \bar{t} b \bar{b}$, with at least one of the tops decaying leptonically to reject background. Between $2 m_{h}$ and $2 m_{t}$, the $h H \rightarrow h h h \rightarrow 4 b \gamma \gamma$ is important, as we would expect from the results of the resonant $H$ production analysis. The $H \rightarrow b \bar{b}$ and $H \rightarrow Z Z$ channels are weaker but are possibly still viable in this region as well.

We simulate the $p p \rightarrow h H$ signal using MADGRAPH as described in Section 4.5 and compute the expected LHC reach for $3 \mathrm{ab}^{-1}$ at $14 \mathrm{TeV}$. In Fig. 4.14, we show the expected $95 \%$ CL and $5 \sigma$ contours for the $b \bar{b} \gamma \gamma, 4 b, Z Z b \bar{b} 4 b \gamma \gamma, t \bar{t} b \bar{b}$ (1 lepton), and $t \bar{t} b \bar{b}$ (2 lepton) final states. As the coupling $\lambda^{h H H}$ is not suppressed in the decoupling limit, we find that our sensitivity is actually best for small $\cos (\beta-\alpha)$. Indeed, for the smallest values of $\cos (\beta-\alpha)$, we find that LHC will be able to probe essentially all of the allowed parameter space at the $95 \% \mathrm{CL}$. Even for larger values of $\cos (\beta-\alpha)$, the LHC will be sensitive to up to $\tan \beta \sim 2$ over a wide range of $M_{H}$.

In the following sections, we describe our background simulations and selection cuts for each channel. Throughout the analysis, we use the efficiencies and fake rates described in Section 4.5.1. We also include a lepton to photon fake rate of $\epsilon_{e \rightarrow \gamma}=6.2 \%$ [178]. Additionally, we apply the baseline cuts on $\Delta R$ and $p_{T}$ from the resonant $b \bar{b} \gamma \gamma$ analysis to all five channels, and add the following cuts for leptons: $\Delta R_{a b}>0.2$, $p_{T}(\ell)>20 \mathrm{GeV}$, and $\left|\eta_{\ell}\right|<2.4$.

\subsubsection{The $h H \rightarrow b \bar{b} \gamma \gamma$ channel}

The low-mass region is probed by the $H \rightarrow b \bar{b}$ channel. First let us consider the case where $h \rightarrow \gamma \gamma$. The $h \rightarrow \gamma \gamma$ branching fraction is extremely small $\left(2.3 \times 10^{-3}\right)$, but requiring photons in the final state also reduces the background significantly. The 

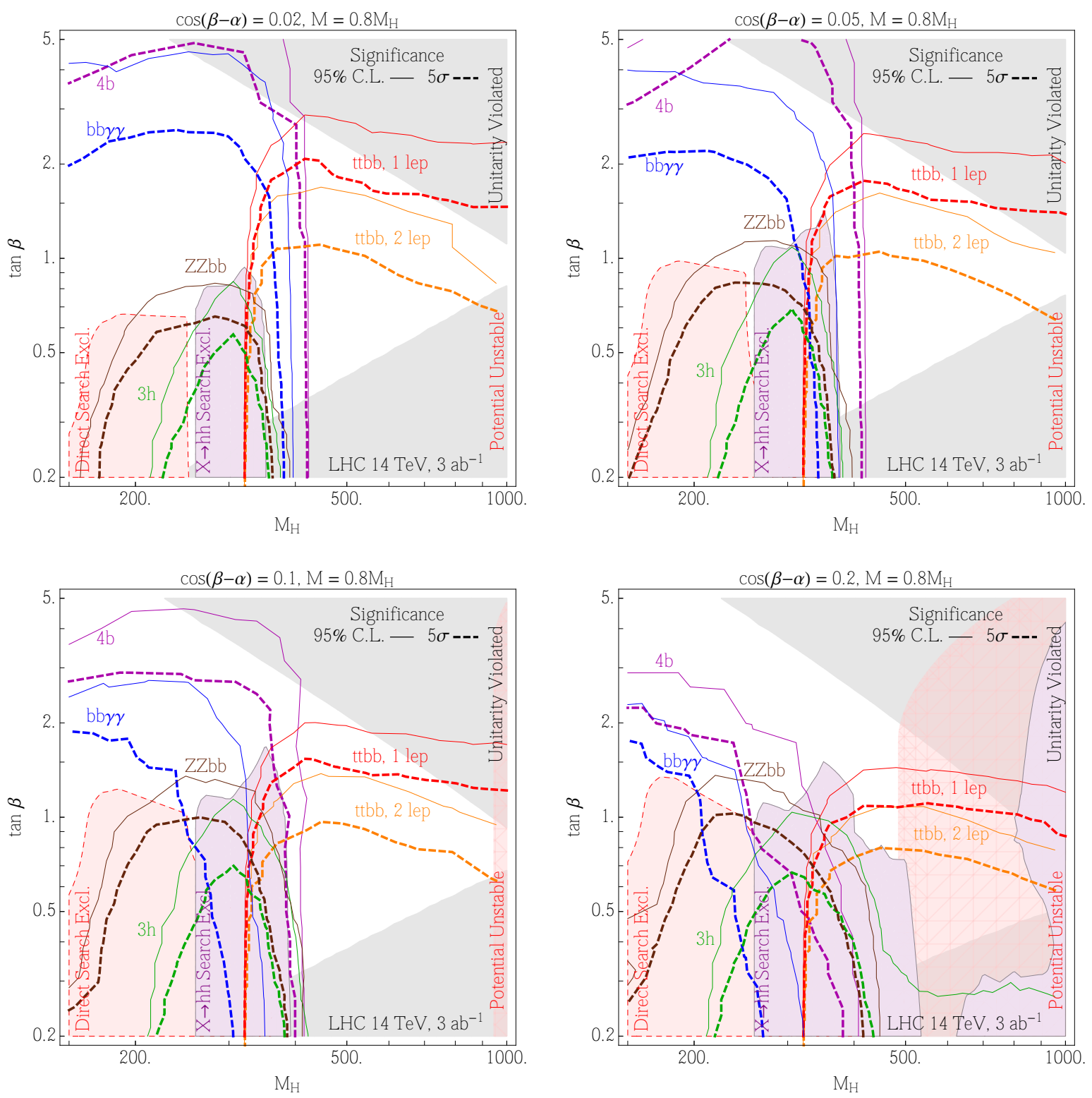

Figure 4.14 Contours of the statistical significance with $3 \mathrm{ab}^{-1}$ of integrated luminosity in the plane of $\tan \beta$ and $M_{H}$ for selected values of $\cos (\beta-\alpha)$. The bold dashed curves show the expected $5 \sigma$ significance, while the thin solid curves show the expected $95 \%$ C.L. reach. The colors correspond to different final states: $b \bar{b} \gamma \gamma$ (blue), $4 b$ (purple), $Z Z b \bar{b}$ (brown), $3 h$ (green), $t \bar{t} b \bar{b}$ in the single-lepton channel (red), and $t \bar{t} b \bar{b}$ with two leptons (orange). Additional experimental and theoretical constraints are shown as in Fig. 4.5. 
irreducible backgrounds include

$$
\begin{aligned}
& p p \rightarrow b \bar{b} \gamma \gamma \\
& p p \rightarrow b \bar{b} h \rightarrow b \bar{b} \gamma \gamma \\
& p p \rightarrow Z h \rightarrow b \bar{b} \gamma \gamma
\end{aligned}
$$

while the reducible backgrounds are

$$
\begin{aligned}
& p p \rightarrow b \bar{b} e^{+} e^{-} \quad(e \rightarrow \gamma) \\
& p p \rightarrow b \bar{b} j \gamma \quad(j \rightarrow \gamma) \\
& p p \rightarrow b \bar{b} j j \\
& p p \rightarrow j j \gamma \gamma \\
& p p \rightarrow 3 j+\gamma \\
& p p \rightarrow 4 j \text { (negligible). }
\end{aligned}
$$

We require exactly two photons and two jets, with both jets $b$-tagged. Then we apply a multivariate analysis after incorporating the following basic cuts:

$$
\left|M_{\gamma \gamma}-m_{h}\right|<5 \mathrm{GeV}, \quad M_{b \bar{b}}>100 \mathrm{GeV}
$$

The first of these cuts isolates the light Higgs resonance, while the second rejects $Z / \gamma^{*} \rightarrow$ $b \bar{b}$, as well as a significant portion of the continuum background.

From here, the procedure is exactly as it was in the resonant case discussed in Section 4.5. Specifically, we form over a discriminant

$$
\mathcal{O}=\left\{M_{b \bar{b} \gamma \gamma}, M_{b \bar{b}}, M_{\gamma \gamma}, p_{T}(b \bar{b}), p_{T}(\gamma \gamma), \Delta R_{b \bar{b}}, \Delta R_{\gamma \gamma}\right\}
$$

The expected LHC significance for $\mathcal{L}=3 \mathrm{ab}^{-1}$ is shown in blue in Fig. 4.14. The reach is extremely good for $M_{H}<400 \mathrm{GeV}$, especially for small $\cos (\beta-\alpha)$, where we achieve 95\% C.L. significance beyond $\tan \beta \approx 5$. The cross section falls of rather quickly with $\cos (\beta-\alpha)$ due to the $\cos (\beta-\alpha)$ and $\tan \beta$ dependence of the bottom Yukawa coupling 
to $H$, which is

$$
\frac{y_{b}^{H}}{y_{b}^{h_{\mathrm{SM}}}}=c_{\beta-\alpha}+\sqrt{1-c_{\beta-\alpha}^{2}} \tan \beta
$$

Hence, $y_{b}^{H}$ is enhanced for large $\tan \beta$, and this effect is strongest for small $\cos (\beta-\alpha)$.

\subsubsection{The $h H \rightarrow 4 b$ channel}

We also consider the case where $H \rightarrow b \bar{b}$, but the light Higgs decays to $b \bar{b}$ instead of $\gamma \gamma$. The signal is much larger than in the $b \bar{b} \gamma \gamma$ case, but the QCD background is large as well. With appropriate cuts, we find that the two channels are comparable in significance. The irreducible backgrounds in this case are given by

$$
\begin{aligned}
& p p \rightarrow 4 b \\
& p p \rightarrow b \bar{b} h \rightarrow 4 b .
\end{aligned}
$$

The main reducible background is $p p \rightarrow b \bar{b} j j$, with the jets faking $b$ quarks. We also considered the $4 j, t \bar{t} b \bar{b}$, and $Z h$, and $W h$ backgrounds, but found them to be negligible.

We use a cut-based analysis. We require exactly four jets, all $b$-tagged. While the $b$-tagging efficiency is low, we find that all four $b$-tags are necessary to sufficiently reduce the light jet backgrounds. Since we have more than two $b$ quarks in the final state, care must be taken in reconstructing the parent Higgs bosons. We identify the decay products of the light Higgs by minimizing $\left|M_{b_{i}, b_{j}}-m_{h}\right|$ over all possible pairs $b_{i}, b_{j}$; we label the resulting pair as $b_{1}^{h}$ and $b_{2}^{h}$. The remaining two $b$ quarks are taken to reconstruct the heavy Higgs, and are labeled $b_{1}^{H}$ and $b_{2}^{H}$. After identifying the $b$ quarks, we apply the following cuts:

$$
\begin{aligned}
M\left(b_{1}^{H} b_{2}^{H}\right) & >100 \mathrm{GeV} \\
\left|M\left(b_{1}^{h} b_{2}^{h}\right)-m_{h}\right| & <12.5 \mathrm{GeV} \\
\Delta R\left(b_{1}^{h}, b_{2}^{h}\right) & <1 \\
\Delta R\left(b_{1}^{H}, b_{2}^{H}\right) & <1.5 \\
\left|M\left(b_{1}^{H} b_{2}^{H}\right)-M_{H}\right| & <15 \mathrm{GeV} .
\end{aligned}
$$


The $\Delta R$ cuts help isolate the signal from background. The light Higgs recoils against the heavy Higgs, so the two tend to have large $p_{T}$ and be well-separated in the $\phi-\eta$ plane. Since $M_{H}>m_{h} \gg m_{b}$, the Higgs decay products tend to be cluster, especially for the light Higgs. Therefore the $\Delta R$ distributions will be peaked at small values. Furthermore, the $\Delta R$ cuts also improve the reconstruction of $M_{H}$ by ensuring the $b$ quarks have been correctly paired. In Fig. 4.15, we show the normalized $\Delta R$ distributions for both pair of $b$ quarks for $M_{H}=300 \mathrm{GeV}, \tan \beta=2$, and $\cos (\beta-\alpha)=0.1$ before cuts. In Fig. 4.16, we show the invariant mass distribution for the reconstructed heavy Higgs after the $\Delta R$ cuts for the same benchmark point.

The expected LHC significance for $\mathcal{L}=3 \mathrm{ab}^{-1}$ is shown in purple in Fig. 4.14. The $4 b$ channel is slightly stronger than the $b \bar{b} \gamma \gamma$ channel. As in the $b \bar{b} \gamma \gamma$ channel, the reach at large $\tan \beta$ is good due to the high $B F(H \rightarrow b \bar{b})$ in that region.

\subsubsection{The $h H \rightarrow Z Z b \bar{b}$ channel}

A complementary channel in the low-mass region is $H \rightarrow Z Z \rightarrow 4 \ell, h \rightarrow b \bar{b}$. The only significant background is

$$
p p \rightarrow t \bar{t} Z
$$

with the tops decaying leptonically. The potential $Z Z h, Z Z j j$ and $W W Z$ backgrounds are negligible. There are also contributions to the signal from $h \rightarrow Z Z^{*}, H \rightarrow b \bar{b}$, but they are subdominant except for a small region with $M_{H} \lesssim 200 \mathrm{GeV}, \cos (\beta-\alpha) \lesssim 0.02$ and $\tan \beta \gtrsim 5$. Furthermore, the resonant peaks in $M_{b \bar{b}}$ and $M_{4 \ell}$ are well-separated between the two cases, so there is little interference.

We require four leptons and two $b$-tags in our final state, then use the MVA to isolate the signal from the background. Our MVA variables are

$$
\mathcal{O}=\left\{M_{4 \ell}, M_{b \bar{b}}, p_{T}(b \bar{b}), \Delta R_{b \bar{b}}, \mathbb{E}_{T}\right\}
$$

The missing transverse energy variable is particularly important in this case, since $t \bar{t} Z$ has the same visible particle content as $Z Z b \bar{b}$, but with missing energy from the $W \rightarrow \ell \nu$ 


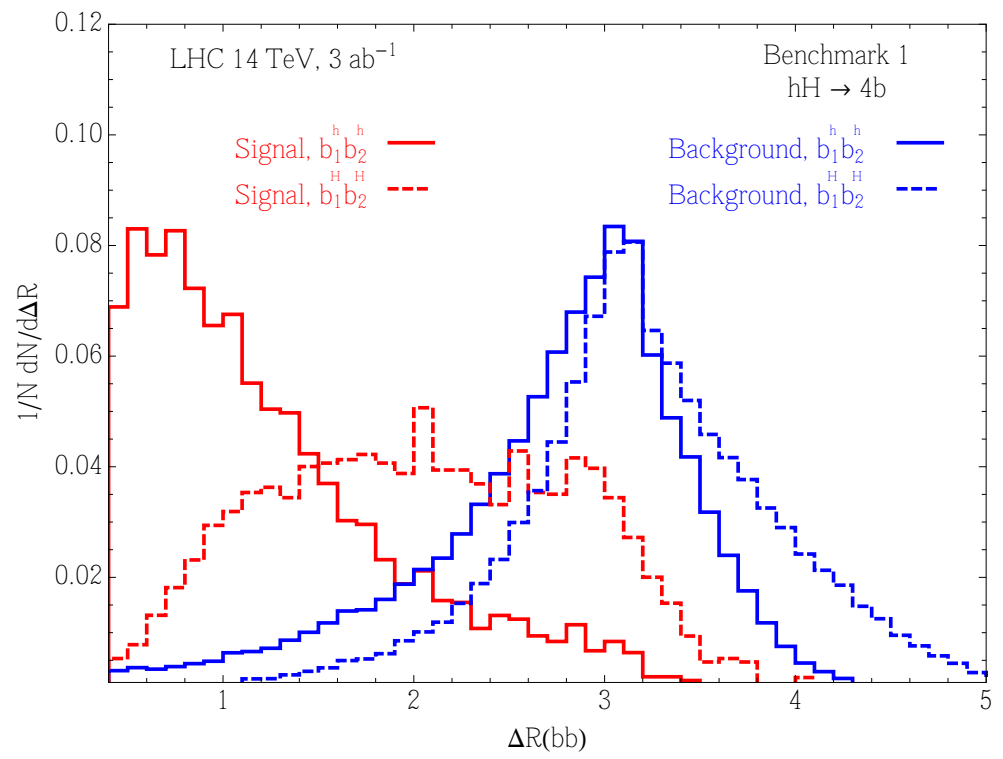

Figure 4.15 The normalized $\Delta R$ distributions for the reconstructed light Higgs $b b$ pair (solid curves) and heavy Higgs $b b$ pair (dashed curves) are shown. The signal distributions for Benchmark $1\left(M_{H}=300 \mathrm{GeV}, \tan \beta=2\right.$, and $\left.\cos (\beta-\alpha)=0.1\right)$ are shown in red, while the background is shown in blue. The signal events tend towards smaller values of $\Delta R$, indicating that the Higgs bosons (especially the $h$ ) are boosted. 


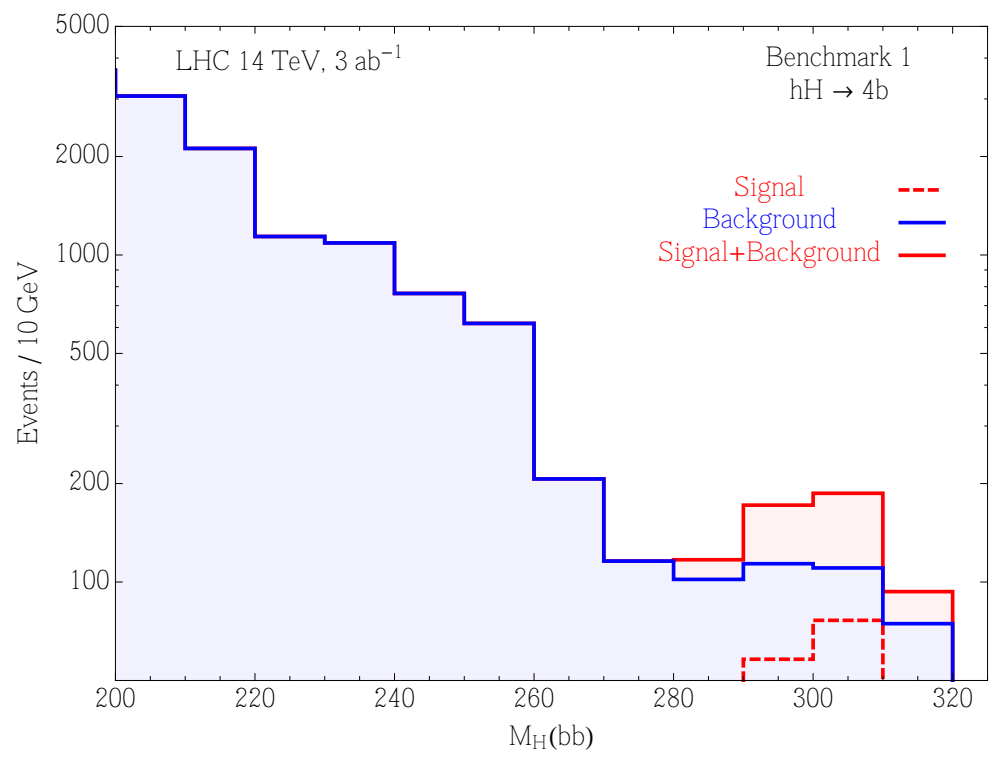

Figure 4.16 The invariant mass of the heavier $b b$ pair in the $4 b$ channel after the cuts on $M_{h}$ and $\Delta R$ is shown. Signal (red) is shown for Benchmark $1\left(M_{H}=300 \mathrm{GeV}\right.$, $\tan \beta=2$, and $\cos (\beta-\alpha)=0.1$ ). The background distribution (blue) drops off quickly with $M(b b)$, which leaves the resonance at $M_{H}$ clearly discernible. 
decays. This channel is promising for moderate values of $M_{H}$, especially for larger $\cos (\beta-\alpha)$. The LHC $3 \mathrm{ab}^{-1}$ significance is shown in brown in Fig. 4.14.

\subsubsection{The $h H \rightarrow h h h \rightarrow 4 b \gamma \gamma$ channel}

The $4 b \gamma \gamma$ backgrounds are fairly small. The relevant backgrounds are

$$
\begin{aligned}
& p p \rightarrow 4 b \gamma \gamma \\
& p p \rightarrow b \bar{b} j j \gamma \gamma
\end{aligned}
$$

Backgrounds with higher light jet multiplicities are negligible due to the small $j \rightarrow \gamma$ and $j \rightarrow b$ fake rates.

Our initial selection requires exactly four $b$-tagged jets and two photons. The diphoton invariant mass must satisfy

$$
\left|M_{\gamma \gamma}-m_{h}\right|<5 \mathrm{GeV}
$$

This cut is sufficient to optimize the cut-based significance, reducing the background to only $\sim 2$ events for $3 \mathrm{ab}^{-1}$. However, we can better reconstruct the heavy Higgs mass with an additional cut. First we pair the $b$ quarks by minimizing $\left|M_{b_{i}, b_{j}}-M_{h}\right|$ over all possible pairs $b_{i}, b_{j}$, as in the $4 b$ final state. We denote the three reconstructed light Higgs bosons as $\left(h_{\gamma}, h_{b 1}, h_{b 2}\right)$, where $\left|M\left(h_{b 1}\right)-M_{h}\right|<\left|M\left(h_{b 2}\right)-M_{h}\right|$. If we compute $\Delta R\left(h_{i}, h_{j}\right)$ for each of the reconstructed light Higgs bosons, we find that the distribution is peaked at low $\Delta R$ and near $\Delta R \approx \pi$. This corresponds to two light Higgs bosons from the $H$ decay being clustered together and the other $h$ recoiling against the $H \rightarrow h h$ system. We therefore require that exactly one pair $\left(h_{i}, h_{j}\right)$ satisfy

$$
\Delta R\left(h_{i}, h_{j}\right)<1.5
$$

and use that pair to reconstruct the heavy Higgs.

The expected LHC significance for $\mathcal{L}=3 \mathrm{ab}^{-1}$ is shown in green in Fig. 4.14. Unsurprisingly, the significance contours run parallel those found for the $H \rightarrow h$ h resonant case. 


\subsubsection{The $h H \rightarrow b \bar{b} t \bar{t}$ channel}

To explore the $M_{H} \gtrsim 2 m_{t}$ region, we consider the $b \bar{b} t \bar{t}$ final state. The irreducible backgrounds include:

$$
\begin{aligned}
& p p \rightarrow b \bar{b} t \bar{t} \\
& p p \rightarrow h t \bar{t} \rightarrow b \bar{b} t \bar{t} \\
& p p \rightarrow Z t \bar{t} \rightarrow b \bar{b} t \bar{t} .
\end{aligned}
$$

We also include the reducible background $p p \rightarrow j j t \bar{t}$.

We require four $b$-tags in our final state. At least one of the top quarks must decay via $t \rightarrow b W \rightarrow b \ell \nu$. We allow the other top to decay to $b j j$ or $b \ell \nu$. Thus our final state must include either $4 j+2 \ell+\mathbb{E}_{T}$ or $6 j+\ell+\mathbb{E}_{T}$. As above, we reconstruct the light Higgs by minimizing $\left|M_{b_{i}, b_{j}}-m_{h}\right|$ and requiring that this pair of $b$ quarks satisfies

$$
\left|M\left(b_{1}^{h} b_{2}^{h}\right)-m_{h}\right|<12.5 \mathrm{GeV}
$$

and

$$
\Delta R\left(b_{1}^{h} b_{2}^{h}\right)<1.0
$$

The other two $b$ quarks are assumed to come from top decays. The $\Delta R\left(b_{1}^{h} b_{2}^{h}\right)$ is very peaked in this channel, since $H$ must be heavy to allow for $t \bar{t}$ decays. This leads to a more boosted light Higgs than in the previous channels, and therefore more closely clustered $b$ quarks.

In the one-lepton channel, we apply additional cuts. We can reconstruct the tops by minimizing $\left|M\left(b_{i} j j\right)-m_{t}\right|$ over the remaining two $b$ quarks. Let $M\left(t_{h}\right)=M(b j j)$ and $M_{T}\left(t_{l}\right)=M_{T}\left(b l \mathbb{E}_{T}\right)$. Then we require

$$
\left|M\left(t_{h}\right)-m_{t}\right|<20 \mathrm{GeV}
$$

and

$$
M_{T}\left(t_{l}\right)<m_{t}
$$




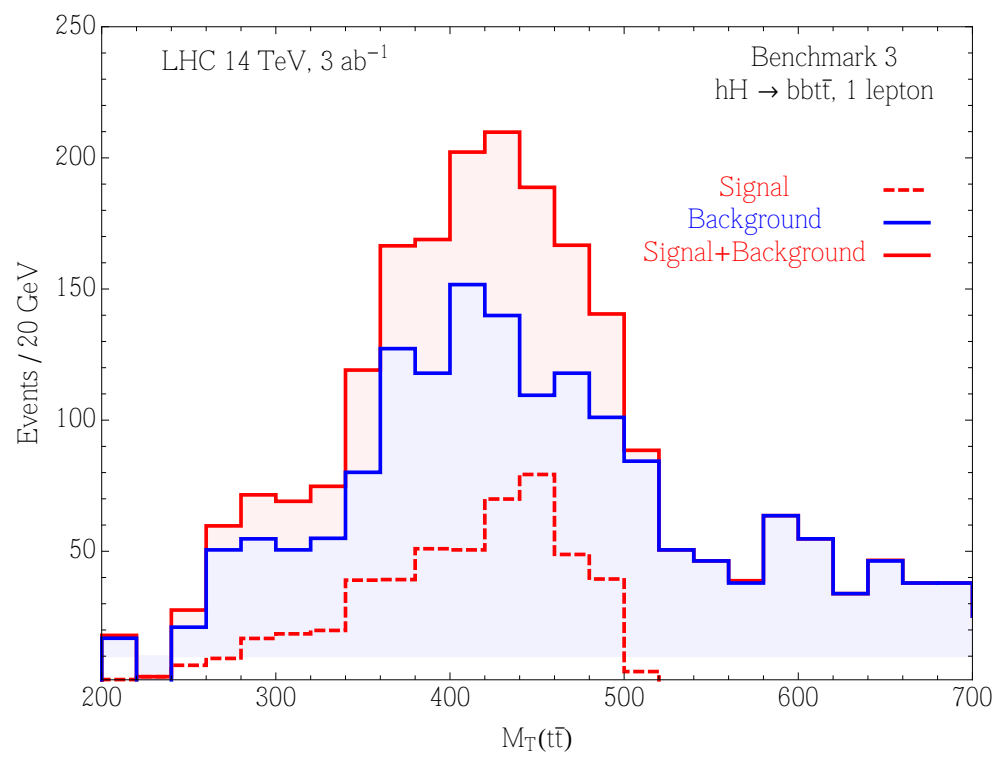

Figure 4.17 The transverse mass of the top quark pair in the $t \bar{t} b \bar{b}$ with one lepton channel. The signal (red) is shown for Benchmark $3(\cos (\beta-\alpha)=0.02, \tan \beta=1$, and $M_{H}=500 \mathrm{GeV}$ ). The signal drops off sharply above $M_{H}$, while the background (blue) decreases more gradually.

Finally, we define a signal region that varies with $M_{H}$ :

$$
M_{H}-200 \mathrm{GeV}<M_{T}\left(t_{h} t_{l}\right)<M_{H}-10 \mathrm{GeV} .
$$

In Fig. 4.17, we show the transverse mass of the top quark pair after cuts for $\cos (\beta-\alpha)=$ $0.02, \tan \beta=1$, and $M_{H}=500 \mathrm{GeV}$. The one-lepton channel is stronger than the twolepton channel due to the relatively small branching fraction for $W \rightarrow \ell \nu$. The expected LHC significances for $\mathcal{L}=3 \mathrm{ab}^{-1}$ are shown in red and orange in Fig. 4.14. The reach decreases slowly with $M_{H}$, and it should be possible to probe above $M_{H}=1 \mathrm{TeV}$ for $\tan \beta<2$ at the LHC.

\subsection{Summary of 2HDM Analysis}

We have investigated two types of Higgs pair production within the CP-conserving Type-II 2HDM: the resonant production of an $h h$ pair, and the associated production of an $h H$ pair. We included theoretical constraints from requiring perturbative unitarity 
and a bounded scalar potential, as well as LHC constraints from the direct heavy Higgs search and the $X \rightarrow h$ search. We have made the simplifying assumptions that $M_{H}=$ $M_{H \pm}=M_{A}$, and $M=0.8 M_{H}$ and have presented our results in terms of the remaining free parameters: $M_{H}, \tan \beta$, and $\cos (\beta-\alpha)$.

For the resonant case of $p p \rightarrow H \rightarrow h h$, the reach in the $b \bar{b} \gamma \gamma$ channel for $30 \mathrm{fb}^{-1}$ at LHC14 is comparable to the current limits on $X \rightarrow h h$, as expected. With $3 \mathrm{ab}^{-1}$, the coverage extends to $\tan \beta \approx 2$ and $M_{H} \approx 350 \mathrm{GeV}$ near the decoupling limit. For large $\tan \beta$, the reach improves so that a majority of the theoretically allowed region above $M_{H}=2 m_{h}$ may be probed. This is because the $H \rightarrow h h$ rate is governed by the $\lambda^{h h H}$ coupling, which behaves as $\cos (\beta-\alpha)$ to leading order and is suppressed in the decoupling limit.

The associated production case, $p p \rightarrow h H$, offers a variety of interesting channels to explore. Near the decoupling limit, the LHC14 reach is excellent due to the nondecoupling nature of the $\lambda^{h h H}$ scalar coupling. Due to the potentially large mass difference between light and heavy Higgs states, the $h$ is often boosted when $M_{H} \gg m_{h}$, resulting in decay products which have small separation. This is contrary to the common backgrounds, which contain more dispersed jets and leptons, resulting in a quite clean differentiation between signal from background. In the low mass region, $M_{H}<2 m_{h} \simeq 250$ $\mathrm{GeV}$, the $H \rightarrow b b, h \rightarrow b \bar{b} / \gamma \gamma$ channels cover the entire allowed range of $\tan \beta$. The $H h \rightarrow t \bar{t} b \bar{b}$ channels cover the high mass region, $M_{H}>2 m_{t} \simeq 350 \mathrm{GeV}$. For larger values of $\cos (\beta-\alpha)$, the sensitivity in these channels decreases due to the increased $B F(H \rightarrow W W / Z Z)$, when kinematically allowed. However, $H \rightarrow Z Z$ and $H \rightarrow h h$ improve the reach in this region.

In our analysis, we selected three benchmark points that illustrate the discovery potential for different channels, which were presented in Table 4.1. Point A, for which $M_{H}=300 \mathrm{GeV}, \tan \beta=2$, and $\cos (\beta-\alpha)=0.1$, demonstrated the viability of the $H \rightarrow h h \rightarrow b \bar{b} \gamma \gamma$ channel due to the large $\mathrm{BF}(H \rightarrow h h)$. A secondary channel that is viable is the $H h \rightarrow b \bar{b} b \bar{b}$ mode. Point $\mathrm{B}$, for which $M_{H}=300 \mathrm{GeV}, \tan \beta=1$, 
and $\cos (\beta-\alpha)=0.02$, highlighted the $h h / h H \rightarrow b \bar{b} \gamma \gamma$ and $b \bar{b} b \bar{b}$ channels. The large $\mathrm{BF}(H \rightarrow b \bar{b})$ provides a sizable rate to the $b \bar{b} \gamma \gamma$ and $b \bar{b} b \bar{b}$ final states. The $h h \rightarrow b \bar{b} \gamma \gamma$ channel has high significance due to the large production cross section of $p p \rightarrow h h$. Point $\mathrm{C}$, for which $M_{H}=500 \mathrm{GeV}, \tan \beta=1$, and $\cos (\beta-\alpha)=0.02$, highlighted the $h H \rightarrow t \bar{t} b \bar{b}$ channel. In this case, $\mathrm{BF}(H \rightarrow t \bar{t})$ is large, allowing a sizable rate for the final state. We present the statistical significance for these points at LHC14 with $3 \mathrm{ab}^{-1}$ of integrated luminosity in Table 4.3 .

Ultimately, the results of our analysis demonstrate that there is a large region of the CP-conserving Type-II 2HDM parameter space that is currently unconstrained, but should be testable by the LHC $14 \mathrm{TeV}$ run. Resonant production of $h$ h pairs and associated production of $h H$ pairs are orthogonal probes of the 2HDM scalar potential. By considering both production modes, along with the continuum production of $h h$ pairs, the LHC should be able to measure the three triscalar couplings $\left(\lambda^{h h h}, \lambda^{h h H}\right.$, $\left.\lambda^{h H H}\right)$. These coupling measurements can then be checked for consistency with a given model in order to illuminate the structure of the underlying scalar sector. 


\begin{tabular}{|c|c|c|c|}
\cline { 2 - 4 } & $\begin{array}{c}\text { A: } \\
M_{H}=300 \mathrm{GeV},\end{array}$ & $\begin{array}{c}\text { B: } \\
M_{H}=300 \mathrm{GeV},\end{array}$ & $\begin{array}{c}\text { C: } \\
M_{H}=500 \mathrm{GeV}, \\
t_{\beta}=2, c_{\beta-\alpha}=0.1\end{array}$ \\
$t_{\beta}=1, c_{\beta-\alpha}=0.02$ & $t_{\beta}=1, c_{\beta-\alpha}=0.02$ \\
\hline$\lambda^{h h h} / \lambda_{S M}^{h h h}$ & 0.946 & 0.998 & 0.992 \\
$\lambda^{h h H}(\mathrm{GeV})$ & 40.8 & 8.87 & 29.2 \\
$\lambda^{h H H}(\mathrm{GeV})$ & 310 & 327 & 795 \\
$y_{t}^{H}$ & -0.40 & -0.98 & -0.98 \\
\hline$\sigma(p p \rightarrow h h)(\mathrm{fb})$ & 340 & 810 & 37 \\
$\sigma(p p \rightarrow h H)(\mathrm{fb})$ & 7.7 & 44 & 26 \\
\hline$B F(H \rightarrow h h)$ & $18 \%$ & $7.6 \%$ & $0.1 \%$ \\
$B F(H \rightarrow t t)$ & $0.0 \%$ & $0.0 \%$ & $0.2 \%$ \\
$B F(H \rightarrow b b)$ & $34 \%$ & $74 \%$ & $0.2 \%$ \\
\hline$B F(H \rightarrow Z Z+W W)$ & $49 \%$ & $18 \%$ & \\
\hline
\end{tabular}

Table 4.1 Benchmark points of relevant couplings, production cross sections at LHC14 and branching fractions for the channels of interest.

\begin{tabular}{|c|ccc|}
\hline Process & $\lambda^{h h h}$ & $\lambda^{h h H}$ & $\lambda^{h H H}$ \\
\hline$p p \rightarrow h h$ (continuum) & $\checkmark$ & $\times$ & $\times$ \\
$p p \rightarrow H \rightarrow h h$ & $\checkmark$ & $\checkmark$ & $\times$ \\
$p p \rightarrow h^{*} / H^{*} \rightarrow h H$ & $\checkmark$ & $\checkmark$ & $\checkmark$ \\
\hline Decoupling dependence & $\lambda_{\mathrm{SM}}^{h h h}\left(1+\mathcal{O}\left(c_{\beta-\alpha}^{2}\right)\right)$ & $\mathcal{O}\left(c_{\beta-\alpha}\right)$ & $\left(2 M_{H}^{2}-2 M^{2}+M_{h}^{2}\right) / v+\mathcal{O}\left(c_{\beta-\alpha}\right)$ \\
\hline
\end{tabular}

Table 4.2 The Higgs pair production processes that are sensitive to the couplings among the CP-even states. For each scalar coupling, the leading term in the expansion in the decoupling parameter, $c_{\beta-\alpha}=\cos (\beta-\alpha)$, is also shown. 


\begin{tabular}{|c|c|c|c|}
\hline & $\begin{array}{c}\mathbf{A}: \\
M_{H}=300 \mathrm{GeV} \\
t_{\beta}=2, c_{\beta-\alpha}=0.1\end{array}$ & $\begin{array}{c}\text { B: } \\
M_{H}=300 \mathrm{GeV} \\
t_{\beta}=1, c_{\beta-\alpha}=0.02\end{array}$ & $\begin{array}{c}\mathrm{C}: \\
M_{H}=500 \mathrm{GeV} \\
t_{\beta}=1, c_{\beta-\alpha}=0.02\end{array}$ \\
\hline$\lambda^{h h h} / \lambda_{S M}^{h h h}$ & 0.946 & 0.998 & 0.992 \\
\hline$\lambda^{h h H}(\mathrm{GeV})$ & 40.8 & 8.87 & 29.2 \\
\hline$\lambda^{h H H}(\mathrm{GeV})$ & 310 & 327 & 795 \\
\hline$y_{t}^{H}$ & -0.40 & -0.98 & -0.98 \\
\hline$\sigma(p p \rightarrow h h)(\mathrm{fb})$ & 340 & 810 & 37 \\
\hline$\sigma(p p \rightarrow h H)(\mathrm{fb})$ & 7.7 & 44 & 26 \\
\hline$B F(H \rightarrow h h)$ & $18 \%$ & $7.6 \%$ & $0.1 \%$ \\
\hline$B F(H \rightarrow t t)$ & $0.0 \%$ & $0.0 \%$ & $99 \%$ \\
\hline$B F(H \rightarrow b b)$ & $34 \%$ & $74 \%$ & $0.2 \%$ \\
\hline$B F(H \rightarrow Z Z+W W)$ & $49 \%$ & $18 \%$ & $0.2 \%$ \\
\hline $\mathcal{S}(H \rightarrow h h)$ & 22 & 55 & 2.4 \\
\hline $\mathcal{S}(H h \rightarrow 3 h \rightarrow b \bar{b} b \bar{b} \gamma \gamma)$ & 0.38 & 1.2 & 0.0 \\
\hline $\mathcal{S}(H h \rightarrow b \bar{b} \gamma \gamma)$ & 2.5 & 14 & 0.0 \\
\hline $\mathcal{S}(H h \rightarrow b \bar{b} b \bar{b})$ & 8.2 & 68 & 0.0 \\
\hline $\mathcal{S}\left(H h \rightarrow t_{h} t_{\ell} b \bar{b}\right)$ & 0.0 & 0.0 & 16 \\
\hline $\mathcal{S}\left(H h \rightarrow t_{\ell} t_{\ell} b \bar{b}\right)$ & 0.0 & 0.0 & 5.6 \\
\hline $\mathcal{S}(H h \rightarrow Z Z b \bar{b})$ & 0.62 & 0.48 & 0.0 \\
\hline
\end{tabular}

Table 4.3 The three benchmark points chosen to help elucidate the most viable channels as in Table 4.1, but with the expected statistical significance for LHC14 with $3 \mathrm{ab}^{-1}$ of integrated luminosity. 


\section{LIST OF REFERENCES}

[1] CMS Collaboration, "Precise determination of the mass of the Higgs boson," CMSPAS-HIG-14-009, 2014.

[2] J. Beringer et al. [Particle Data Group Collaboration], "Review of Particle Physics (RPP)," Phys. Rev. D 86, 010001 (2012).

[3] SHeinemeyer et al. [LHC Higgs Cross Section Working Group Collaboration], arXiv:1307.1347 [hep-ph].

[4] LHC Higgs Cross Section Working Group, https://twiki.cern.ch/twiki/bin/view/LHCPhysic

[5] P. Langacker, "The Physics of Heavy $Z^{\prime}$ Gauge Bosons," Rev. Mod. Phys. 81 (2009) 1199-1228, arXiv:0801.1345 [hep-ph].

[6] M. S. Carena, A. Daleo, B. A. Dobrescu, and T. M. P. Tait, " $Z$ ' gauge bosons at the Tevatron," Phys. Rev. D70 (2004) 093009, arXiv:hep-ph/0408098.

[7] A. Leike, "The Phenomenology of extra neutral gauge bosons," Phys. Rept. 317 (1999) 143-250, arXiv:hep-ph/9805494.

[8] J. L. Hewett and T. G. Rizzo, "Low-Energy Phenomenology of Superstring Inspired E(6) Models," Phys. Rept. 183 (1989) 193.

[9] D. London and J. L. Rosner, "Extra Gauge Bosons in E(6)," Phys.Rev. D34 (1986) 1530 .

[10] M. T. Frandsen, F. Kahlhoefer, S. Sarkar, and K. Schmidt-Hoberg, "Direct detection of dark matter in models with a light $Z^{\prime}$," JHEP 09 (2011) 128, arXiv:1107.2118 [hep-ph].

[11] J. L. Feng, J. Kumar, D. Marfatia, and D. Sanford, "Isospin-Violating Dark Matter," Phys.Lett. B703 (2011) 124-127, arXiv:1102.4331 [hep-ph].

[12] R. Essig, J. A. Jaros, W. Wester, P. H. Adrian, S. Andreas, T. Averett, O. Baker and B. Batell et al., arXiv:1311.0029 [hep-ph]. 
[13] See e.g, R. N. Mohapatra, "Unification and supersymmetry. The frontiers of quark - lepton physics," New York, USA: Springer (2003) 421 p;

[14] P. Langacker and S. U. Sankar, "Bounds on the mass of $W_{R}$ and the $W_{L}-W_{R}$ mixing angle $\xi$ in general $S U(2)_{L} \times S U(2)_{R} \times U(1)$ models," Phys. Rev. D 40, 1569 (1989);

[15] V. D. Barger, K. Whisnant and W.-Y. Keung, "Gauge model with small $C_{q}^{2}$ interaction term and heavy weak bosons," Phys. Rev. D 25, 291 (1982);

[16] K. Hsieh, K. Schmitz, J.-H. Yu and C.-P. Yuan, "Global analysis of general $S U(2) \times S U(2) \times U(1)$ models with precision data", Phys. Rev. D 82, 035011 (2010) [arXiv:1003.3482];

[17] T. Abe, N. Chen and H. -J. He, "LHC Higgs signatures from extended electroweak gauge symmetry", JHEP 1301, 082 (2013) [arXiv:1207.4103].

[18] G. C. Branco, P. M. Ferreira, L. Lavoura, M. N. Rebelo, M. Sher and J. P. Silva, Phys. Rept. 516, 1 (2012) [arXiv:1106.0034 [hep-ph]].

[19] CMS Collaboration [CMS Collaboration], "Search for Resonances in the Dilepton Mass Distribution in pp Collisions at sqrt(s) $=8 \mathrm{TeV}$," CMS-PAS-EXO-12-061.

[20] CMS Collaboration, "Search for leptonic decays of $W^{\prime}$ bosons in $p p$ collisions at $\sqrt{s}=8 \mathrm{TeV}$ ", report CMS-PAS-EXO-12-060, March 2013.

[21] S. Chatrchyan et al. [CMS Collaboration], Eur. Phys. J. C 73, 2469 (2013) [arXiv:1304.0213 [hep-ex]].

[22] R. Gavin, Y. Li, F. Petriello, and S. Quackenbush, "W physics at the LHC with FEWZ 2.1," arXiv:1201.5896 [hep-ph].

[23] R. Gavin, Y. Li, F. Petriello, and S. Quackenbush, "FEWZ 2.0: A code for hadronic $Z$ production at next-to- next-to-leading order," Comput. Phys. Commun. 182 (2011) 2388-2403, arXiv:1011.3540 [hep-ph].

[24] CMS Collaboration, S. Chatrchyan et al., "Search for narrow resonances in dilepton mass spectra in pp collisions at sqrt(s) $=7 \mathrm{TeV}, "$ arXiv:1206.1849 [hep-ex].

[25] ATLAS Collaboration, G. Aad et al., "Search for dilepton resonances in pp collisions at $\sqrt{(s)}=7 \mathrm{TeV}$ with the ATLAS detector," Phys.Rev.Lett. 107 (2011) 272002, arXiv:1108.1582 [hep-ex].

[26] CMS Collaboration, V. Timciuc, "Search for High-Mass Resonances in the Dilepton Final State with the CMS Detector," arXiv:1111.4528 [hep-ex]. 
[27] XENON100 Collaboration, E. Aprile et al., "Dark Matter Results from 225 Live Days of XENON100 Data," Phys.Rev.Lett. 109 (2012) 181301, arXiv:1207.5988 [astro-ph.CO].

[28] J. Erler, P. Langacker, S. Munir, and E. Rojas, " $Z$ ' Bosons at Colliders: a Bayesian Viewpoint," arXiv:1103.2659 [hep-ph].

[29] J. Erler, P. Langacker, S. Munir, and E. Rojas, " $Z^{\prime}$ Bosons from E(6): Collider and Electroweak Constraints," arXiv:1108.0685 [hep-ph].

[30] M. Cvetic and S. Godfrey, "Discovery and identification of extra gauge bosons," arXiv:hep-ph/9504216 [hep-ph]. Summary of the Working Subgroup on Extra Gauge Bosons of the DPF long-range planning study to be published in Electroweak Symmetry Breaking and Beyond the Standard Model, eds. T. Barklow, S. Dawson, H. Haber and J. Siegrist (World Scientific 1995).

[31] T. G. Rizzo, " $Z^{\prime}$ phenomenology and the LHC," arXiv:hep-ph/0610104 [hep-ph].

[32] F. Petriello and S. Quackenbush, "Measuring $Z$ ' couplings at the CERN LHC," Phys. Rev. D77 (2008) 115004, arXiv:0801.4389 [hep-ph].

[33] M. Dittmar, A.-S. Nicollerat, and A. Djouadi, " $Z$ ' studies at the LHC: An update," Phys. Lett. B583 (2004) 111-120, arXiv:hep-ph/0307020.

[34] CMS Collaboration, S. Chatrchyan et al., "Search for high mass resonances decaying into tau-lepton pairs in pp collisions at sqrt(s) $=7 \mathrm{TeV}$," arXiv:1206.1725 [hep-ex].

[35] E. Accomando, A. Belyaev, L. Fedeli, S. F. King, and C. ShepherdThemistocleous, "Z' physics with early LHC data," Phys.Rev. D83 (2011) 075012, arXiv:1010.6058 [hep-ph].

[36] C.-F. Chang, K. Cheung, and T.-C. Yuan, "Supersymmetric Decays of the Z' Boson," JHEP 1109 (2011) 058, arXiv:1107.1133 [hep-ph].

[37] V. Barger, T. Han, and D. G. Walker, "Top Quark Pairs at High Invariant Mass: A Model-Independent Discriminator of New Physics at the LHC," Phys.Rev.Lett. 100 (2008) 031801, arXiv:hep-ph/0612016 [hep-ph].

[38] F. del Aguila, J. Aguilar-Saavedra, M. Moretti, F. Piccinini, R. Pittau, et al., "Combined analysis of $Z^{\prime} \rightarrow t \bar{t}$ and $Z^{\prime} \rightarrow t \bar{t} j$ production for vector resonance searches at LHC," Phys.Lett. B685 (2010) 302-308, arXiv:0912.3799 [hep-ph].

[39] Do Collaboration, V. M. Abazov et al., "Search for a Narrow $t \bar{t}$ Resonance in $p \bar{p}$ Collisions at $\sqrt{s}=1.96 \mathrm{TeV}$," arXiv:1111.1271 [hep-ex]. 
[40] C.-W. Chiang, N. D. Christensen, G.-J. Ding, and T. Han, "Discovery in DrellYan Processes at the LHC," Phys.Rev. D85 (2012) 015023, arXiv:1107.5830 [hep-ph].

[41] S. Godfrey and T. A. Martin, "Identification of Extra Neutral Gauge Bosons at the LHC Using b- and t-Quarks," Phys.Rev.Lett. 101 (2008) 151803, arXiv:0807.1080 [hep-ph].

[42] R. Diener, S. Godfrey, and T. A. Martin, "Discovery and Identification of Extra Neutral Gauge Bosons at the LHC," arXiv:0910.1334 [hep-ph].

[43] R. Diener, S. Godfrey, and T. A. Martin, "Unravelling an Extra Neutral Gauge Boson at the LHC using Third Generation Fermions," Phys.Rev. D83 (2011) 115008, arXiv:1006.2845 [hep-ph].

[44] O. Eboli, J. Gonzalez-Fraile, and M. Gonzalez-Garcia, "Present Bounds on New Neutral Vector Resonances from Electroweak Gauge Boson Pair Production at the LHC," arXiv:1112.0316 [hep-ph].

[45] R. Diener, S. Godfrey, and I. Turan, "Constraining Extra Neutral Gauge Bosons with Atomic Parity Violation Measurements," arXiv:1111.4566 [hep-ph].

[46] V. D. Barger and K. Whisnant, "Heavy Z Boson Decays to Two Bosons in E(6) Superstring Models," Phys.Rev. D36 (1987) 3429.

[47] V. D. Barger, K. Cheung, and P. Langacker, "Baryonic Z-prime connection of LEP R(b,c) data with Tevatron (W, Z, gamma) b anti-b events," Phys.Lett. B381 (1996) 226-236, arXiv:hep-ph/9604298 [hep-ph].

[48] P. Osland, A. Pankov, A. Tsytrinov, and N. Paver, "Spin and model identification of $Z^{\prime}$ bosons at the LHC," Phys.Rev. D79 (2009) 115021, arXiv:0904.4857 [hep-ph].

[49] A. Martin, W. Stirling, R. Thorne, and G. Watt, "Parton distributions for the LHC," Eur.Phys.J. C63 (2009) 189-285, arXiv:0901.0002 [hep-ph].

[50] V. Barger, P. Fileviez Perez, and S. Spinner, "Minimal gauged U(1)(B-L) model with spontaneous R-parity violation," Phys.Rev.Lett. 102 (2009) 181802, arXiv:0812.3661 [hep-ph].

[51] P. Fileviez Perez and S. Spinner, "The Minimal Theory for R-parity Violation at the LHC," arXiv:1201.5923 [hep-ph].

[52] V. Braun, Y.-H. He, B. A. Ovrut, and T. Pantev, "A Heterotic standard model," Phys.Lett. B618 (2005) 252-258, arXiv:hep-th/0501070 [hep-th]. 
[53] B. A. Ovrut, A. Purves, and S. Spinner, "Wilson Lines and a Canonical Basis of SU(4) Heterotic Standard Models," arXiv:1203.1325 [hep-th].

[54] L. A. Anchordoqui, I. Antoniadis, H. Goldberg, X. Huang, D. Lust, et al., "LHC Phenomenology and Cosmology of Intersecting D-Brane Models," arXiv:1206.2537 [hep-ph].

[55] V. D. Barger, N. Deshpande, and K. Whisnant, "Phenomenology of $E(6)$ Electroweak Models with Two Extra $Z$ Bosons," Phys.Rev. D35 (1987) 1005.

[56] R. Mohapatra, Unification and Supersymmetry: The Frontiers of Quark-Lepton Physics. Graduate Texts in Contemporary Physics. Springer-Verlag, 2003. http: //books.google.com/books?id=ai12StX-DNoC.

[57] Particle Data Group Collaboration, K. Nakamura et al., "Review of particle physics," J. Phys. G37 (2010) 075021.

[58] J. C. Collins and D. E. Soper, "Angular distribution of dileptons in high-energy hadron collisions," Phys. Rev. D 16 (Oct, 1977) 2219-2225, http://link.aps . org/doi/10.1103/PhysRevD.16.2219.

[59] A. de Gouvea, A. Friedland, and H. Murayama, "Seasonal variations of the Be7 solar neutrino flux," Phys.Rev. D60 (1999) 093011, arXiv:hep-ph/9904399 [hep-ph].

[60] H. An, X. Ji, and L.-T. Wang, "Light dark matter and $Z^{\prime}$ dark force at colliders," arXiv:1202.2894 [hep-ph].

[61] M. R. Buckley, D. Hooper, and J. L. Rosner, "A Leptophobic Z' And Dark Matter From Grand Unification," Phys.Lett. B703 (2011) 343-347, arXiv:1106.3583 [hep-ph].

[62] J. M. Cline and A. R. Frey, "Light dark matter versus astrophysical constraints," Phys.Lett. B706 (2012) 384-388, arXiv:1109.4639 [hep-ph].

[63] K. Petraki, M. Trodden, and R. R. Volkas, "Visible and dark matter from a first-order phase transition in a baryon-symmetric universe," arXiv:1111.4786 [hep-ph].

[64] P. Gondolo, P. Ko, and Y. Omura, "Light dark matter in leptophobic Z' models," Phys.Rev. D85 (2012) 035022, arXiv:1106.0885 [hep-ph].

[65] J. Heeck and W. Rodejohann, "Kinetic and mass mixing with three abelian groups," Phys.Lett. B705 (2011) 369-374, arXiv:1109.1508 [hep-ph].

[66] K. S. Babu, C. F. Kolda, and J. March-Russell, "Implications of generalized $Z-Z$ ' mixing," Phys. Rev. D57 (1998) 6788-6792, arXiv: hep-ph/9710441. 
[67] E. J. Chun, J.-C. Park, and S. Scopel, "Dark matter and a new gauge boson through kinetic mixing," JHEP 02 (2011) 100, arXiv:1011.3300 [hep-ph].

[68] S. Cassel, D. Ghilencea, and G. Ross, "Electroweak and Dark Matter Constraints on a Z-prime in Models with a Hidden Valley," Nucl.Phys. B827 (2010) 256-280, arXiv:0903.1118 [hep-ph].

[69] A. Hook, E. Izaguirre, and J. G. Wacker, "Model Independent Bounds on Kinetic Mixing," Physical Review Letters (2010), arXiv:1006.0973 [hep-ph].

[70] Y. Mambrini, "The Kinetic dark-mixing in the light of CoGENT and XENON100," JCAP 1009 (2010) 022, arXiv:1006.3318 [hep-ph].

[71] CMS Collaboration, S. Chatrchyan et al., "Combined results of searches for the standard model Higgs boson in pp collisions at $\sqrt{s}=7 \mathrm{TeV}$," arXiv:1202.1488 [hep-ex].

[72] ATLAS Collaboration, "Combined search for the Standard Model Higgs boson using up to $4.9 \mathrm{fb}-1$ of pp collision data at $\sqrt{s}=7 \mathrm{TeV}$ with the ATLAS detector at the LHC," arXiv:1202.1408 [hep-ex].

[73] M. T. Frandsen, F. Kahlhoefer, A. Preston, S. Sarkar, and K. Schmidt-Hoberg, "LHC and Tevatron Bounds on the Dark Matter Direct Detection Cross-Section for Vector Mediators," JHEP 1207 (2012) 123, arXiv:1204.3839 [hep-ph].

[74] V. D. Barger, W.-Y. Keung and E. Ma, "A gauge model with light $W$ and $Z$ bosons," Phys. Rev. D 22, 727 (1980); M. Schmaltz and C. Spethmann, "Two simple $W^{\prime}$ models for the early LHC," JHEP 1107, 046 (2011) [arXiv:1011.5918].

[75] Q.-H. Cao, Z. Li, J.-H. Yu and C. P. Yuan, "Discovery and identification of $W^{\prime}$ and $Z^{\prime}$ in $S U(2) \times S U(2) \times U(1)$ models at the LHC," Phys. Rev. D 86, 095010 (2012) [arXiv:1205.3769].

[76] S. Dodelson, B. R. Greene and L. M. Widrow, "Baryogenesis, dark matter and the width of the Z", Nucl. Phys. B 372, 467 (1992); G. Belanger, B. Dumont, U. Ellwanger, J. F. Gunion and S. Kraml, "Global fit to Higgs signal strengths and couplings and implications for extended Higgs sectors," Phys. Rev. D 88, 075008 (2013) [arXiv:1306.2941].

[77] M. Cirelli, N. Fornengo and A. Strumia, "Minimal dark matter," Nucl. Phys. B 753, 178 (2006) [hep-ph/0512090].

[78] R. Barbieri, L. J. Hall and V. S. Rychkov, "Improved naturalness with a heavy Higgs: An alternative road to LHC physics," Phys. Rev. D 74, 015007 (2006) [hep-ph/0603188]. 
[79] A. Jinaru, C. Alexa, I. Caprini and A. Tudorache, poster presented at the International Conference on New Frontiers in Physics (Kolymbari, Creta), Sept. 2013. " $W^{\prime} \rightarrow h H^{ \pm}$decay in $G(221)$ models," J. Phys. G 41, 075001 (2014) [arXiv:1312.4268].

[80] Z. Sullivan, "Fully differential $W^{\prime}$ production and decay at next-to-leading order in QCD," Phys. Rev. D 66, 075011 (2002) [hep-ph/0207290].

[81] R. Gavin, Y. Li, F. Petriello and S. Quackenbush, "W Physics at the LHC with FEWZ 2.1," Comput. Phys. Commun. 184, 208 (2013) [arXiv:1201.5896 [hep-ph]].

[82] J. Alwall, M. Herquet, F. Maltoni, O. Mattelaer and T. Stelzer, "MadGraph 5 : Going Beyond," JHEP 1106, 128 (2011) [arXiv:1106.0522 [hep-ph]].

[83] A. Alloul, N. D. Christensen, C. Degrande, C. Duhr and B. Fuks, "FeynRules 2.0 - A complete toolbox for tree-level phenomenology," arXiv:1310.1921 [hep-ph]; C. Degrande, C. Duhr, B. Fuks, D. Grellscheid, O. Mattelaer and T. Reiter, "UFO - The Universal FeynRules Output," Comput. Phys. Commun. 183, 1201 (2012) [arXiv:1108.2040 [hep-ph]].

[84] J. Pumplin, D. R. Stump, J. Huston, H. L. Lai, P. M. Nadolsky and W. K. Tung, "New generation of parton distributions with uncertainties from global QCD analysis," JHEP 0207, 012 (2002) [hep-ph/0201195].

[85] G. Aad et al. [ATLAS Collaboration], "Search for dark matter in events with a hadronically decaying $W$ or $Z$ boson and missing transverse momentum in $p p$ collisions at $\sqrt{s}=8 \mathrm{TeV} "$, arXiv:1309.4017 [hep-ex].

[86] M. Beltran, D. Hooper, E. W. Kolb, Z. A. C. Krusberg and T. M. P. Tait, "Maverick dark matter at colliders," JHEP 1009, 037 (2010) [arXiv:1002.4137 [hep-ph]].

[87] Y. Bai, P. J. Fox and R. Harnik, "The Tevatron at the frontier of dark matter direct detection," JHEP 1012, 048 (2010) [arXiv:1005.3797];

[88] For a review, see A. Altheimer, S. Arora, L. Asquith, G. Brooijmans, J. Butterworth, M. Campanelli, B. Chapleau, A. E. Cholakian et al., "Jet Substructure at the Tevatron and LHC: New results, new tools, new benchmarks," J. Phys. G 39, 063001 (2012) [arXiv:1201.0008 [hep-ph]];

[89] Y. Bai and T. M. P. Tait, "Searches with mono-leptons," Phys. Lett. B 723, 384 (2013) [arXiv:1208.4361].

[90] CMS Collaboration, "Search for dark matter in the mono-lepton channel with $p p$ collision events at center-of-mass energy of 8 TeV", report CMS-PAS-EXO-13-004, July 2013. 
[91] T. Sjostrand, S. Mrenna and P. Z. Skands, "PYTHIA 6.4 physics and manual," JHEP 0605, 026 (2006) [hep-ph/0603175].

[92] J. Conway, R. Culbertson, R. Demina, B. Kilminster, M. Kruse, S. Mrenna, J. Nielsen, M. Roco, "Pretty Good Simulation of high energy collisions", http://physics.ucdavis.edu/ conway/research/software/pgs/pgs4-general.htm

[93] E. Conte, B. Fuks and G. Serret, "MadAnalysis 5, a user-friendly framework for collider phenomenology," Comput. Phys. Commun. 184, 222 (2013) [arXiv:1206.1599 [hep-ph]].

[94] L. M. Carpenter, A. Nelson, C. Shimmin, T. M. P. Tait and D. Whiteson, "Collider searches for dark matter in events with a $Z$ boson and missing energy," arXiv:1212.3352.

[95] G. D. Kribs, A. Martin and T. S. Roy, "Supersymmetry with a chargino NLSP and gravitino LSP," JHEP 0901, 023 (2009) [arXiv:0807.4936]; D. Curtin, P. Jaiswal and P. Meade, "Charginos hiding in plain sight," Phys. Rev. D 87, no. 3, 031701 (2013) [arXiv:1206.6888].

[96] CMS Collaboration, "Search for electroweak production of charginos, neutralinos, and sleptons using leptonic final states in $p p$ collisions at $8 \mathrm{TeV}$ ", report CMSPAS-SUS-13-006, July 2013; ATLAS Collaboration, "Search for direct production of charginos and neutralinos in events with three leptons and missing transverse momentum in $21 \mathrm{fb}^{-1}$ of pp collisions at $\sqrt{s}=8 \mathrm{TeV}$ ", report ATLAS-CONF2013-035, April 2013.

[97] G. Aad et al. [ATLAS Collaboration], Phys. Lett. B 716, 1 (2012) [arXiv:1207.7214 [hep-ex]].

[98] S. Chatrchyan et al. [CMS Collaboration], Phys. Lett. B 716, 30 (2012) [arXiv:1207.7235 [hep-ex]].

[99] The ATLAS collaboration, ATLAS-CONF-2014-009.

[100] [CMS Collaboration], CMS-PAS-HIG-13-005.

[101] G. Aad et al. [ATLAS Collaboration], Phys. Lett. B 726, 88 (2013) [arXiv:1307.1427 [hep-ex]].

[102] S. Dawson, A. Gritsan, H. Logan, J. Qian, C. Tully, R. Van Kooten, A. Ajaib and A. Anastassov et al., arXiv:1310.8361 [hep-ex].

[103] J. F. Gunion, H. E. Haber, G. L. Kane and S. Dawson, Front. Phys. 80, 1 (2000); J. F. Gunion, H. E. Haber, G. L. Kane and S. Dawson, hep-ph/9302272. 
[104] J. F. Gunion and H. E. Haber, Phys. Rev. D 67, 075019 (2003) [hep-ph/0207010].

[105] U. Baur, T. Plehn and D. L. Rainwater, Phys. Rev. Lett. 89, 151801 (2002) [hep$\mathrm{ph} / 0206024]$.

[106] U. Baur, T. Plehn and D. L. Rainwater, Phys. Rev. D 67, 033003 (2003) [hep$\mathrm{ph} / 0211224]$.

[107] U. Baur, T. Plehn and D. L. Rainwater, Phys. Rev. D 68, 033001 (2003) [hepph/0304015].

[108] U. Baur, T. Plehn and D. L. Rainwater, Phys. Rev. D 69, 053004 (2004) [hep$\mathrm{ph} / 0310056]$.

[109] T. D. Lee, Phys. Rev. D 8, 1226 (1973).

[110] P. Fayet, Nucl. Phys. B 78, 14 (1974).

[111] R. D. Peccei and H. R. Quinn, Phys. Rev. Lett. 38, 1440 (1977).

[112] P. Fayet and S. Ferrara, Phys. Rept. 32, 249 (1977).

[113] M. S. Carena and H. E. Haber, Prog. Part. Nucl. Phys. 50, 63 (2003) [hepph/0208209].

[114] R. Contino, M. Ghezzi, M. Moretti, G. Panico, F. Piccinini and A. Wulzer, JHEP 1208, 154 (2012) [arXiv:1205.5444 [hep-ph]].

[115] C. -R. Chen and I. Low, arXiv:1405.7040 [hep-ph].

[116] C. O. Dib, R. Rosenfeld and A. Zerwekh, JHEP 0605, 074 (2006) [hepph/0509179].

[117] L. Wang, W. Wang, J. M. Yang and H. Zhang, Phys. Rev. D 76, 017702 (2007) [arXiv:0705.3392 [hep-ph]].

[118] L. Wang and J. M. Yang, Phys. Rev. D 77, 015020 (2008) [arXiv:0710.5038 [hepph]].

[119] X. -F. Han, L. Wang and J. M. Yang, Nucl. Phys. B 825, 222 (2010) [arXiv:0908.1827 [hep-ph]].

[120] M. Gillioz, R. Grober, C. Grojean, M. Muhlleitner and E. Salvioni, JHEP 1210, 004 (2012) [arXiv:1206.7120 [hep-ph]].

[121] R. Grober and M. Muhlleitner, JHEP 1106, 020 (2011) [arXiv:1012.1562 [hep-ph]]. 
[122] E. Asakawa, D. Harada, S. Kanemura, Y. Okada and K. Tsumura, Phys. Rev. D 82, 115002 (2010) [arXiv:1009.4670 [hep-ph]].

[123] S. Dawson, C. Kao and Y. Wang, Phys. Rev. D 77, 113005 (2008) [arXiv:0710.4331 [hep-ph]].

[124] S. Dawson, E. Furlan and I. Lewis, Phys. Rev. D 87, 014007 (2013) [arXiv:1210.6663 [hep-ph]].

[125] G. D. Kribs and A. Martin, Phys. Rev. D 86, 095023 (2012) [arXiv:1207.4496 [hep-ph]].

[126] M. J. Dolan, C. Englert and M. Spannowsky, JHEP 1210, 112 (2012) [arXiv:1206.5001 [hep-ph]].

[127] M. J. Dolan, C. Englert and M. Spannowsky, Phys. Rev. D 87, no. 5, 055002 (2013) [arXiv:1210.8166 [hep-ph]].

[128] J. Liu, X. -P. Wang and S. -h. Zhu, arXiv:1310.3634 [hep-ph].

[129] J. Cao, Z. Heng, L. Shang, P. Wan and J. M. Yang, JHEP 1304, 134 (2013) [arXiv:1301.6437 [hep-ph]].

[130] U. Ellwanger, JHEP 1308, 077 (2013) [arXiv:1306.5541, arXiv:1306.5541 [hep$\mathrm{ph}]$.

[131] C. Han, X. Ji, L. Wu, P. Wu and J. M. Yang, JHEP 1404, 003 (2014) [arXiv:1307.3790 [hep-ph]].

[132] A. Arhrib, hep-ph/0012353.

[133] M. Maniatis, A. von Manteuffel, O. Nachtmann and F. Nagel, Eur. Phys. J. C 48, 805 (2006) [hep-ph/0605184].

[134] V. Barger, L. L. Everett, H. E. Logan and G. Shaughnessy, Phys. Rev. D 88, 115003 (2013) [arXiv:1308.0052 [hep-ph]].

[135] C. -Y. Chen, S. Dawson and M. Sher, Phys. Rev. D 88, 015018 (2013) [arXiv:1305.1624 [hep-ph]].

[136] N. Craig, J. Galloway and S. Thomas, arXiv:1305.2424 [hep-ph].

[137] K. Cheung, J. S. Lee and P. -Y. Tseng, JHEP 1401, 085 (2014) [arXiv:1310.3937 [hep-ph]].

[138] B. Dumont, J. F. Gunion, Y. Jiang and S. Kraml, arXiv:1405.3584 [hep-ph].

[139] H. E. Haber, G. L. Kane and T. Sterling, Nucl. Phys. B 161, 493 (1979). 
[140] R. M. Barnett, G. Senjanovic, L. Wolfenstein and D. Wyler, Phys. Lett. B 136, 191 (1984).

[141] R. M. Barnett, G. Senjanovic and D. Wyler, Phys. Rev. D 30, 1529 (1984).

[142] Y. Grossman, Nucl. Phys. B 426, 355 (1994) [hep-ph/9401311].

[143] A. Pich and P. Tuzon, Phys. Rev. D 80, 091702 (2009) [arXiv:0908.1554 [hep-ph]].

[144] G. Cree and H. E. Logan, Phys. Rev. D 84, 055021 (2011) [arXiv:1106.4039 [hepph]].

[145] W. Altmannshofer, S. Gori and G. D. Kribs, Phys. Rev. D 86, 115009 (2012) [arXiv:1210.2465 [hep-ph]].

[146] Y. Bai, V. Barger, L. L. Everett and G. Shaughnessy, Phys. Rev. D 87, no. 11, 115013 (2013) [arXiv:1210.4922 [hep-ph]].

[147] W. Yao, arXiv:1308.6302 [hep-ph].

[148] V. Barger, L. L. Everett, C. B. Jackson and G. Shaughnessy, Phys. Lett. B 728, 433 (2014) [arXiv:1311.2931 [hep-ph]].

[149] CMS Collaboration [CMS Collaboration], CMS-PAS-HIG-13-025.

[150] CMS Collaboration [CMS Collaboration], CMS-PAS-HIG-13-032.

[151] G. Aad et al. [ATLAS Collaboration], arXiv:1406.5053 [hep-ex].

[152] M. Carena, I. Low, N. R. Shah and C. E. M. Wagner, JHEP 1404, 015 (2014) [arXiv:1310.2248 [hep-ph]].

[153] T. Plehn, M. Spira and P. M. Zerwas, Nucl. Phys. B 479, 46 (1996) [Erratum-ibid. B 531, 655 (1998)] [hep-ph/9603205].

[154] O. J. P. Eboli, G. C. Marques, S. F. Novaes and A. A. Natale, Phys. Lett. B 197, 269 (1987).

[155] E. W. N. Glover and J. J. van der Bij, Nucl. Phys. B 309, 282 (1988).

[156] D. A. Dicus, C. Kao and S. S. D. Willenbrock, Phys. Lett. B 203, 457 (1988).

[157] B. Hespel, D. Lopez-Val and E. Vryonidou, arXiv:1407.0281 [hep-ph].

[158] S. Dawson, S. Dittmaier and M. Spira, Phys. Rev. D 58, 115012 (1998)

[159] S. Dittmaier et al. arXiv:1101.0593 [hep-ph].

[160] D. Y. Shao, C. S. Li, H. T. Li and J. Wang, arXiv:1301.1245 [hep-ph]. 
[161] D. de Florian and J. Mazzitelli, Phys. Lett. B 724, 306 (2013)

[162] F. Goertz, A. Papaefstathiou, L. L. Yang and J. Zurita, JHEP 1306, 016 (2013)

[163] D. de Florian and J. Mazzitelli, Phys. Rev. Lett. 111, 201801 (2013) [Phys. Rev. Lett. 111, 201801 (2013)] [arXiv:1309.6594 [hep-ph]].

[164] V. Barger, L. L. Everett, C. B. Jackson, A. Peterson and G. Shaughnessy, arXiv:1408.0003 [hep-ph].

[165] M. Moretti, S. Moretti, F. Piccinini, R. Pittau and A. D. Polosa, JHEP 0502, 024 (2005) [hep-ph/0410334].

[166] A. Arhrib, R. Benbrik, C. -H. Chen, R. Guedes and R. Santos, JHEP 0908, 035 (2009) [arXiv:0906.0387 [hep-ph]].

[167] B. Bhattacherjee and A. Choudhury, arXiv:1407.6866 [hep-ph].

[168] J. Baglio, O. Eberhardt, U. Nierste and M. Wiebusch, Phys. Rev. D 90, 015008 (2014) [arXiv:1403.1264 [hep-ph]].

[169] J. Baglio, A. Djouadi, R. Groöber, M. M. Mühlleitner, J. Quevillon and M. Spira, JHEP 1304, 151 (2013) [arXiv:1212.5581 [hep-ph]].

[170] D. E. Ferreira de Lima, A. Papaefstathiou and M. Spannowsky, arXiv:1404.7139 [hep-ph].

[171] N. Chen, C. Du, Y. Fang and L. -C. Lu, Phys. Rev. D 89, 115006 (2014) [arXiv:1312.7212 [hep-ph]].

[172] G. Aad et al. [ATLAS Collaboration], arXiv:0901.0512 [hep-ex].

[173] ATLAS Collaboration, ATL-PHYS-PUB-2013-004

[174] H. Baer, V. Barger, G. Shaughnessy, H. Summy and L. -t. Wang, Phys. Rev. D 75, 095010 (2007) [hep-ph/0703289 [HEP-PH]].

[175] R. J. Barlow, J. Comput. Phys. 72, 202 (1987).

[176] V. M. Abazov et al. [D0 Collaboration], Phys. Rev. D 78, 012005 (2008) [arXiv:0803.0739 [hep-ex]].

[177] V. Bartsch and G. Quast, CERN-CMS-NOTE-2005-004.

[178] G. Aad et al. [ATLAS Collaboration], JHEP 1301, 086 (2013) [arXiv:1211.1913 [hep-ex]].

[179] A. Djouadi, J. Kalinowski and M. Spira, Comput. Phys. Commun. 108, 56 (1998) [hep-ph/9704448]. 